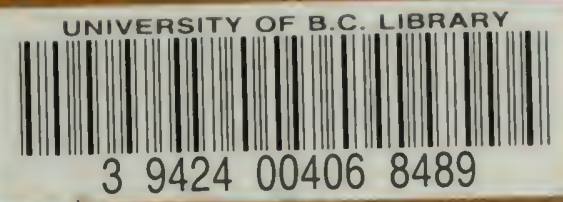

STORAGE ITEM PRUCESS ING-ONE

-PI-A 150

U.B.C. LIBRARY 


\section{THE LIBRARY}

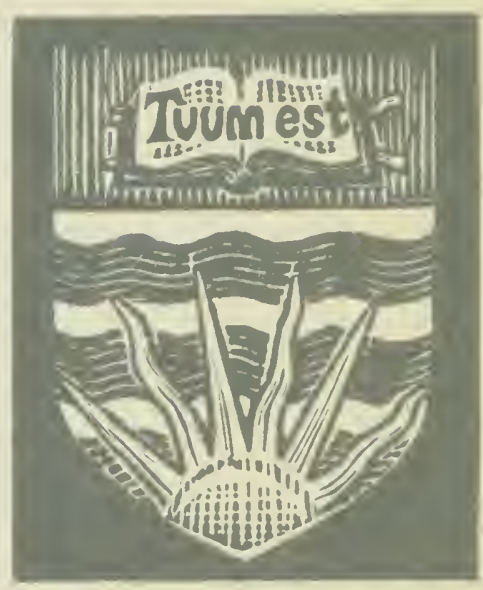

THE UNIVERSITY OF BRITISH COLUMBIA 

Digitized by the Internet Archive in 2010 with funding from University of British Columbia Library 


LES MOUVEMENTS

DES VÉGETAUX 


\section{COLLECTION}

\section{" LES MAITRES DE LA PENSÉE SGIENTIFIQUE"}

\section{Viennent de paraitre :}

HuxGHeNs (Christian). - Traité de la Lumiere. Un vol. in- 16 double couronne $(180 \times 115)$ de $x u 1-156$ pages; 1920; broché, net................................................

LAVOISIER. - Mémoires sur la respiration et la transpiration des animaux....................................

SPALlanzaNI (Lazare). - Observations et Expériences jaites sur les Animalcules des Infusions. Tome Ier. Un vol. in-16 double couronne $(180 \times 115)$ de viII106 pages; 1920; broché, net.

- Tome II. Un vol. in-16 double couronne $(180 \times 115)$ de 122 pages; 1920 ; broché, net................................

ClaIraut. - Eléments de Géométrie. 2 vol. brochés; chaque volume broché, net.

LAVOISIER et LAPLACE. - Mémoire sur la Chaleur....

CARNot. - Réflexions sur la métaphysique du Calcut infinitésimal. 2 vol. brochés.

D'ALEMBERT. - Traité de Dynamique.

DuTrochet, Les mouvements des végétaux. - Du réveil et du sommeil des plantes.

\section{Sous presse :}

AMPÈR, De l'action exercée sur un courant électrique par un autre courant, etc., avec la lettre à Van Beck.

LAPLACE, Essai philosophique sur les probabilites.

\section{Paraitront prochainement :}

HERTz, Equations électrodynamiques fondamentales des corps en-mouvement et des corps en. repos.

GALILEE, Dialogues et démonstrations concernant deux sciences nouvelles.

Bouguer, Essai d'optique sur la gradation de la lumière.

NEWTON, Principes malhématiques de la philosophie naturelle. Eic., etc.

$$
\text { DEMANDER LE PROSPECTUS SPÉCIAL }
$$

Il est tiré de chaque volume 10 exemplaires sur papier do Hollande, au prix uniforme et net de 6 francs. 
LES MATTES DE LA PENSÉE SGIENTIRIQUE Colleotion de Mémolres et OUVRaGes

Publiee par les soins de Maurice SOLOVINE

\section{L.ES MOUVEMENTS DES VEGETAUX}

DU REVELL

ET DU

SOMMEIL DES PLANTES

PAR

René DUTROGHET

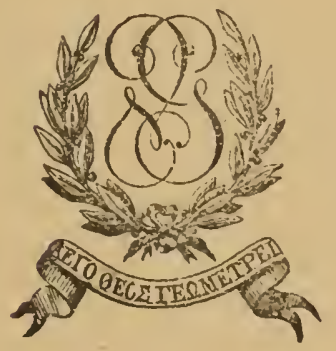

PARIS

GAUTHIER-VILLARS ET Cie, ÉDITEURS

LIBRAIRES HU BUREAU DES LONGITEDES, DE L'ECOLE POLYTECLLNIQUE Quai des Grands-Augustins, 55 
MAC.

QR 771

D 88

1921

$-1$ 


\section{AVERTISSEMENT}

L'accroissement rapide des découvertes scien. tifiques engendre falalcment l'oubli des découvertes passées et de leurs auteurs - oubli encore favorisé par le fait regrettable que la plupart des mémoires et des ourrages, oì ces découvertes se trouvent exposées, sont complètement épuisés et introuvables.

La collection des Maitres de la Pensée scientifique comprendra les mémoires et les ouvrages les plus importants de tous les temps et de tous les pays. Elle est destinéc à rendre accessibles aur sarants et au public cultivé les travaux originaux, qui marquent les étapes successives dans la construction lcnte et laborieuse de l'édifice scientifique. Tous les domaines de la Science y seront représentés: les mathématiques, l'astronomie, la physique, la chimic, la géologie, les sciences naturelles et biologiques, la méthodologie et la philosophie des sciences. Etant la plus complète, elle fournira les documents indispensables aux historiens de la science et de la civilisation, qui voudront étudier l'évolution de l'esprit humain sous sa forme la plus élevée. Elle permettra aux savants de connaître plus intimement les décou- 
vertes de leurs devanciers et d'y trouver nombre d'idées originales. Les philosophes y trouveront une mine inépuisable pour l'étude épistémologique des théories, des hypothèses et des concepts, au moyen desquels se construit la connaissance de l'univers. Elle offrira enfin à la jeunesse studieuse un moyen facile et peu cô̂teux de prendre contact à leur source même avec les méthodes expérimentales et les procédés ingénieux que les grands chercheurs ont dî inventer pour résoudre les diffcultés - méthodes concrètes, infiniment plus suggestives et plus fécondes que ne sont les règles schématiques des Manuels.

On trunive encore dans les nérnoires classiques, où la profondeur de la pensée et la justesse du raisonnernent se manifestent sous une forme remarquablement lucide et élégante, le secret d'exposer les découvertes scientifiques d'une façon claire et précise, comme l'ont demandé à plusieurs reprises les savants les plus illustres de notre temps.

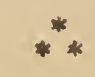

Les mémoires et les ouvrages français seront réimprimés arec grande exactitude d'après les textes originaux les mieux établis, et ceux des savants étrangers seront traduits intégralement et avec une rigoureuse fidélité. 


\section{Notice biographique.}

liené-Joachim-Henri Durrochet naquit au château de Néon Indre) le 14 novembre 1776, et mourut à Paris le 4 février 1847. Son père ayant émigré au moment où la Révolution éclata, tous ses biens furent confisqués, et le jeune Dutrochet se trouva ainsi complètement privé de ressources et obligé de gagner son 2xistence. En 17\$9, il postula un poste dans la marine, mais à peine l'avait-il obtenu qu'il changea d'avis avant même de :embar'quer.

En 1802, il commença à Paris l'étude de la médecine et fut reçu docteur en 1806. Ayant été promu médecin militaire en ISos, il accompagna en cette qualité le roi Joseph Bonaparte en Espagne. A l'hôpital militaire de Burgos, dont il était le lirecteur, il ent à combattre une épidémie typhoïde des plus meurtric̀res. Atteint lui-me̊me, il guérit très lentement et fut tellement affaibli qu'il se vit oblfgé ce se démettre de ses fonctions. Il rentra en France en 1809 et se flxa dans une maison de campagne aux environs cle Châteaurenault, en Touraine, où vivait sa mère.

A partir de cette époque Dutrochet se consacra entièrement i l'étnde des sciences naturelles, et plus particulièrement à celle de l'embryogénie animale et de la physiologie végétale, ù, par ses nombreuses et délicates expériences, il a fait des lécouvertes fondamentales, qui ont eu une influence considérable sur le développement de ces branches de la biologie. Il est le premier à avoir tenté d'expliquer les mouvements de la sève à l'intérieur des plantes par les phénomènes de la diosmose, et à avoir étudié parallèlement l'absorption de l'oxygène par les plantes et par les animaux. Les phénomènes qu'il étudia en outre avec une prédilection marquée sont ceux de l'endosmose. Il s'est efforcé de mettre en lumière son action mécanique et l'extrême importance qu'elle présente pour l'explication de certaines fonctions végétales. C'est cette théorie qui lui a nermis de ramerier à des principes de mécaniçue des phénomènes botaniques qu'on s'est contenté d'expliquer avant 
lui par la force vitale. C'est grãce à slle surtout qu'll est arrivé à donner une explication satisfaisante des mouvements de la sève montante et descendante.

Adversaire irréductible du vitalisme, il s'est donné comme tâche d'expliquer tous les mouvements et toutes les fonctions des plantes et des êtres animés par des lols purement physiques. " L'admission d'une différence essentielle et fondamentale entre les lois physiques et les lois psychologiques m'a toujours paru contraire à une saine philosophie. Les êtres vivants doivent être considérés comme des laboratoires dans lesquels la nature opère des phénomènes et confectionne des substances qui" ne peuvent avoir de durée que sous l'intluence des callses particulières qui ont présidé à leur prolluction. La vie se compose de phénomènes physiques et chimiques spéciaux qui doivent se rattacher à la physique et à la chimie généralc. Il faut donc chercher à découvrir quels sont les phénomènes spéciaux de la physique et de la chimie auxquels le mouvement vital doit son existence. Je pense avoir fait le premier pas dans cette voie par la découverte de l'endosmose. " (Note inédite écrite en 1835).

Ces admirables idées sont précisẻment celles qui ont guidé dans leurs recherches tous les grands biologistes qui sont venus après lui, et il ne faudra jamais les perdre de vue si l'on veut réaliser des progrès réels et durables en biologie.

Dutrochet a réuni en 1837 ses travaux les plus importants dans deux volumes qui portent le titre suivant: Mémoires pour servir à l'histoire anatomique et physiologique des végétaux et des animaux. C'est d'après cette édition que nous reproduisons les denx mémoires figurant dans ce volume. Outre ces mémoires, ses œuvres contiennent encore des recherches remarquables sur l'embryogènie animale, sur la physiologie végétale, sur les rotifères, sur la métamorphose du canal alimentalre chez les insectes, sur la structure et la régénération des plumes, sur l'ostéogénie, etc. Son travail de tout premier ordre sur l'Endosmose sera reproduit prochainement dans cette Collection.

M. S. 


\section{IOUVEMENTS DES VŔGETAUX}

COUP-D'CEIL GENÉRAL

SUR

\section{LES MOUVEMENTS DES VÉGETAUX}

\section{§ I.- ExaMen du MÉCANisme}

DES MODES ǴLÉMENTAIRES DE MOUVEMENT

PAR INCURVATION ET PAR TORSION ${ }^{(1)}$.

La faculté do se mouvoir, si libéralement accordée ar la nature aux animaux, n'a point à beaucoup rès été refusée aux végétaux. Dans une foule d'ocasions, ils meuvent spontanément quelques-unes de eurs parties, soit pour leur donner une position u une direction convenable à l'exercice de leurs onctions, soit pour obéir ì une influence de nature nconnue qu'exercent sur eux-les causes excitantes. Iais ce n'est pas toujours ì l'occasion de l'influence 'une cause extérieure, que les végétaux meuvent uelques-unos de leurs parties. Il y a, en effet, chez ux, des mouvements dus à une sorte d'élasticité, aquelle diffère de l'élasticité des substances miné-

(1) Le paragraphe II de ce mémoire a été publié en 1828; tont reste parait ici pour la première fois. 
rales en cela que, dépendant de la présence de l'ear dans le tissu végétal, elle disparaît avec ce liquidı lorsqu'il cesse de remplir les cellules végétales. $J_{1}$ ferai voir ailleurs, que l'eau n'est pas la seule subs tance dont l'introduction dans le tissu végétal soi propre à donner à ce tissu une tendance à se courbes élastiquement, et dans d'autres circonstances unt tendance à se tordre sur lui-même. Ainsi, il peuı apparaître et disparaître dans les tissus végétaux deux tendances au mouvement, la première par incurvation et la seconde par torsion. Ces deux modes de mouvement sont ceux que je nomme modes élémentaires. Ce sont eux qui président à tous les mouvements des végétaux.

Les mouvements que les végétaux exécutent, considérés sous lo rapport des circonstances dans les. quelles ils ont lieu, et sous le rapport des phé. nomènes auxquels ils coopèrent, peuvent être rapportés à cinq divisions :

$1^{\circ}$ Les mouvements élémentaires d'incurvation ef de torsion.

$2^{\circ}$ Les mouvements particuliers par lesquels les fleurs ou les feuilles de certains végétaux pren. nent les positions successives qui constituent ce que l'on a nommé le sommeil et le réveil.

$3^{\circ}$ Les mouvements d'incurvation par excitation autrement dits mouvements d'irritabilité.

$4^{\circ}$ Les mouvements par lesquels les végétaux diri gent les radicules de leurs embryons séminaux dan: - le sens de la pesanteur, et leurs tiges dans le sen: opposé à celui de cette même pesanteur. 
$5^{\circ}$ Les mouvements par lesquels les végétaux dirient quelques-unes de leurs parties vers la lumière, u- bien dans le sens opposé à celui de son afflux.

L'ordre numórique dans lequel je place ici les zouvements végétaux paraîtra peu naturel et arbiraire au premier coup-d'œil, mais on verra qu'il ésulte nécessairement do l'enchaînement des faits. Yhacune de ces divisions des mouvements végétaux era l'objet d'un mémoire séparé.

Les mouvements élémentaires d'incurvation et de orsion scront seuls étudiés spécialement dans co aémoire. Les mouvements d'incurvation élémentaire ésultent d'une tendance à la courbure dans un ens déterminé, tendance à laquelle est opposé un bstacle. Ce dernier venant à être vaincu, le mou'ement d'élasticité qui est la conséquence de cette endance s'exécute librement, et il s'arrête lorsqu'il st accompli. Les exemples en sont nombreux dans e règne végétal. Les uns appartiennent à l'état de ie, les autres n'ont lieu que dans certaines parties régétales qui ont cessé de vivre. Je vais étudier le nécanisme de ces mouvements chez un petit nombre lo végiétaux, choisis parmi ceux qui sont à la fois t les plus vulgaires et les plus faciles à étudier ous ce point de vue.

\section{§ II. - MouveMents PAR INCURVATION.}

Mrécanisme du mouvement dans les valves du pérlcarpe de la balsamine (impatiens balsamina).

On sait que les valves du péricarpe de la balsa-nine, à l'époque de la maturité, se séparent les unes 
des autres, et que chacune d'elles se roule en spiral en dedans, c'est-à-dire que sa convexité est er dehors, ou du côté de l'épiderme. Si on les redrasse elles retournent spontanément et avec vivacité leur ćtat d'incurvation, lorsqu'on les abandonne ¿̀ clles-mêmes. Si on les plonge dans l'eau, elles st courbent encore plus profondément; si on les laisse se dessécher à moitié, elles tombent dans l'état de flaccidité ou do relâchement, et perdent leur ten dance élastique à l'incurvation. Ces premiers fait prouvent déjà que la présence de l'eau dans les organes qui composent le tissu de la valve est une des conditions de l'existence de sa tendance à l'in curvation. Si l'on plonge dans l'eau la valve ̀̀ moitié flétrie par l'évaporation de ses liquider intérieurs, elle absorbe ce liquide, reprend son éta1 turgide vital et son incurvation élastique. Si or laisse dessécher presque entièrement la valve à l'air libre, ello ne reprend plus du tout son état turgide et son incurvation lorsqu'on la plonge dans l'eau. Elle s'imbibe entièrement, et jusqu'à complète saturation, mais elle n'absorbe point l'eau ave excès comme elle lo faisait auparavant; ello ne redevient point turgide; elle demeure constamment dans l'état de flaccidité. Cette dernière expérience m'a condüit à penser que l'incurvabilité tenait à l'existence du liquide organique qui remplissait les organes collulaires dont la valve est composée, et que c'était, non par une simple imbibicion, mais par endosinose, que l'eau était introduite dans le tissu organique incurvable. Les expériences qu: ront être exposées confirmeront ce premier aperçu. 
Le tissu organique qui compose la valve du péricarpe de la balsamine, vu au microscope, se trouve composé par une agrégation d'utricules ou de cellules. Ces cellules, grandes à la partie externe, vont toujours en décroissant de grosseur, jusqu'à la pritie interne, où elles sont le plus petites. Cette disposition dévoile la cause de la tendance à l'incurvation. Toutes les cellules étant pleines jusqu'à l'état turgide, l'incurvation de la valve en dedans en ost le résultat nécessairc. Les cellules qui composent ce tissu sont, dans l'état naturel, remplies par un liquide organique plus ou moins dense. Lorsque ces cellules 'éprouvent extérieurement l'accession de l'eau, elles exeroent l'endosmose implétive, par cela seul qu'elles contiennent un liquide organique plus dense que l'eau. Alors elles deviennent turgides, et le tissu, distendu plus en dehors qu'en dedans, prend un état d'incurvation en dedans (1). Lorsqu'une dessication prolongée a enlevé le liquide intézieur des cellules, celles-ci s'imbibent do l'eau dont elles éprouvent extérieurement l'aceession, mais elles n'ereicent pius d'endosmose implétive; elles ne deviennent plus turgides; le tissu demeure dans l'état de fiaccidité; l'incurvabilité est abolic. Du moment qu'il mo fut démontré que l'accession extérieure de l'eau était la cause de l'endosmose implétive des cellules qui contenaient un liquide organique dənse, et que cette endosmose

(1) Toutes les fois que je dirai, en parlant d'une partie régétale, qu'elle se courbe en dedurs ou qu'elle se courbe en dehors, cela signifiera, dans le premier cas, qlie la concavité de la courbure est tournée vers l'intérieur on le centre du végétal, et, dans le second cas, que la coucavité de la courbure est tournce vers l'extérieur. 
était la cause de l'état turgide du tissu; du moment qu'en outre il me fut démontré que l'incurvation do ce tissu était le résultat de l'inégalité de ses cellules, grandes en dehors, et petites en dedans, il me parut certain qu'en substituan' à l'eau un liquide plus denso quo celui que contenaient les cellules, je produirais, non plus l'endosmose implétive, mais l'endosmose déplétive (1) et, par suite, une incurvation de la valve dans le sens opposé à celui de son incurvation naturelle. Je plongeai donc plusieurs de ces valves, qui étaient courbées en dedans, dans du sirop do sucre. Elles ne tarderent pas ì perdre leur état d'incurvation et à devenir droites. Bientôt après, elles se roulèrent en spirale en dehor's. Cet effet, que j'avais prévu, était un résultat nécessaire de l'endosmose déplétive, qui soutirait le liquide organique moins dense que le sirop liquide qui emplissait les cellules du tissu de la valve. Ces cellules étant désemplies, la valve se roulait en dehors, parce que, de ce côté, les cellules, plus grandes, avaient plus perdu de liquide; il y avait, de ce côté, moins de matière solide qu'en dedans; dès lors, il devait y avoir incurvation de ce côté, lors de la soustraction d'une grande partie du liquide, qui, en gonflant ces cellules, leur faisait occuper un espace considérable. Jo transportai dans l'eau ces valves roulées en spirale en dehors; elles ne tardèrent pas à se dérouler, et, enfin, à reprendre leur état naturel d'incurvation en dedans; ici, leurs

(1) Pour l'intelligence de ce que j'entends par endosmose implétive et endosmose déplétive, voyez dans le premier Mémoire [De l'endosmose, in Mémoires, t. I. Paris, 1837], aux rages 10, 11 et 14. 
cellules composantes exerçaiont de nouveau l'endosmose implétive, et l'incurvation en dedans en était lo résultat. Je transportai de nouveau mes valves dans le sirop. Elles se roulèrent en dehors; jo les replaçai dans l'eau, elles se courbèrent en dedans. Je r'épétai ce double jeu d'incurvation neuf fois en einq heures de temps. Alors les valves cessèrent de se courber en dedans, lorsque je les plongeais dans l'eau; elles ne reprenaient plus assez pour cela leur état turgide, ce qui provenait de ce que l'action d'endosmose déplétive, provoquée par l'immersion dans le sirop, avait soutiré en grande partie leur liquide dense intérieur; il ne leur en restait plus assez pour exercer une endosmose implétive suffisante pour les replacer dans l'état turgide; dès lors, il n'y avait plus d'incurvation en dedans. Mais l'immersion dans le sirop produisait toujour's lo roulement en dehors, jusqu'au summum, parce que cette incurvation était le résultat do l'endosmoso déplétive, laquelle, loin d'éprouver do la diminution, allait, au contraire, toujours en augmentant d'ćnergie, puisque le liquido intérieur des cellules devenait de moins en moins dense, l'eau ayant remplacé huit ou neuf fois le liquide organique intérieur, soutiré par l'endosmose déplétive qu'occasionnait l'immersion dani le sirop. Je mis sous lo microscope uno lame minco de valve, plongée dans du sirop de sucre; je fus ainsi à mêmo de voir d'une manière immédiate le mécanisme de son incurvation. Je vis toutes les cellules, et spéciale ment les plus grandes, qui occupaient son côté extérieur convexe, perdre assez rapidement de leur 
diamètre, par l'effet de leur déplétion, et l'incurvation en dehors de la lame de valve en fut l'effet.

Il résulte de ces expériences, que les valves du péricarpe de la balsamine pordent leur incurvabilité ou leur faculté d'incurvation élastique en dedans, lorsque le liquide organique dense qui remplit leurs cellules est soutiré, soit par l'évaporation, soit par l'endosmose déplétive. C'est done à l'existence de ce liquide intérieur dense qu'est due l'incurvabilité. Si l'on pouvait rendre aux cellules le liquide dense $q u$ 'elles ont perdu, on leur rendrait leur faculté de devenir turgides par endosmose implétive, lors do l'accession extérieure de l'eau : on rendrait par conséquent aux valves leur faculté de prendre une incurvation en dedans. C'est effectivement ce que j'ai fait par les deux expériences suivantes. J'ai fait dessécher à l'air libre des valves de péricarpe de balsamine, en avant soin de les empêcher de se tortiller, et de les conserver dans la rectitude. Lorsque cette dessication me parut à peu près complète, j'achevai de la déterminer à l'aide de la chaleur douce du feu. Les ralves ainsi desséchées étaient devenues cassantes et friables. J'en plongeai quelques-unes dans l'eau; elles s'imbibèrent jusqu'à saturation, et demeurèrent droites dans l'état de flaccidité. Je plongeai plusieurs autres de ces valves dans de l'eau très sucrée; elles s'imbibèrent de ce liquide dense juscqu'ì saturation, et demeurèrent de même dans l'état de rectitude et de flaccidité. Lorsque je jugeai quo les cellules composantes de leur tissu avaient absorbé par imbibition du liquide sucré autant qu'elles pouvaient le faire, en vertu 
de leur simple capillarité, je plongeai ces valves dans l'eau; elles ne tardèrent pas à l'absorber par' l'effet de l'endosmose implétive, provoquée par la présence d'un liquide dense dans les cellules; leur tissu cellulaire devint turgide, et l'incurvation des valves en dedans eut lieu de la même manière que dans l'état naturel. Je transportai ces valves dans du sirop de sucre, elles se roulèrent en dehors; je les replaçai dans l'eau, elles se courbèrent de nouveau en dedans; en un mot, ces valves avaient repris leur incurvabilité par une sorte de résurrection; seulement leur incurvation n'avait pas autant de force d'élasticité que dans l'état naturel.

$\mathrm{Je}$ viens d'exposer comment l'immersion alternative, souvent répétée dans le sirop et dans l'eau, avait fini par soutirer la plus grande partie du liquide organique dense que contenaient originairement les cellules, en le remplaçant par de l'eau. Il résultait de là l'impossibilité au tissu do la valve de reprendre dorénavant son état turgide, et par conséquent son incurvation en dedans; mais, en abandonnant longtemps dans le sirop ces valves ainsi privées do leur liquide dense naturel, ce liquide sucré tend à les pénétrer par imbibition. Les cellules s'en remplissent, en sorte qu'au bout de. huit à dix jours, si l'on transporto ces valves dans l'eau, elles quittent leur incurvation en dehors, et reprennent leur incurvation naturelle en dedans; elles ont récupéré leur incurvabilité en récupérant un liquide dense dans l'intérieur de leurs cellules.

Il résulte de ces observations, que les valves du péricarpe de la balsamine possèdent une faculté 
d'incurvation élastique qui résulte de l'état turgide par endosmose implétive d'un tissu cellulaire à cellules larges et rares au côté convexe, petites et serrées au côté concave. C'est l'accession extérieure do l'eau sur ces cellules remplies d'un liquide organique dense, qui détermine l'endosmose implétive de ces cellules, et par conséquent l'exercice de l'incurvabilité, dont le mécanisme se trouve ainsi dévoilé. Dans l'état naturel, e'est la sève lymphatique ascendante, qui n'est presque que de l'cau pure, qui remplit ici le rôle de liquide extérieur, dont l'accession provoque l'endosmose implétive des cellules. On peut se convaincre de cette vérité, en laissant flétrir un rameau de balsamine détaché de la plante et chargé de fruits. En perdant une partie de l'eau qui les rend turgides, les valves des péricarpes perdent une partie de leur incurvabilité; elles la récupèrent en plongeant l'extrémité du rameau dans l'eau. Ce liquide, pompé par la tige, arrive par les canaux lymphatiques jusqu'aux cellules des valves, et son accession extérieure détermine leur endosmose implétive, et par conséquent le retour de leur état turgide, ce qui ramène leur incurvabilité.

Il était important d'apprécier l'action des différents agents chimiques sur l'incurvabilité végétale. $J$ e me suis assuré que les acides affaiblis augmentaient la force de la tendance à l'incurvation dans les valves de la balsamine. Ainsi, en plongeant une de ees valves dans l'eau pure, elle prenait un degré déterminé d'incurvation; si j'ajoutais à l'eau une petite quantité d'acide sulfurique, nitrique ou 
hydro-chlorique, l'incurvation do la valve devenait à l'instant plus profonde; mais l'incurvabilité do cetto valve était altérée, en sorte qu'en la transportant dans du sirop de sucre, elle se redressait, mais sans se rouler en spirale en dehors, comme cela a lieu ordinairement. Si l'action de cet acide affaibli était plus longue, la valve perdait entièrement la faculté de se redresser dans le sirop; son incurvabilité était complètement détruite. Ce phénomène était le résultat de la coagulation du liquide intérieur des cellules, coagulation opérée par l'action de l'acide. Alors les cellules ne contenaient plus un liquide dense, mais simplement un coagulum; elles étaient par conséquent incapables d'exercer l'endosmose, dès lors l'incurvabilité était abolie. L'immersion suffisamment prolongée d'une valve de péricarpe de balsamine, dans l'alcool, produit de même, et par la même raison, l'abolition de son incurvabilité. L'immersion, suffisamment prolongée dans une solution de potasse caustique, anéantit également l'incurvabilité de ces valves, et cela autant par l'altération chimique de leur tissu, que par celle de leurs liquides intérieurs.

Je mis quelques valves de balsamine dans un verre d'eau, à laquelle j'avais ajouté trois gouttes d'hydro-sulfure d'ammoniaque. Les valves se courbèrent d'abord profondément en dedans; deux jours après, leur incurvation était beaucoup diminuée. Je les transportai dans l'eau pure; elles y demeurèrent immobiles. Jo les transportai dans du sirop de sucre; elles se redressèrent jusqu'à la rectitude seulement, et ne se courbèrent point en dehors, 
comme cela a lieu ordinairement : remises dans l'eau, elles affectèrent uno courbure très légère en dedans. Ces valves étaient véritablement dans un état d'engourdissement ou de stupéfaction, et cependant elles avaient conservé leur apparence de vie; elles n'avaient point perdu leur couleur verte, comme cela avait lieu lors de l'abolition de l'incurvabilité de ces valves par des acides, par des alcalis ou par l'alcool.

\section{Mécanisme du mouvement dans les péricarpes du momordica elaterium.}

Le fruit du momordica elaterium, à l'époque de la maturité, se détache de son pédoncule. A l'instant de cette séparation, le liquide contenu dans la cavité centrale du fruit est expulsé avec violence, mêlé avec les graines, par l'ouverture qui provient de la séparation du pédoncule. A la seule inspection de ce phénomène, on peut juger qu'il y a là une contraction des parois de l'organe creux sur le liquide contenu dans sa cavité. J'avais d'abord été porté à douter de ce fait; mais l'observation m'a ramené à le reconnaître. Il ne m'a fallu pour cela que mesurer d'une manière exacte les deux diamètres du fruit ellipsoïde, avant et après son évacuation. Ce fruit, après qu'il a expulsé son liquide central et ses graines par uno violente expulsion, se trouve diminué environ d'un neuvième dans son petit diamètre, et environ d'un douzième dans son grand diamètre. J'ai pris ces mesures d'une manière extrêmement exacte, avec un compas de tourneur. Il n'y a donc point de doute; il y a ici 
une sorte de contraction; l'organe creux s'est resserré sur lui-même dans tous les sens pour expulser le liquide contenu dans sa cavité. Il s'agit actuellement de rechercher le mécanisme de cette contraction afin de savoir si ello offre de l'analogie avec la contraction musculaire des animaux.

Avant sa maturité, le fruit du momordica elaterium ne manifeste ancune tendance à expulser le liquide, alors peu abondant, ciui existe dans sa cavité centrale. Cependant, ce fruit vert donne des marques très sensibles d'incurvabilité. Si l'on en coupe une tranche longitudinale, comme on coupe une côte de melon, cette tranche se courbe profondément sous forme d'un croissant : cette incurvation augmente encore en plongeant la tranche dans l'eau. Si l'on coupe le fruit par tranches circulaires transversales, et qu'on divise chacune de ces tranches circulaires en deux demi-cercles, chacun do ces demi-cercles se courbe profondément, jusqu'à former un petit cercle complet : cetto incurvation augmente par l'immersion dans l'eau. Ainsi, il y a dans lo fruit vert du momordica elaterium une tendance générale à l'incurvation : cette tendance, loin de comprimer le liquide central, tend au contraire à lui faire plus de place, puisque par elle le petit diamètre du fruit tend à s'agrandir. Ce n'est clonc point cette tendance à l'incurvation qui comprime ce liquide, et qui l'expulse à l'époque de la maturité. Effectivement, à cette époque et après l'expulsion du liquide central, les tranches longitudinales du fruit ne tendent plus à se courber en dedans sous forme de croissant. Elles conservent 
leur rectitude, même lorsqu'on les plonge dans l'eau. Ainsi, il y a eu un changement extrêmement notable dans le mode de l'incurvabilité du fruit, comparé dans ses deux états de fruit vert et de fruit mûr. Il s'agit do déterminer, par l'expérience et par l'observation, quel est ce changement survenu.

Le tissu du fruit, examiné au microscope, se trouve spécialement composé do cellules agglomérées. Ces cellules vont en décroissant de grandeur de la circonférence au centre. C'est cette grandeur décroissante des cellules qui se retrouve ici comme dans les valves du péricarpe de la balsamine, qui détermine do même la tendance à l'incurvation en dedans dans le fruit vert; mais cette grandeur décroissante des cellules existe aussi dans le fruit mûr. Pourquoi donc n'existe-t-il plus de tendance à l'incurvation en dedans chez ce dernier? C'est ce que l'observation va dévoiler.

Les cellules qui composent par leur assemblage le fruit du momordica contiennent un liquide organique dense. L'accession extérieure de l'eau ou de la sève lymphatique provoque l'endosmose implétive dans ces oellules, et par suite l'état turgide et l'incurvation en dedans. C'est pour cela que l'incurvation d'une tranche de ce fruit augmente en la plongeant dans l'eau. Si on la plonge dans du sirop do suere, la densité de ce liquide, plus considérable que la densité du liquide intérieur des cellules, provoquera l'endosmose déplétive dans ces cellules, et il en résultera que la tranche perdra son incurvation en dedans, et prendra une incurvation en dehors. Si l'on répète co jeu d'incurvations alter- 
natives dans l'eau et dans lo sirop, il arrivera à la tranche du fruit ce qui est arrivé dans la même expérience à la valve do péricarpo de la balsamine; elle perdra la faculté de prendre de l'incurvation en dedans, en conservant celle do se courber en dehors. C'est lo résultat do la soustraction du liquide dense que contenaient les cellules, soustraction qui a été opérée par l'effet continué de l'endosmoso déplétive. Or, comme il arrive, lors de la maturité du fruit du momordica, qu'il a perdu sa faculté de se courber en dedans, et que cependant il conserve ses cellules décroissantes de dehors en dedans, il faut nécessairement que ces cellules aient perdu une grande partie du liquide dense intérieur qu'elles contenaient, lorsque le fruit était vert. L'expérience va dévoiler la cause de cette déperdition.

Le centre du fruit du momordica elaterium contient une substance organique très singulière, qui ne ressemble à aucun autre tissu végétal. On. le prendrait pour un mucus vert fort épais. $V u$ au microscope, il paraît composé d'une immense quantité do globules fort petits, agglomérés, tantôt confusément, tantôt de manièro à former des stries irrégulières. Cetto substance est pénétrée par un liquide blanchâtre, par une sorte d'émulsion, qui est d'autant plus dense, qu'on l'observe à une époque plus voisine de la maturité. Ce liquide aqueux s'épanche aussitôt qu'on ouvre le fruit vert. Au microscope, on voit des globules presque imperceptibles qui nagent dans ce liquide; à l'époque de la maturité, ce liquide blanchâtre est beaucoup plus 
abondant, et en même temps beaucoup plus dense; les globules qu'il tient en suspension sont devenus beaucoup plus gros. Les graines détachées du fruit nagent dans oe liquide central, qui, par sa densité considérable, provoque l'endosmose déplétive des cellules qui composent le tissu du fruit; dès lors le liquide organique qui remplit ces cellules tend, par l'effet de l'endosmose déplétive, à s'écouler vers lo liquido central, dont la densité est supérieure à la sienne. Cette endosmose déplétive fait cesser la tendance à l'incurvation en dedans, qui existait dans toutes les parties du fruit, qui se trouve alors dans le même cas que s'il était en contact avec du sirop de sucre : ses côtés tendent alor's à la rectitude. La masse du liquide central est augmentée par l'addition du liquido qu'il soutire des cellules. Les côtés du fruit sont courbés mécaniquement en dedans par cette accumulation de liquide dans sa cavité; et comme ces côtés tendent avec force à la rectitude, ils pressent avec violence le liquide central, et ils le chassent rapidement dès qu'une issue lui est ofierte. Cette expulsion n'est pas l'effet do la seule tondance à la rectitude des côtés du fruit; elle est aussi l'effet de la diminution de la capacité de sa cavité centrale, par sa contraction génćrale. Ces deux effets dépendent de la même cause, c'est-à-dire de l'endosmose déplétive des ccllules, produite par l'accession extérieure du liquide central, plus dense que ne l'est le liquide qui remplit ces mêmes cellules. La vérité de cette assertion est prouvée par l'expérience suivante. J'ai pris un nombre suffisant de fruits parvenus 
à leur maturité, et j'ai recueilli dans un vaso le liquide central qu'ils expulsaient, mêlé aux graines; alors j'ai pris un fruit vert, et je l'ai coupé par tranches longitudinales; chacune de ces tranches s'est courbée en croissant, en dedans, comme à l'ordinaire, et cette incurvation s'est augmentée dans l'eau : c'était l'effet naturel de l'endosmose implétive. Alors j'ai transporté ces tranches dans le liquide que j'avais recueilli; elles n'ont pas tardé à diminuer do courbure; ensuito elles se sont redressées complètement; enfin, elles se sont un peu courbées en dehors. Il est prouvé par cette expérience, que le liquide central du fruit mûr agit comme cause d'endosmose déplétive sur les cellules qui composent le tissu du fruit, ce qui prouve que ce liquide est plus dense que no l'est le liquide qui remplit ces cellules. C'est donc l'accession ou le contact de ce liquide central, devenu très dense, qui fait cesser la tendance générale à l'incurvation en dedans, qui existait dans le fruit vert, par l'effet de l'endosmose implétive des cellules, et qui lui substitue une tendance générale au redressement et à l'incurvation en dehors, par l'effet de l'endosmose déplétive de ces mêmes cellules.

Ainsi, il y a deux phases dans l'incurvabilité du fruit du momordica elaterium, savoir : une tendance à l'incurvation en dedans par effet d'endosmose implétive dans le fruit vert, et une tendance à l'incurvation en dehors par effet d'endosmose déplétive dans le fruit mûr. Ce changement ne reconnaît d'autre cause que l'augmentation survenue dans la densité du liquide qui occupe la cavité centrale du fruit. 
Il résulte de ces observations que les valves du péricarpe de la balsamine et le fruit du momordica elaterium possèdent une incurvabilité à,laquelle se joint une sorte de contractilité. L'incurvabilité dépend de la grandeur décroissante des cellules qui composent le tissu incurvable; ce tissu offre, d'un côté, de la capacité en plus, et de l'autre côté do la capacité en moins. Ces cellules contiennent un liquide organique d'une densité toujours supérieure ¿̀ celle de l'eau; lorsqu'elles subissent l'aćcession extérieure de l'eau ou de la sève lymphatique, qui diffère peu de l'eau pure, ces cellules éprouvent l'endosmose implétive, et le tissu se courbe, de manière que les plus grandes cellules occupent le côté convexe. Lorsque ces cellules subissent l'accession d'un liquide plus dense que celui qu'elles contiennent, elles éprouvent l'endosmose déplétive, et il en résulte deux effets; le premier est l'incurvation du tissu, en sens inverse de celui qui avait lieu par endosmose implétive; alors ce sont les plus petites cellules qui sont au côté convexe; le second effet est la contraction ou plutôt le raccourcissement du tissu : c'est le résultat nécessaire de l'évacuation partielle de toutes ses cellules composantes. Par cette déplétion générale, le tissu devient moins volumineux, ou, en d'autres termes, il se contracte, mais cetto contraction n'a rien de commun avec la contraction musculairo des animaux.

\section{§ III. - Mouveurents De LA torsion.}

Les mouvements dont le mécanisme vient d'être étudié s'opèrent par incurvation; ce mode de mou- 
vement est lo plus général chez les végétaux; un second mode de mouvement, qui s'observe moins fréquemment chez eux, est le mouvement de torsion.

Toutes les tiges grimpantes volubiles sont tordues sur elles-mêmes, et j'ai observé que, lo plus souvent, le sens de la spirale que forme la tige par sa torsion sur elle-mêne, est opposć au sens de la spirale que forme la tige volubile autour du support qu'elle' enveloppe. Si ce fait était général, on serait en droit de considérer la disposition volubile de la tige, autour de son appui, comme le résultat nécessaire de sa torsion sur elle-même, dans un sens opposé à celui de sa spire volubile. En effet, l'enchaînement de ces deux phénomènes est facile à saisir. On sait que pour faire une corde composée de deux cordelles, par exemple, on commence par tordre sur elles-mêmes et dans le même sens les deux cordelles, ensuite, en les rapprochant latéralement, on les tord l'une sur l'autre en sens inverse de celui dans lequel est opérée la torsion de chacune des cordelles; alors chacune de ces dernières offre une disposition en spirale qu'elle tend à prendre spontanément par le seul fait de sa jonction à sa congénère, et sans y être forcée par la torsion secondaire qu'opère le cordier et qui ne fait que seconder la disposition naturelle qu'ont ces deux cordelles à être volubiles l'une à l'égard de l'autre. Or, on observe une succession exactement semblable de phénomènes, en associant latéralement deux tiges d'uno planto volubile quelconque, deux tiges de houblon, par exemple. La tige du houblon, vue de l'axe central de cetto tige, se tord sur elle-même 
de droite à gauche; en associant deux de ces tiges qui se développent accolées, elles se disposent l'une sur l'autre en spirale dirigée de gauche à droite, en sorte qu'elles représentent très exactement une corde composée do deux cordelles. Si au lieu d'associer deux tiges de houblon, on associe une seule de ces tiges à un bâton vertical, la tige de houblon enveloppera ce bâton inflexible par sa même spirale dirigée de gauche à droite. C'est exactement pour une seule tige le même phénomène que celui qu'elle présentait lorsqu'elle était associée à une autre tige, sa disposition est la même; le bâton occupe alors l'axe très grossi de la spire; il est entendu que, pour juger de la direction de cette spire, il faut la supposer vue de cet axe; or les mêmes phénomènes s'observent cheż toutes les autres plantes volubiles. Ainsi le chèvrefeuille (linocera caprifolium) tord sa tige sur elle-même de droite à gauche, cette tige est volubile en sens contraire, c'est-à-dire de gauche à droite de même que le houblon; il en est de même $\mathrm{du}$ tamus communis. Le convolvulus purpureus (ipomea purpurea Lam.) tord sa tige sur elle-même do gauche à droite; cette tige est volubile de droite à gauche; la même chose a lieu chez le convolvulus sepium et chez le convolvulus arvensis; mais cependant il arrive souvent que chez ces deux derniers convolvulus, la torsion de la tige sur elle-même offre une spirale dirigée dans le même sens que celui de la spirale volubile, c'est-à-dire également de droite à gauche, en sorte que cela infirmerait la règle générale que je viens d'établir touchant l'opposition du sens de la spirale opérée par la torsion de la tige 
sur elle-même et du sens de la spirale opérée par la disposition volubile de cette tige; toutefois, ce fait n'infirme point complètement la loi dont il est ici question, puisqu'il arrive souvent que chez ces coniolvulus. le sens de la torsion de la tige sur ellemêmo est opposé au sens de la spirale volubile de cette tige, en sorte que le cas contraire, qui se présento souvent aussi à l'observation, peut être considéré comme une aberration dont la cause n'est pas connue. Si done l'on admet que la torsion de la tige sur elle-même, dans un sens déterminé, est la cause de la disposition volubile de cette tige en sens opposé, il ne s'agira que de déterminer la cause du premier phénomène pour avoir, par un enchaînement nécessaire, la cause du second. Pour nous faire une idée de la cause à laquelle est due la torsion d'une tige sur clle-même, supposons que le développement en longueur de son système central, soit inférieur au déreloppement en longueur de son système cortical; ce dernier, par le fait de son excès d'allongement, devra ou bien se plisser par plis transversaux ou bien disposer ses fibres obliquement et en spirale en se tordant sur lui-même, et en entraînant avec lui le système central dans sa torsion. J'ai observé le plissement sur lui-même, du système cortical, par l'effet de l'excès de son développement en longueur, chez les racines du lis blanc (lilium candidum), ocla n'a lieu que lorsque ces racines annuelles sont déjà vieillies, et vers le mois de septembre. Co fait, qui prouve que le système cortical peut acquérir plus de longueur que le système central, permet de penser que c'est à une 
cause semblable, mais qui est suivie d'un effet différent, qu'est due la torsion, sur elle-même, de la tige do certaines plantes volubiles. Il me reste à prouver, par l'expérience, qu'un semblablo effet peut être dî̀ à uno semblable cause. Si l'allongement plus considérable dans la partie superficielle que dans la partie centrale est de nature à produire la torsion d'un caudex végétal, lo même effet devra être produit par le raccourcissement plus considérable dans la partie centrale que dans la partio superficielle. Or l'observation m'a fait voir l'existenee de ce dernier fait, lequel implique nécessairement la réalité de l'existenco du premier. Les feuilles calicinales ou les sépales du salsifis (tragopogon porrifolium) sont fort allongées; leur nervure médiane est assez grosse et leur limbe foliacé est très étroit. Ces feuilles étant séparées du calice et abandonnées à la dessiccation, se tordent sur ellesmêmes et représentent alors une sorte de colonne torse. Cela provient de ce que le centre de leur nervure médiane contient do grosses cellules remplies de liquide aqueux, et de ce que la partie la plus superficielle de cette même nervure est occupée par do petites cellules remplies de liquide aqueux et de matière verte. La dessiccation fait perdre plus de longueur aux rangées de grosses cellules centrales qu'aux rangées de petites cellules superficielles, et comme toutes ces rangées sont intimement adhérentes, il en résulte que les plus longues qui sont en dehor's doivent se courber obliquement en spirale, tandis que les plus courtes qui sont en dedans doivent conserver leur disposition en ligne droite, 
nais étre tordues sur elles-mêmes par l'effort que ont, dans co sens, les rangées extérieures qui les ntraînent de force dans ce mourement de torsion. Il me paraît que c'est par un mécanisme analogue yu certaines tiges volubiles se tordent sur ellesnêmes; chez elles la torsion ne provient point, omme chez les sépales du salsifis, d'un excès de accourcissement dans leur partie centrale, mais ien d'un excès d'allongement dans leur partie superficielle, ce qui produit exactement le même ffet, c'est-à-dire, la torsion sur lui-même, du caudex végétal. On voit en effet que dans les tiges volubiles, ordues sur elles-mêmes, la partio centrale n'a point es organes cellulaires déprimés et raccourcis. C'est lone bien réellement la partie corticale qui a pris in excès d'allongement dont l'existence est prouvée par le fait même de la disposition oblique et piralée de ses faisceaux fibreux.

Il arrive quelquefois que le tronc des arbres est ordu sur lui-même, en sorte que les fibres ligneuses sont disposées en spirale; cela est fréquent chez le orunier (prunus domestica); j'ai observé que la orsion de cet arbre avait lieu tantôt de droite à jauche, tantôt de gauche à droite, il ne me paraît jas donteux que cette torsion ne se soit opérée par in mécanisme analogue à celui que je viens d'exooser. Ce sont peut-être ici les couches nouvelles l'aubier qui prennent un accroissement, en lon;ueur, plus grand que celui des couches plus nciennes.

La disposition des tiges en spirale s'opère très videmment quelquefois par un mécanisme différent 


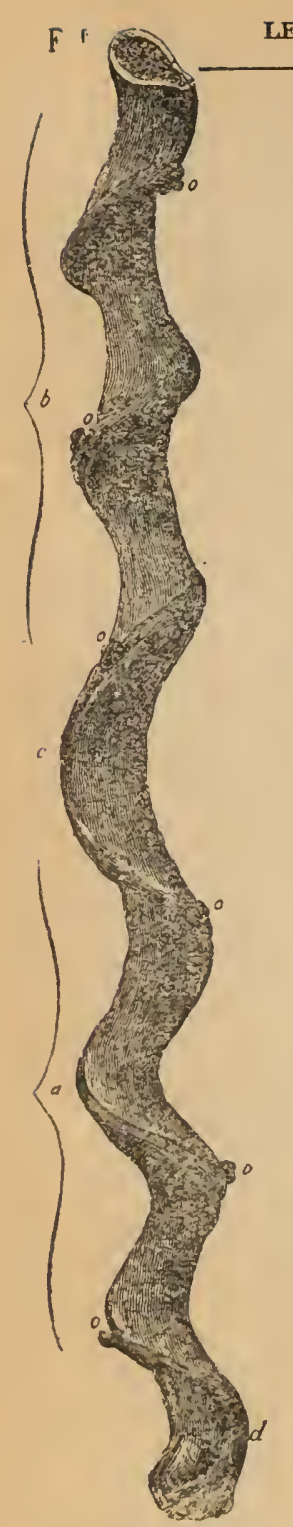

Fig. 1. - Portion de tige de mimosa entada. Sa partie inférieure $a$ est contournee en spirale de gauche à droite, sa partie supérieure $b$ est contournée en spirale de droite a gauche. Les hourgeons 00 suivent la direction de ces deux spirales. de celui que je viens d'indiquer. Le mimosa entada Willd. en offre un exemple très remarquable. Cet arbusto grimpan qui habite les régions intertropicales offre dans sa tige volubile des spires qu sont alternativement dirigées de droit à gauche et de gauche à droite, comm on le voit dans la figure 1. La spire do li partie $a$ de cette tige volubile est dirigé de gauche à droite; cette direction de 1 spire change en $c$ et prend la directios de droite à gauche dans la partie $b$. L même changement de direction de la spir s'observe en $d$, en sorte qu'il est bien évi dent que des spirales inverses occupen alternativement toute la longueur $d$ cette tige volubile. J'ai remarqué qu dans la spire qui s'étend sans changemen do direction de $d$ en $c$, la tige possèd cinq bourgeons, lesquels indiquent ic l'insertion des cinq feuilles dont se com pose la spirale la plus ordinaire, suivar laquelle les feuilles sont disposées sur le tiges végétales. Ici, c'est-à-dire en c figure 1, la spirale des feuilles est dirige de gauche à droite, co qui est aussi sens de la direction de la spira. qu'affecte cette partie de la tige volubil Plus haut, c'est-à-dire en $b$, la spira des feuilles change et se dirige de droi à gauche, ce qui est aussi le sens c la direction do la spirale qu'affec 
cette partie $b$ de la tige volubile ${ }^{(1)}$. Ainsi le changement de direction do la spirale des feuilles entraine ici le changement de direction de la spirale qu'affecte la tige volubile. Cette dernière est forto ment excentrique, comme on le voit par sa coupo transversale représentée par la figure 2. La moelle $a$ entourée d'une mince couche ligneuse concen-

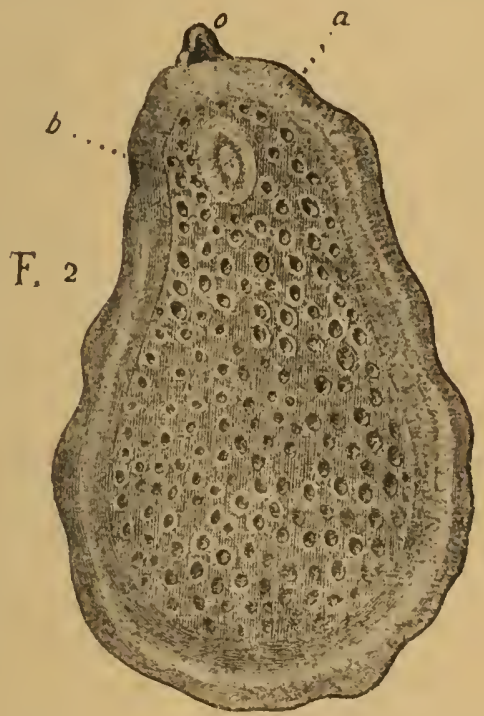

Fr6. 2. - Coupe horizontale de cette même tige qui est très excentrique. Sa moelle est en $a$, elle est recouverte par une couche mince de tissu fibreux $b$. $o$, est un des bourgeons de cette tigo.

trique $b$, est placée tout à fait latéralement dans cette tige excentrique dont la partie ligneuse $c$ s'est presque exclusivement développée sur un seul de ses côtés. Le bourgeon o indique la place qu'occupait uno des feuilles. Ainsi, il paraît certain que c'est ici la disposition des feuilles en spirales alternative-

(1) [Voir le Mémoire de Dutrochet, Variations accidentelles de lx disposition des feuilles in Mémoires, t. I, p. 247. Paris, 1837.] 
ment inverses, qui a occasionné le contournement de la tige en spirales qui sont de même alternativement inverses. Il est infiniment probable que dans l'origine, cette tige n'était point excentrique comme elle l'est dans l'exemple qui est figuré ici; elle devait consister alor's seulement dans la partie cylindrique dont la coupe transversale est représentée en $a b$ (ingure 2), partie qui forme seulement lo cordon spiralé et extérieur, qui porte les bourgeons o oo dans la figure 1. Ce cordon spiralé était la tige primitive. Il n'y a point eu d'accroissement en diamètre au côté convexe de cette tige spiralée primitive, côté qui porte les bourgeons et qui par conséquent portait les feuilles, mais, par contre, il s'est opéré un accroissement excessif en diamètre sur cette tige primitive au côté concave de la spir'e qu'elle affecte; c'est cet accroissement en diamètre du côté concave do la tige spiralée qui a produit la partie ligneuse dont la coupe transversale est représentée en $c$ (figure 2), l'écorce $i$ de cette tige a partout à peu près la même épaisseur. Il ne paraît pas facile de déterminer pourquoi c'est exclusivement le côté concave de la tige spiralée qui s'accroît en diamètre. Je mo bornerai i faire observer que ce fait est en contradiction avec tontes les théories qui ont fait dériver les inflcxions spontanées qu'affectent, dans certains cas, les tiges végétales de l'excès de la nutrition de l'un do leurs côtés sur la nutrition du côté opposé, admettant qu'en pareil cas, c'est le côté le plus fortement nourri qui s'allongerait le plus, et qui par conséquent occupcrait. la convexité de la courbure. Ici, c'est l'inverse qui a lieu; c'est le 
:ôté lo plus fortement nourri et lo plus développé yui occupe la concavité de la ccurbure. Pourquoi, hez le mimosa entada, la disposition spiraléo des cuilles détermine-t-clle le contournement en spirale le la tige, tandis que cela n'a point lieu chez tant l'autres végétaux dont les feuilles sont disposées en piralo? Cela tient très certainement à une partimarité d'organisation chez lo mimosa entada, articularité qui ne pourrait être étudiée qu'en bservant ce végétal à l'état de vie. Les vrilles de a brione (brionia alba L.) offrent aussi des spirales uccessives dont le sens est alternativement de droite $i$ gauche et de gaucho ì droite. C'est, je crois, lo seul égétal de nos climats qui offre ce phénomène dont a cause organique est inconnue.

D'après l'observation qui proure que chez le nimosa entada la spirale de la tige volubile est lirigée dans le sens même de la spirale qu'affectent es feuilles, il serait permis de soupçonner qu'il en serait de même chez les autres végétaux dont les iges sont volubiles, mais l'observation ne confirme soint cette prévision. Ainsi, par exemple, chez le amus communis la spirale des feuilles est dirigée le droite à gauche, et la spirale de la tige volubile sst dirigée de gauche à droite. La direction de la pirale des feuilles n'est pas toujours facile à voir ur les tiges volubiles, parce que ces tiges sont touours tordues sur elles-niêmes; mais on peut les camener par la pensée à l'état d'absence de torsion, on observant les rapports des insertions des feuilles avec les lignes spiralées que décrivent sur la tige tordue les fibres qui, sans cette torsion, auraient été ongitudinales. 
Le mécanisme qui préside à l'exécution des mouvements que je viens d'étudier, mouvements dans lesquels la vitalité de la plante ne paraît point intervenir nécessairement, est également celui qui préside à l'exécution des mouvements vitaux des plantes; ces derniers mouvements, en effet, sont tous dus à l'implétion ou à la déplétion d'un tissu organique composé d'organes cellulaires ou plus généralement de petits organes creux, ainsi que je le ferai voir, en sorte que tous ces mouvements vitaux s'opèrent par incurvation ou par torsion. Cependant, je dois dire ici d'avance que ce n'est pas toujours et exclusivement par implétion ou par déplétion d'eau que ces mouvements vitaux s'opèrent; c'est aussi par implétion et par déplétion d'oxygène, ainsi quo jo lo démontrerai.

\section{§ IV. - Mouvements VÉGÉTAUX DUS A L'HYGROMÉTRIE.}

Tous les mouvements purement mécaniques que je viens de passer en revue sont exécutés par des parties vivantes. Or, on observe souvent des mouvements semblables dans des parties végétales mortes qui prennent des positions alternativement inverses, suivant qu'elles sont ou desséchées ou pénétrées par l'humidité. La rechercho du mécanisme de ces mouvements ne m'a pas paru dépourvue d'intérêt. Je me bornerai ici à deux exemples.

La fleur du xeranthemum lucidum a son calice composé de sépales colorés en jauno; ils sont coriaces et persistants comme le sont en général 
ceux des fleurs qui portent le nom vulgaire d'immortelles. Cette fleur se ferme par l'inflexion des sépales vers le centro de la fleur lorsque l'air environnant lui livre de l'humidité, et elle s'ouvre par l'inflexion de ces mêmes sépales vers le dehors lorsqu'elle perd cette humidité acquise. $\mathrm{Ce}$ double mouvement peut s'observer pendant plusieurs années dans ces fleurs mortes que l'on conserve à l'air libre dans un appartement. Ce phénomène provient de oe que les sépales colorés qui exécutent ce double mouvement par les inflexions alternativement inverses de leur base, possèdent, dans cette partie, des cellules décroissantes de grandeur de dehors en dedans : grandes en dehors ces cellules sont plus petites en dedans, et il en résulte que lorsqu'elles sont gonflées par l'eau, elles courbent le tissu qu'elles composent vers le centre de la fleur ou vers le dedans, et que lorsqu'elles sont affaissées par l'évaporation de l'eau qui les gonflait, elles courbent le tissu qu'elles composent vers le dehors: Le mécanisme de ce double mouvement est exactement le même que celui qui préside généralement aux mouvements en sens alternativement opposés que l'on observe chez les végétaux vivants, mais leur cause déterminante n'est point en tout la même. Chez les végétaux vivants la turgescence ou la déplétion des cellules proviennent des variations de l'endosmose; chez les parties végétales mortes la turgescence ou la déplétion des cellules sont des effets d'hygrométrie. Le second exemple que j'ai à citer est relatif aux mouvements qui sont exécutés par les deux valves des gousses des plantes légumineuses, lorsqu'elles 
sont frappées de mort après leur maturité. Alors ces valves se séparent l'une de l'autre, et il arrive assez souvent que chacune d'elles tend à se rouler en spirale. Ce phénomène est surtout remarquable chez le pois vivace (lathyrus latifolius) et chez le haricot (phaseolus vulgaris). Lorsque les valves de la gousse sont séparées l'une de l'autre et qu'elles se dessèchent, elles se contournent en spirale, de manière à former un tube assez semblable pour la forme à une trachée (figure 3). Lorsque oes gousses redeviennent humides, elles quittent leur disposition en tube spiralé et elles reprennent leur forme ordi-

F. 3

Fig. 3. - Valve de gousse de légumineuse desséchée et contournée en spirale.

naire, qui est celle d'un demi-canal ou d'une gouttière. La cause de co double phénomène m'a paru curieuse à rechercher.

Les valves de la gousse offrent intérieurement une membrane lisse et résistante, en dehors elles possèdent un tissu parenchymateux que recouvre l'enveloppe tégumentaire. La membrane interne est composée de fibres courbes, parallèles les unes aux autres, dont la disposition est très oblique par rapport à l'axe longitudinal de la valve, comme on lo voit dans la figure 4 en $a b, a b$. J'ai indiqué ici par des lignes parallèles la direction de ces fibres que l'on ne voit point à l'extérieur; il faut déchirer 
le tissu de la valve pour apercevoir leur existence; uno lanière étroite de valvo humide, enlevée selon la direction de ces fibres, étant desséchée, elle se courbo en dehors, en sorte que sa convexité est occupée par la membrane interne do la valve; si on la mouille de nouveau, elle so courbe en dedans, comme elle l'était primitivement, en sorte que sa concavité est occupée par la membrane interne de la valve. Ces deux phénomènes sont tout à fait indépendants du parenchyme qui occupe l'extérieur de la valve; car ils ont lieu de même lorsque ce parenchyme est crlevé : ainsi ils appartiennent entièrement à la membrane fibreuse de la valve. J'ai démontré plus haut qu'un tissu végétal, qui possède d'un cóté de grandes cellules, et de l'autre côté des cellules plus petites, se courbe alternativement dans les deux sens opposés, suivant l'implétion ou la déplétion de ses cellules; or, l'observation microscopique fait voir que la membrane fibreuse de la valve est composée de tubes fibreux inégaux en grosseur; les plus extérieurs, ceux qui touchent au parenchyme cortical de la valve, sont plus gros que les tubes fibreux qui sont situés au-dessous et qui touchent à l'épiderme intérieur et extrêmement fin de cette même valve; il résulte de là qu'en imbibant d'eau le tissu membraneux que ces tubes fibreux forment par leur assemblage, ce tissu membraneux se courbera, comme l'expérience le démontre, de manière à ce que les plus gros tubes fibreux occupent la convexité de la courbure, et les plus petits tubes fibreux la concavité de cette même courbure. Lorsque ce tissu membraneux viendra à se dessécher, 
une courbure inverse de ce tissu aura lieu. Ces faits, donnés par la théorie et confirmés par l'observation, étant établis, on voit facilement comment ils produisent tour à tour la disposition de la valve en gouttière et sa disposition en tube spiralé.

La valve est pliée en forme de gouttière dans son état normal; cette courbure a lieu dans le sens $a c$, $b d$ (fig. 4) do la largeur de la valve; or, lorsque cette dernière se dessèche, ses fibres courbes parallèles $a b, a b$, tendant à se redresser, et ne pouvant

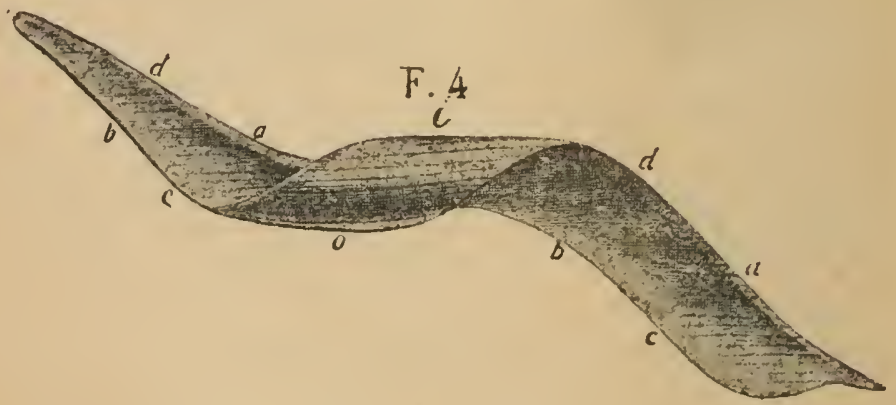

F19. 4. - La nénie valve à moitié déroulée, pour faire roir le mécanisme de son contournement.

opérer cette action, à cause de la grande obliquité de leur courbure particulière avec la courbure en gouttière de la valve; ces fibres parallèles, dis-je, sont ámenées par le fait même de leur tendance au redressement, à prendre dans leur ensemble la seule disposition dans laquelle ce redressement puisse avoir lieu; cette disposition est celle dans laquelle la valvo est roulée en spirale tubuleuse; alors ces fibres deviennent droites, commo on le voit dans la partie io de la valve, qui offre un commencement de la formation du tube spiralé, formation qui est 
simultanée dans toute l'étendue de la valve qui se dessèche et que j'ai représentée seulement dans le milieu do cette dernière, afin de mieux faire saisir le mécanisme au moyen duquel s'opère la disposition de la valve en tube spiralé (fig. 3). Dans ce tube, les fibres parallèles de la membrane fibreuse de la valve sont devenues droites et disposées dans lo sens de la longueur du tube; elles tendent fortement à outrepasser leur redressement et à se courber en dehors, mais leur association, sous forme membraneuse, y met obstacle. Si l'on mouille ce tube spiralé, ses fibres parallèles et droites tendront à reprendre leur courbure primitive en dedans; dès lors la disposition, en tube spiralé, disparaîtra, et la valve reprendra sa forme primitive ou sa forme en gouttière. Ces deux phénomènes alterneront autant de fois que la valve sera alternativement desséchée et mouillée; c'est un effet d'hygrométrie et en même temps l'effet d'un mécanisme assez curieux d'organisation.

En comparant cette dernière observation à celles qui ont été exposées plus haut, touchant le mécanismo qui préside à la disposition spiralée chez les végétaux, on voit que les causes qui opèrent cette disposition spiralée ne sont point les mêmes dans toutes les circonstances où cette disposition se manifeste. 



\section{DU RÉVEIL}

\section{ETT DU SOMNEIL DES PLANTES(1)}

§. I. - DU RÉveil ET DU SOMMEIL DES FLEURS.

Lorsqu'une fleur présente successivement les deux états d'épanouissement et d'occlusion, on dit que le premier do ces deux états est son réveil et que le second est son sommeil. Des fleurs, en très grand nombre, ne présentent point de sommeil; elles s'épanouissent et conservent cet état jusqu'à la mort de la corolle qui se flétrit et tombe sans s'être préalablement fermée; il y a des fleurs qui n'ont qu'un seul réveil qui est leur épanouissement, et qui n'ont qu'un seul sommeil qui précèdo immédiatement la mort de la corolle : telles sont les fleurs des mirabilis, des convolvulus, etc.; il y a cnfin des fleurs qui présentent pendant plusieurs jours les alternatives des deux positions de réveil et de sommeil. Les fleurs de beaucoup de synanthérées sont dans ce cas.

Les phénomènes du réveil et du sommeil des plantes, et spécialement de leurs fleurs, ont frappé très certainement dans tous les temıs les yeux les

(1) Ce mémoire, inédit jusqu'à ce jour, a été communiqué à l'Académie des Sciences do l'Institut dans ses séances des 14 et 21 novembre 1836 . 
moins observateurs; mais c'est Linné qui, le premier, les a étudiés, sous le point de vue scientifique, sans cependant avoir recherché leur cause. Il s'est contenté de noter les heures diverses auxquelles les fleurs s'épanouissent et se ferment; c'est avec le résultat de ces observations qu'il a composé ce qu'il a nommé l'horloge de flore, et qui est connue de tous ceux qui s'occupent de l'étude des plantes. Le réveil et le sommeil des fleurs n'a été étudié depuis que par M. de Candolle (1). Cet habile observateur, en soumettant pendant la nuit, à une lumière artificielle, des fleurs susceptibles de sommeil, et en les mettant, pendant le jour, dans l'obscurité, parvint quelquefois à intervertir les époques de leur réveil et de leur sommeil. Ainsi il vit des fleurs de mirabilis jalappa s'ouvrir le matin et se fermer le soir, ce qui est l'inverse de ce qui a lieu dans l'état naturel; il vit l'ornithoyalum umbellatum ouvrir constamment ses fleurs, à l'heure quelconque, où il se trouvait soumis à l'influence de la lumière artificielle et les fermer lorsqu'il était replacé dans l'obscurité. Malgré beaucoup d'irrégularités, que M. de Candolle observa chez beaucoup d'autres fleurs soumises aux mêmes expériences, l'ensemble des faits lui démontra que la présence et l'absence alternatives de la lumière sont les causes véritables des mouvements qu'exécutent les fleurs pour prendre les positions qui constituent leurs états successifs de réveil et de sommeil; mais il ne détermina point comment la lumière agit, par sa présence, pour

(1) Influence de la lumière artificielle sur les végétaux (Mémoires de l'Institut, savants étrangers, t. I, p. 335). 
produire l'un de ces phénomènes, ni comment son absence détermine le phénomène opposé. En 1831 j'exposai quelques expériences sur ce point, si obscur et cependant si important, do la physiologie végétale; je fis voir que dans le vide de la pompe pneumatique, les fleurs suscoptibles de sommeil et de réveil conservent constamment celui de oes deux états qu'clles possèdent lorsqu'elles y sont placées. En vain alors une fleur, dans l'état de sommeil, est exposée à la lumière et même aux rayons solaires, elle ne quitte point cet état; en vain alors arrive l'obscurité de la nuit, elle ne détermine point le sommeil d'une fleur qui a été placée dans le vide pendant son état de réveil. J'ai fait ces expériences sur les fleurs du leontodon taraxacum, du sonchus oleraceus et du convolvulus arvensis. Ces fleurs avaient alors leurs pédoncules plongés dans l'eau, afin d'entretenir leur vio et leur fraîcheur, les deux premières, qui appartiennent aux synanthérées et qui vivent pendant plusieurs jours, ont pu seules 'm'offrir la persistance $d u$ sommeil des fieurs dans le vide; la dernière, dont les fleurs ne vivent qu'un seul jour, n'a pu m'offrir que la persistance dans le vide de l'état de réveil, car leur sommeil est également persistant à l'air libre; il précède la mort de la corolle. J'avais conclu do ces observations, isolées et fort incomplètes, que l'air contenu dans les organes aérifères ou pneumatiques des plantes joue un rôle important dans les phénomènes de leur réveil et de leur sommeil. Les observations qui vont suivre confirmeront ce premier aperçu.

Pour arriver à la connaissance du mécanisme 
intime qui préside aux actions organiques qui produisent le réveil et le sommeil des fleurs, j'ai dirigé d'abord mes recherches sur les fleurs qui n'offrent qu'un seul réveil qui est leur épanouissement et qu'un seul sommeil qui précède la mort de leur corolle. Parmi les fleurs qui sont dans ce cas, j'ai choisi pour sujets d'étude la fleur du mirabilis jalappa, du mirabilis longiflora, et celle de l'ipomea purpurea Lam. (convolvulus purpureus L.).

On sait que les corolles des mirabitis (belles de nuit) s'épanouissent le soir et se ferment le lendemain matin, lorsque la lumière devient vive. Mais elles restent épanouies jusque dans l'après-midi, si le soleil est caché par d'épais nuages, en sorte qu'il paraît bien évident que c'est la lumière qui détermine l'ocelusion ou le sommeil de leur corolle. La fleur de l'ipomea purpurea s'épanouit vers le milieu do la nuit, et elle conserve cet état de réveil jusqu'au soix du jour qui suit; alors arrive'son occlusion ou son sommeil, qui a lieu exactement de la même manière que celui de la corolle des mirabilis, c'est-à-dire en roulant les bords de la corolle en dedans ou vers son centre. J'ai fait marcher de concert mes expériences sur le mécanisme organique qui opère le réveil et le sommeil de l'ipomea purpurea, du mirabilis jalappa et du mirabilis longiflora. Il y a, comme on s'en doute bien, une similitude parfaite sous le point de vue de co mécanisme organique entre les deux mirabilis dont le réveil est nocturne; mais l'on sera étonné de voir que ce mécanisme est exáctement le même chez l'ipomea dont lo réveil, nocturne d'abord, se prolonge pendant 
toute la durée du jour. Je commence par étudior la fieur du mirabilis jalappa que je prends pour specimen, tout ce que j'en dirai s'appliquant do même au mirabilis longiflora.

La corolle infundibuliforme et monopétale des mirabilis peut être considérée comme formée par la soudure de cinq pétales qui ont chacun leur nervure nédiane. Ces cinq nervures, qui font saillie à la faco externe de la corolle, soutiennent le tissu membraneux de cette dernière comme les fanons de baleine d'un parapluie en soutiennent l'étoffe, et ce sont elles exclusivement qui, par leurs incurvations spontanées, opèrent l'épanouissement de la corolle ou son réveil, et qui opèrent subséquemment son occlusion ou son sommeil; dans le premier cas, les cinq nervures se courbent en dehors, dans le second elles se courbent en dedans en se plissant en zigzag, et par ce mécanisme elles entraînent avec elles lo tissu membraneux de la corolle jusqu'à l'orifice de son canal tubuleux où ce tissu membraneux demeure chiffonné et en bouchon. Ce plissement en zigzag cles nervures est produit, comme on le verra plus bas, par leur tendance à l'incurvation en spirale en dedans. Le tissu membrancux de la corolle auquel les nervures sont organiquement liées, les empêchant do se rouler en spirale uniforme en dedans, les diverses portions de leur longueur so courbent isolément, en sorte qu'il y a une certaine quantité d'arcs les uns à la suite des autres et tous produits par une tendance à l'incurvation dans le même sens, c'est-à-dire en dedans. Ces arcs, dans les endroits où ils sont contigus, forment des angles, ou pré- 
sentent des flexions de la nervure en sens opposé à oelui de l'incurvation spontanée de chacun de ces arcs; mais ces flexions de la nervure ne sont point des incurvations spontanées; co sont des flexions opérées mécaniquement par l'effet des obstacles qui s'opposent à l'incurvation en dedans do la nervure suivant une spirale régulière et concentrique, et qui ne permettent que l'incurvation irrégulière des diverses portions de son étendue. Ainsi la nervure fléchie en zigzag ou courbée sinueusement possède cet état, parce que la moitié du nombre de ses arcs possède une incurvation spontanée qui résulte de l'organisation de ces arcs, et parce que l'autre moitié du nombre de ses arcs, tournés en sens inverso des premiers, possède une flexion mécanique opérée de force et contradictoirement au mode naturel de tendance à l'incurvation que possècle généralement la nervure. Le plissement en zigzag des nervures n'est donc point le produit direct de l'action organique, laquelle ne tend, dans le cas dont il s'agit, qu'à courber régulièrement les nervures en spirale concentrique en dedans.

Avant l'épanouissement ou lorsque la fleur est encore en bouton qui doit s'épanonir sous peu, les mêmes nervures sont aussi légèrement courbées en dedans, en sorte que la portion de la cornlle qui doit subséquemment être évasée est alo:s renflée en massue. Les figures 1,2 et 3 représentent les trois états successifs de bouton, d'épanouissement et d'occlusion de la corollo du mirabilis jalappa. Pour parvenir à la connaissance du mécanisme organique au moyen duquel s'opèrent les inflexions successives 
des nervures de cette corolle, inflexions auxquelles sont dus les trois états successifs qu'elle présente, j'ai dû d'abord étudier la structure intérieure de zes nervures. Ayant donc isolé une de ces nervures du tissu membraneux de la corolle, je l'ai fondue en deux longitudinalement et dans le sens du diamètre do la corolle, et j'ai sournis au microscope cette

F. 1

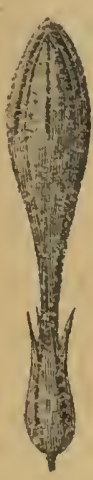

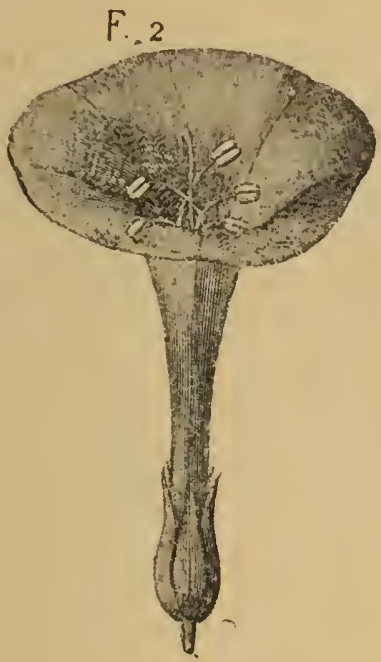

F. 3

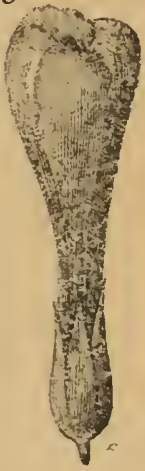

FIG. 1. - Fleur en bouton de mirabilis jalappa.

Fig. 2. - Même fleur épanouie, ou dans l'état de réveil.

F'rs. 3. - Mrême fleur fermée, ou dans l'état de sommeil.

moitié transparente de nervure couverte d'un peu d'eau afin d'augmenter sa transparence. Je dois dire que, pour plus do facilité, je me suis adressé à la variété de cette fleur qui est panachée de rouge et de blane, en sorte que ce sont des nervures incolores que j'ai observées d'abord. Lorsque les nervures sont rouges, leur transparence est moindre, mais je l'ai rendue égale à celle des nervures incolores en 
mettant la nervure rouge dans une solution de potasse caustique qui fait disparaître cette couleur, en sorte que l'observation microscopique ne rencontre plus d'obstacles. La figure 4 représente l'organisation intérieure de cette nervure de corolle : $a$ est son côté externe, $b$ est son côté interne(1). Le côté externe est occupé par un tissu cellulaire $c$

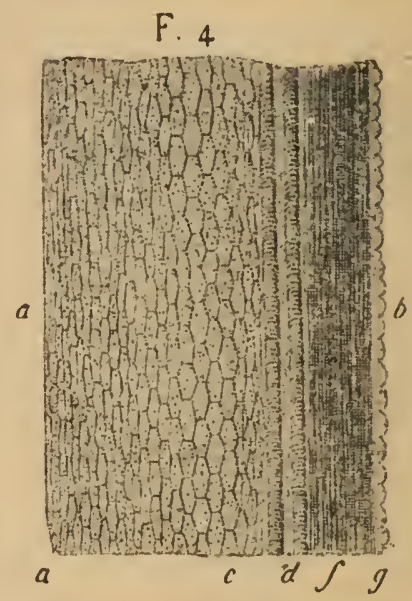

FIG. 4. - Vue au microscope de la coupe longitudinale d'une des nervures de la corolle du mirabilis jalappa; $a$, côté externe; $b$, côté interne; $c$, tissu cellulaire incurvable par turgescence de liquide; $d$, tubes pneumatiques; $f$, tissu fibreux incurvable par turgescence d'oxygène; $g$, cellules pneumatiques superflcielles.

dont les cellules, disposées en séries longitudinales, offrent sur leurs parois une assez grande quantité de globules. A partir des deux tier's intermes $c$ de l'épaisseur de ce tissu, les cellules vont en décroissant de grandeur vers le dehors $a$ et vers le dedans

(1) Cette figure 4, qui représente l'organisation intérieure d'une nervure, deviait être renversée de gauche à droite pour la mettre en harmonie de position avec les nervures isolées que l'ón voit dans les figures $5,6,7,8$ et 9 . 
où elles sont contiguës à des trachées $d$. De l'autre côté do ces dernières, ou à leur côté interne, existe un tissu fibreux $f$ dont la texture extrêmement fine, est très difficile à démêler au microscope. Ce tissu est composé de fibres transparentes entremêlées de globules semblables à ceux qui existent sur les parois des cellules, ces globules sont disposés en séries longitudinales. Ce tissu fibreux correspond en dehors, aux trachées $d$, et en dedans il est recouvert par uno couche de cellules mamelonnées g qui, pour la plupart, sont remplies d'air. Ainsi ce tissu fibreux $f$ est compris entre deux plans d'organes pneumatiques; aussi allons-nous voir que l'oxygène est éminemment nécessaire à ce tissu pour opérer l'incurvation qui lui est propre, incurvation dont on ne peut prévoir le sens; car on n'aperçoit aucun décroissement de grosseur dans les fibres extrêmement petites dont il est en majeure partie composé. Il n'en est pas de même pour le tissu cellulaire $c$. Les deux ordres de décroissement qu'offre la grandeur de ses cellules vers le dehors $a$ et vers le dedans où sont les trachées $d$, indiquent que ce tissu cellulaire est susceptible de se courber en dirigeant sa concavité vers le dedans ou vers le dehors de la corolle. Or, comme l'épaisseur de la couche de cellulès dont la grandeur décroît vers le dehors de $c$ vers $\dot{a}$ est plus grande que ne l'est l'épaisseur de la couche de cellules qui décroissent de grandeur vers le dedans de $c$ vers $d$, il en résulte que, lors de la turgescence de ces cellules, le tissu total qu'elles composent doit se courber en dirigeant sa concavité vers le dehors $a$. Il est à noter que c'est la nervure 
de la corolle épanouie qui est observée ici; cetto même nervure, observée douze heures au moins avant l'épanouissement, offre au contraire dans sa couche de cellules décroissantes de grandeur de $c$ vers $d$ plus d'épaisseur que dans sa couche $c a$, en sorte qu'alors le tissu cellulaire total qui s'étend de a en $d$ doit tendre à se courber en dirigeant sa concavité vers le dedans $b$, lorsque les cellules qui le composent sont dans l'état de turgescence. C'est aussi ce que l'expérience démontre, ainsi que je vais le faire voir.

Dans un jour de l'été, j'ai cueilli à cinq heures du matin une fleur en bouton du mirabilis jalappa,

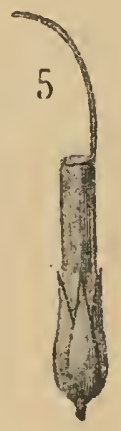

Fig. 5. - Fleur en bouton de mirabilis jalappa, à laquelle on a enlevé toute la partie évasée de la corolle, en ne laissant subsister qu'une seule des nervures, laquelle se courbe en dedans étant plongée dans l'eau.

laquelle devait s'épanouir le soir (figure 1), et j'ai isolé une des nervures de sa corolle jusqu'à l'endroit où elle commence à se rétrécir en tube (figure 5). Cetto nervure courbée en dedans, ayant été plongée avec ce qui reste ici de la fleur dans l'eau, sa courbure en dedans augmenta considérablement. Alors ses 
cellules étaient turgescentes par l'effet de l'endosmose implétive. Je transportai cette fleur ainsi préparée dans du sirop de sucre. La nervure courbée en dedans se redressa d'abord, puis so courba en dehors. Alors ses cellules s'étaient désemplies ou vidées en partie par l'effet de l'endosmose déplétive, le sirop étant plus dense que le liquide intérieur de ces cellules. Il résulte do cette expérieńce que, dans l'état naturel, la nervure de la corolle en bouton est courbée en dedans par l'effet de l'endosmose implétive qui rend ses cellules turgescentes.

Le même jour à deux heures après-midi, j'ai cueilli une nouvelle fleur en bouton devant de même

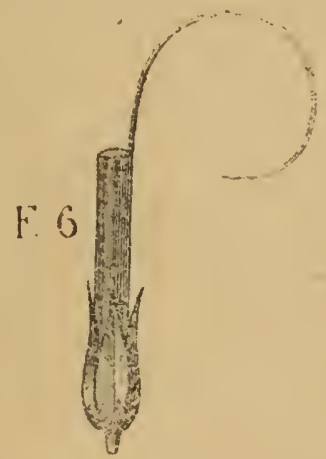

Fig. 6. - Même fleur épanouie traitée comme la précédente. Plongée dans l'eau, sa nervure se courbe en dehors.

s'épauouir le soir, et j'ai isolé une de ses nervures, commo dans l'expérience précédente. Cette nervuro était dans l'air, courbée en dedans, mais l'ayant plongée dans l'eau elle se redressa d'abord et ensuite so eourba profondément en dehors (figure 6), ello agit alors comme elle l'eât fait naturellement lo soir pour épanouir la corolle. L'ayant transportéo 
dans du sirop de sucre, la nervure courbée en dehors se redressa et se courba ensuite en dedans. C'était alors le résultat de la déplétion de ses cellules par l'effet de l'endosmose déplétive; ainsi la courbure en dehors de cette nervure (fig. 6) était le résultat de la turgescence de ses cellules par l'effet de l'endosmose implétive. C'est donc, dans l'état naturel, l'endosmose implétive des cellules provoquée par l'abondance de la sève lymphatique qui produit l'épanouissement de la corolle. Si cet épanouissement n'a lieu que lo soir, cela provient évidemment de ce que la diminution de la lumière et celle de la chaleur occasionnent la diminution de la transpiration végétale, ce qui favorise l'accumulation de la sève lymphatique dans le tissu organique de la corolle. L'eau dans laquelle j'avais plongé à deux heures après-midi la nervure de la corolle en bouton, avait produit prématurément pour cette nervure l'accumulation dans son tissu de la sève lymphatique et par suito avait provoqué le mouvement d'incurvation qui préside à l'épanouissement.

La nervure de la corolle en bouton cueillie le matin (fig. 5), et la nervure de la corolle en bouton cueillie dans l'après-midi du même jour (fig. 6), affectant des courbures inverses, lorsque l'endosmose implétive rend leurs cellules turgescentes, cela prouve que pendant le peu d'heures qui se sont écoulcés entre ces deux époques, il s'est opéré un changement dans l'organisation des nervures do la corolle. Ce changement est une augmentation du développement de la couche cellulaire qui s'étend do $a$ en $c$ (fig. 4), couche qui devient alors plus 
épaisso que la couche cellulaire quii s'étend de $c$ en $d$. Il en résulte que l'incurvation qui, dans ce tissu turgescent, s'opérait auparavant do manière à co que sa concavité fût dirigée vers le dedans $b$ do la corolle, s'opère actuellement dans le sens opposé, c'est-à-dire que la courbure de ce tissu turgescent a lieu de manière à ce que sa concavité soit dirigée vers le dehors a de la corolle; on sera peu étonné de la rapidité de ce changement organique on observant la vitesse avec laquelle s'opère le développement de la corolle dans les heures qui précèdent son épanouissement.

Le tissu cellulaire dont l'épaisseur s'étend de a en $d$ (fig. 4) est ainsi l'agent de l'incurvation, en dedans, des nervures (fig. 5) dans la corolle en bouton, et il est également l'agent de l'incurvation, en dehors, de ces mêmes nervures (fig. 6) dans la corolle épanouie. La turgescence des cellules, par implétion d'eau ou par endosmose implétive, est, dans l'un et dans l'autre cas, la cause do l'incurvation. Il s'agit actuellement de savoir quel est l'agent de l'incurvation nouvelle, en dedans, de ces mêmes nervures lors de l'occlusion de la corolle : c'est ce que l'observation va dévoiler.

Je viens de faire voir que la nervure de corolle, épanouio ou voisine de l'épanouissement, étant isolée et plongée dans l'eau, se courbe en dehors (fig. 6). Or, en laissant dans l'eau cette nervure ainsi courbée, on la voit au bout de six ou huit heures quitter cette courbure et commencer à prendre, par son sommet, uno courbure inverse 
(fig. T); cette nouvelle incurvation augmente assez vite (ifg. 8), en sorte que cette nervure isolée se trouve toute entière roulée en spirale, vers le dedans de la corolle. C'est là l'incurvation qui, dans l'ćtat normal, opère l'occlusion de la fleur ou son sommeil (fig. 3). Certes, c'est un phénomène bien singulier que celui qui est présenté ici par une nervure da corolle isolée et plongée dans l'eau; cette nervure, prise dans la fleur en kouton, voisine de l'épanouissement et encore courbée en dedans, étant
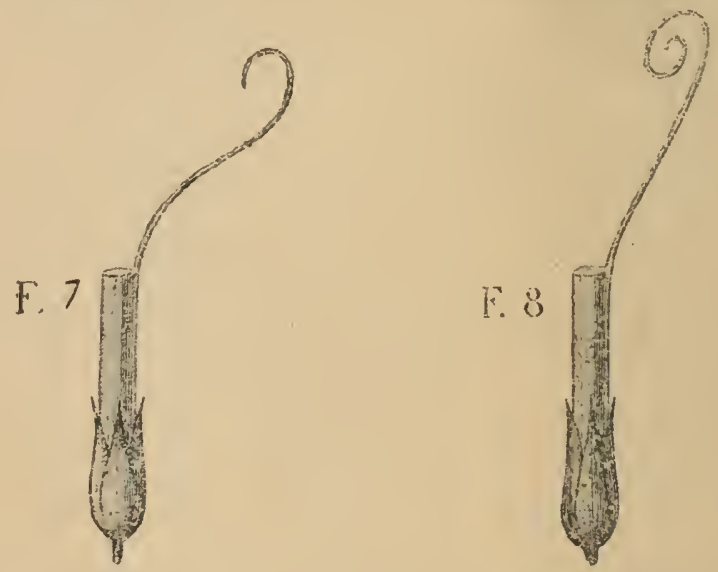

Fig. 7 et 8. - La nelvure qui, plongée dans l'eau, s'était d'abord courbée en dehors, comme on le voit clans la figure 6 , abandonne cette courbure au bout de quelques heures et se courbe en spirale en dedans, comme le représentent les deux figures 7 et 8 .

plongée dans l'eau, elle y prend immédiatement la courbure qui, dans l'état naturel, opère l'épanouissement ou le réveil de la corolle, et ensuite elle y prend de même spontanément la courbure inverse qui est celle qui, dans l'état naturel, opère l'occlusion ou le sommeil de la corolle. Cette succession do phénomènes est tout à fait indépendante do 
l'action de la lumière, car elle a lieu de même dans l'obscurité complete. J'ai fait voir que la courbure qui opère l'épanouissement (fig. 6), chez la nervure plongée dans l'eau, est due à l'endosmose qui introcluit ce liquide dans ses cellules de manière à les lendre turgescentes; mais comment se fait-il que la nervure, qui s'est ainsi courbée dans l'eau, y prenne subséquemment une courbure inverse (fig. 8)? à coup sûr il n'est point survenu de changement d'organisation dans cette nervure isolée, de manière à donner lieu, chez elle, ì une incurvation dans un sens nouveau, ainsi que cela a eu lieu précédemment pour la nervure do fleur cueillie le matin (fig. 5) comparée à la nervuro de fleur cueillie après-midi (fig. 6). J'ai fait voir, en effet, que ces deux nervures, isolées et plongées dans l'eau, se courbent par endosmose implétive dans des sens inverses, en sorte que la présence extéricure de l'eau produit ici deux courbures en sens opposés; j'ai prouvé, par une expérience décisive, que dans ces deux cas, l'incurvation est également due à l'endosmose implétive des cellules. Cette expérience a consisté à plonger ces deux nervures dans du sirop de sucre, lequel, en provoquant l'endosmose déplétive des cellules de ces nervures, occasionna leur courbure dans un sens inverse de celui qu'elles avaient pris dans l'eau. Or, j'ai soumis à la même épreure la nervure (fig. 8) qui avait pris secondairement la courbure spiralće en dedans, quoique toujours plongée dans l'eau, laquelle d'abord avait provoqué sa courbure en dehors; cette nervure courbée en spiralo (fig. 8) conserva dans le sirop cette même courbure, en sorte 
que je fus assuré que cette courbure secondaire n'était point due à l'endosmose; il me parut dès lors certain que cette courbure spiralée n'avait point pour agent le tissu cellulaire, $c$ (fig. 4), mais bien le tissu fibreux $f$ dont la fonction m'était encore inconnue; pour savoir à quoi m'en tenir, à cet égard, je pris une corolle épanouie de mirabilis jalappa, et avec un instrument bien tranchant, je fendis une de ses nervures dans le sens de son épaisseur, de manière à séparer le tissu cellulaire $c$ du tissu fibreux $f$; cette opération n'est pas très difficile à pratiquer, parce que la nervure fait saillie à la face externe de la corolle, en sorte que cette

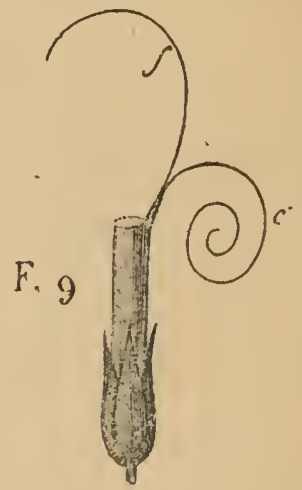

Fit. 9 - Même fleur, dont une nervure a été fendue en deux; la moitié externe $c$ se courbe en clehors, et la moitié interne $f$ se courbe en dedans.

saillie, spécialement formée par le tissu cellulaire c, peut être détachée par la section du tissu fibreux $f$ qui demeure alors dans l'épaisseur de la corolle. Cette opération faite, j'enlevai le tissu membraneux adhérent à droite et à gauche à la moitié de nervure qui contenait le tissu fibreux $f$, et je plongeai dans l'eau cette nervure isolée et fendue en deux (figure9). 
A l'instant, la moitié externe $c$ de cette nervure so courba fortement en dehors et se roula même en spirale. Cette moitié cornposée par le tissu cellulaire $c$ (figure 4) est donc celle qui, par l'endosmose implétive de ses cellules, courbe la nervure en dehors pour opérer l'épanouissement; quant à la moitié interne $f$ (figure 9) de la nervure, elle se courba en dedans, ce qui me fit voir que cette moitié interne, formée par le tissu fibreux $f$ (figure 4), est l'agent de la courbure secondaire de la nervure en dedans pour opérer l'occlusion de la corolle. L'expérience qui vient d'être exposée prouve que les deux courbures en sens inverse du tissu cellulaire $c$ et du tissu fibreux $f$ (fig. 4), tendent en même temps à s'effectuer. Ainsi, lors de l'épanouissement la nervure étant courbée en dehors par l'action d'incurvation du tissu cellulaire $c$, il se trouve que le tissu fibreux $f$ est courbé malgré lui dans ce même sens. Lors de l'occlusion de la corolle, le tissu fibreux $f$ devient à son tour vainqueur de son antagoniste le tissu cellulaire $c$, et il l'entraîne malgré lui dans le sens d'incurvation en dedans qui lui est propre. Je me suis assuré de ce dernier fait en pratiquant sur une fleur dans l'état d'occlusion la division en deux d'une nervure (figure 9), comme je l'avais fait précédemment sur une fleur dans l'état d'épanouissement. Le résultat fut le même; les deux tissus incurvables $c$ et $f$ se courbèrent dans l'eau, le premier en dehors et le second en dedans. Quelle est la cause de cette courbure en dedans du tissu fibreux $f$ ? 11 est évident, par l'expérience rapportée plus haut, que cette cause n'est pas la 
turgescence de ce tissu par endosmose; il y a donc ici un phénomène tout à fait inconnu.

En réfléchissant à ce singulier phénomène, je fus porté à penser que ce n'était point sans raison que la nature avait prodigué les organes respiratoires au tissu fibreux $f$, lequel est situé entre deux plans d'organes creux remplis d'air. Puisque ce n'était pas par implétion de liquide que lo tissu fibreux $f$ prenait son état actif de courbure, ce pouvait être par implétion d'oxygène. Si ce soupçon était fondé, la nervure qui, plongée dans l'eau aérée y prenait d'abord l'incurvation en dehors, qui est celle du réveil, et qui y prenait subséquemment l'incurvation en dedans, qui est celle du sommeil; cette nervure, dis-je, plongée dans l'eau non aérée, devait y conserver invariablement sa première incurvation en dehors qui est celle du réveil, incurvation qui est due à l'endosmose du tissu cellulaire. Cette nervure ne devait, ainsi, jamais présenter l'incurvation en dedans qui est celle du sommeil, et que je pensais devoir être due à l'oxygénation du tissu fibreux $f$ (figure 4). Je dois dire d'abord que, lorsqu'on plonge une partie végétale peu épaisse dans l'eau non aérće, celle-ci dissout promptement l'air contenu dans les organes pneumatiques de cetto partio végétale et prend la place de cet air, en sorte qu'il n'y a plus d'oxygène respiratoire dans cette partie végétale. L'expérience justifia mes prévisions. Une nervure de fieur de mirabitis plongée dans l'eau non aérée y prit et y conserva invariablement son incurvation de réveil. J'avais mis cette eau non aérée qui contenait la nervure en expérience 
dans un flacon bouché avec son bouchon de cristal sans enfermer d'air avec l'eau (1), en sorte que l'eau non aćrée, à l'abri du contact de l'air atmosphérique, n'en pouvait point dissoudre. Cette nervure resta donc constamment courbée en dehors, comme on le voit dans la figure 6 . Elle y devint même beaucoup plus courbée qu'elle ne l'en̂t été dans l'eau aérée. Quant à la courbure secondaire en dedans ou ì l'incurvation de sommeil, telle qu'on la voit dans les figures 7 et 8 , elle ne se manifesta point. Certain par cette expérience que l'incurvation de sommeil ne se manifeste point dans les nervures de corolle du mirabilis plongées dans l'eau non aérée, je voulus voir si le mème phénomène se manifesterait dans une de ces corolles soumise à l'expérience dans son entier. Une corolle de mirabilis épanouie ćtant plongée dans l'eau aérée, elle y prend après quelques heures l'état d'occlusion ou de sommeil; or, j'ai expérimenté qu'une corolle épanouie de cette même plante étant plongéc dans l'eau non aérée mise à l'abri du contact de l'air atmosphérique, elle $\mathrm{y}$ conserve invariablement son état d'épanouissement ou de r'éveil. On pourrait peutêtro penser que l'air contenu dans les organes pneumatiques des nervures de la corolle, agirait en vertu de son élasticité pour produire l'incurvation de sommeil et non en vertu de l'action chimique de

(1) On peut priver l'eau de l'air qu'elle tient en dissolution par le moyen de la pompe pneumatique, ou plus simplement par le moyen de l'ébullition prolongée. Je plonge un flacon dans un vase plein d'eau qui est soumise à l'ébullition; lorsque celle-ci est finie, je mets au flacon submergé son bouchon de cristal, en sorte que l'eau qu'il contient se refroidit sans le contact de l'air, et en même temps que l'eau dans laquelle il est plongé. 
l'oxygène qu'il contient. De là viendrait que l'incurvation de sommeil n'aurait point lieu en plongeant la corolle dans l'eau non aérée qui dissout l'air contenu dans les organes pneumatiques et qui prend sa place; mais cela n'est point ainsi. L'expérience m'a prouvé que l'air ne revient jamais dans les organes pneumatiques envahis par l'eau chez les parties végétales qui continuent à demeurer submergées. Or, cela n'empêche pas une corolle de mirabilis de prendre l'état de sommeil après deux ou trois jours, lorsqu'on laisse l'eau non aérée dans laquelle elle avait été plongée épanouie, s'aérer par son contact avec l'air atmosphérique. C'est donc indubitablement par l'action chimique de l'oxygène dissous dans l'eau, que le tissu fibreux acquiert la force d'incurvation qui produit l'état de sommeil. Ce tissu fibreux $f$ (figure 4) se courbe par implétion d'oxygène comme le tissu cellulaire $c$ se courbe par implétion de liquide. Ainsi, chez la fleur des mirabilis le réveil et le sommeil, c'est-à-dire l'épanouissement et l'occlusion de la corolle résultent de l'action alternativement prédominante de deux tissus organiques situés dans les nervures de la corolle et qui tendent à se courber dans des sens inverses. Savoir : $1^{\circ}$ un tissu cellulaire qui tend à se courber vers le dehors de la fleur par implétion do liquide avec excès ou par endosmose; $2^{\circ}$ un tissu fibreux qui tend à se courber vers le dedans de la fleur par oxygénation. C'est évidemment pour favoriser i'exercice de la fonction d'incurvation de co dernier tissu qu'il se trouve placé entre deux plans d'organes pneumatiques, savoir : un plan de trachées $d$ et un plan 
do cellules aérifères $g$. L'intervention de l'oxygèno est ainsi directement nécessaire pour la production du sommeil; cette intervention de l'oxygène est aussi nécessaire, mais indivectement, pour la production du réveil, parce que cette intervention est nécessaire pour que la corolle attire la sève dans le tissu do ses nervures avec une abondance suffisante pour donner lieu à son implétion par endosmose ${ }^{(1)}$. Ces faits m'expliquent pourquoi une fleur, mise dans lo vide de la pompe pneumatique, $\mathrm{y}$ conserve invariablement l'état de réveil ou de sommeil qu'elle a lor'squ'ello y est placée.

C'est un fait nouveau, et d'une haute importance en physiologie, que celui de l'uxistence d'un tissu qui se courbe par implétion d'oxygène. Ce fait s'appliquera facilement à l'incurvation sinueuse de la fibre musculaire chez les animaux. Mais je reviens à la corolle du mirabilis jalappa: j'ai dit plus haut que la nervure de cette corolle en bouton, cueillie de grand matin, étant plongée dans l'eau, y augmente sa courbure naturelle en dedans (fig. 5); c'est un effet de la turgescence des cellules par endosmose, ainsi que je l'ai démontré plus haut : or, si on laisse cette nervure séjourner pendant quelques heures dans l'eau, on la voit se rouler en spirale dans le même sens, c'est-à-dire en dedans (fig. 7 et 8); ce second effet, tout pareil à celui qui arrive secondairement à la nervure de corolle épanouio plongée dans l'eaul, est dû de même à l'oxygénation, car il n'a point lien dans l'eau non aérée. Ainsi la

(1) Voyez dans le Mémoire VIII, p. 413. [Recherches sur les conduits de la sève et sur les causes de su progression, in Mémoires, t. I.] 
courbure première et immédiate en dedans (fig. 5) a pour agent le tissu cellulaire $c$ (fig. 4), dont l'organisation, à cette époque, est apte à opérer l'incurvation de ce tissu en dedans; la courbure secondaire (fig. 7 et 8 ), qui s'opère de même en dedans a pour agent le tissu fibreux $f$, de même que je l'ai exposé plus haut; la nervure de la fleur en bouton, cueillie le matin et plongée dans l'eau, passe donc immédiatement de la courbure simple en dedans, par endosmose, à la courbure spiralée en dedans par oxygénation, sans passer par l'état intermédiaire de courbure en dehors par endosmose, lequel constitue l'épanouissement ou le réveit. On conçoit, en 'effet, que cette nervure d'une fleur cueillie, a dû perdre 13 progrès de développement cellulaire qui aurait changé lo sens de son incurvation par endosmose, si la fleur eût continué d'être liée organiquement à la plante.

Tous ces phénomènes qui sont communs au mirabilis jalappa et au mirabilis longiflora, le sont également-à l'ipomea purpurea; seulement la fleur de cette dernière plante, lorsqu'elle est en bouton, a ses nervures courbées en spirale en dedans, comme on le voit dans la figure 10, ce en quoi elle diffère, mais légèrement, de la fleur en bouton des mirabilis (fig. 1), chez laquelle les nervures sont simplement courbées en dedans; en outre les cinq nervures do la fleur de l'ipomea purpurea ne sont pas simples, commo le sont les cinq nervures de la fleur des mirabulis; elles sont complexes, c'est-à-dire que chacune de ces cinq nervures, qui sont triangulaires, est composée par la réunion de plusieurs nervures 
armi lesquelles les deux nervures latérales, qui rment les côtés du triangle, so distinguent par ur grosseur; elles font saillio à la face externe de corolle. C'est à ces nervures latérales qu'il faut adresser, tant pour observer leur structure intéieure, qui est tout à fait semblable à celle des ervures des mirabilis (fig. 4), que pour faire des xpériences semblables à celles que j'ai faites sur les ervures de la fleur de oes dernières plantes, et que ai rapportées plus haut. Les phénomènes que j'ai

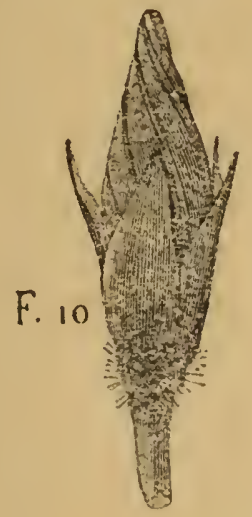

FIG. 10. - Fleur en bouton d'ipomea purpurea.

écrits se passent exactement de la même manière hez les nervures de la fleur de l'ipomea purpurea; on épanouissement ou son réveil est dû à l'incuration en dehors, d'un tissu cellulaire turgescent ar implétion d'eau, implétion due à l'endosmose; on occlusion ou son sommeil est dû à l'incurvation n dedans d'un tissu fibreux turgescent, par implétion 'oxygène : aussi peut-on conserver les fleurs de etto plante, et de même celles de tous les convolulus, dans l'état d'épanouissement ou de réveil, 
en les plongeant dans de l'eau non aérée privée de communication avec l'air atmosphérique; elles demeurent constamment dans cet état, tandis que dans l'eau aérée elles prennent assez promptement l'incurvation spiralée en dedans, qui constitue l'état de sommeil. 'Si on laisse l'eau non aérée, dans laquelle est une de ces corolles épanouies, en contact avec l'air atmosphérique, elle reprendra de l'air en dissolution et deux jours environ après son immersion, la corolle prendra l'état d'occlusion.

Les phénomènes anatomiques et physiologiques rapportés plus haut, étant exactement les mêmes chez la fleur des mirabilis et chez la fleur de l'ipomea purpurea, on se demandera sans doute pourquoi la fleur des mirabilis se ferme le matin, tandis que la fleur de l'ipomea reste épanouie pendant tout le jour. La. réponse à cette question est facile : d'abord jo ferai remarquer que la fleur de l'ipomea purpurea s'épanouit vers le milieu de la nuit, en sorte qu'elle ressemble physiologiquement aux fleurs des mirabilis, sous le point de vue de son épanouissoment lors de la diminution considérable de la lumière. Souvent j'ai vu, dans l'arrière-saison, cette fleur de l'ipomea purpurea s'ouvrir dès dix heures du soir; elle persiste dans l'état d'épanouissement pendant toute la journée qui suit, et elle se ferme dans la soirée. Ainsi, la différence essentiello qui existo physiologiquement, entre cette fleur et celle des mirabilis, consisto en cela que cetto dernière ne supporte pas, sans se fermer, l'action d'une vive lumière, tandis que la seconde, non seulement supporte très bien, sans se fermer, l'action de la plus 
rive lumière solaire, mais ne se ferme ordinairement que lorsque la lumière a commencé à diminuer, 'est-à-dire lo soir; or, cela n'arrive pas toujour's insi, j'ai observé que dans l'automne, lorsque la empérature atmosphérique est descendue à environ 15 degrés C. ou 12 degrés R., les fleurs d'ipomea ourpurea, qui s'épanouissent deux heures environ uprès le coucher du soleil, ne se ferment que dans la natinée du surlendemain; en sorte qu'elles restent panouies pendant environ trente-six heures, tandis que leur état d'épanouissement ne dure que la moitié le ce temps environ lorsqu'il fait chaud. Ce phénonène provient évidemment de ce que l'abaissement le la température augmente la lenteur de l'oxygéıation du tissu fibreux incurvable par oxygénation t agent du sommeil. La lumière, comme la chaleur, xerce de l'influence sur la rapidité de cette oxygéation. Lorsque le soleil est voilé par d'épais nuages, a fleur des mirabilis reste bien plus longtemps uverte; elle ne se ferme alors que dans l'après-midi; ette fleur, mise dans l'obscurité, se ferme encore plus tard. Dans l'ordre ordinaire des choses, cette nême fleur reste ouverte pendant environ douze 1eures, puisque, s'ouvrant vers sept heures du soir lans le mois d'août, elle se ferme vers sept heures lu matin; la fleur de l'ipomea purpurea reste panouie pendant environ dix-huit heures, puisque 'ouvrant, dans le même mois, vers minuit, elle ne ie ferme que vers le coucher du soleil le jour qui suit. Tout atteste done que la fleur des mirabitis est orompte à oxygéner, sous l'influence de la lumière t de la chaleur, son tissu fibreux incurvable qui 
est l'agent du sommeil, et que la fleur de l'ipomea purpurea est lente dans les mêmes circonstances à oxygéner ce même tissu fibreux dont l'incurvation, par oxygénation, produit l'occlusion de la corolle; voilà pourquoi la fleur de l'ipomea purpurea qui, $\mathrm{du}$ reste, s'ouvre cinq ou six heures après l'ouverture de la fleur des mirabilis, ne se ferme qu'environ douze heures après l'occlusion de cette dernière, et reste ainsi épanouie pendant toute la durée du jour.

Il reste actuellement à expliquer pourquoi ces fleurs sont nocturnes sous le point de vue de l'heure de leur épanouissement. Les corolles, devant leur épanouissement à la turgescence d'un tissu cellulaire incurvable par endosmose, doivent offrir ce phénomène à l'époque de la révolution diurne, qui offre les circonstances extérieures les plus favorables pour que cette turgescence ait lieu. Or, c'est généralement pendant l'absence du soleil qu'il y a le plus d'humidité dans l'atmosphère; c'est là une première circonstance extérieure favorable à l'épanouissement des fleurs. Viennent ensuite la lumière et la chaleur. La lumière produit deux effets sur les plantes : $1^{\circ}$ en donnant de l'activité à la respiration végétale, elle augmente l'afflux de la sève ou son ascension par l'attraction (1), et il en résulte un état de turgescenco pour la partie végétale qui est le siège de cet afflux; $2^{\circ}$ la lumière augmente la transpiration végétale, et sous ce point de vue, elle tend à diminuer l'état do turgescence d'eau des parties végétales qu'elle frappe. Ainsi, la lumière est à la fois une

(1) Voyez dans le Mémoire VIII, p. 413. [Rucherches sur les conduits de la sève, in Mémoires, t. I.] 
eauso de turgescence cellulaire par implétion d'eau, et une cause de déplétion d'eau ou de sève lymphatique; suivant que l'une ou l'autre de ces deux influences physiologiques de la lumière sera prédominante, le tissu végétal sera turgescent ou désempli. Supposons actuellement qu'une fleur, par a constitution particulière, soit de nature à éprouver, sous l'influence de la lumière, plus de transpiration qu'eile n'a, sous cette même influence, de forco pour attirer la sève lymphatique : cette fleur ne pourra acquérir la turgescence cellulaire, qui doit opérer son épanouissement, que dans l'absence ou lors de la diminution considérable de la lumière; elle s'épanouira le soir ou pendant la nuit. Supposons que chez une autre fleur il y ait, au contraire, sous l'influenco de la lumière, plus dattraction de la sève lymphatique, qu'il n'y a de transpiration : il y aura alors chez cette fleur turgescence cellulaire, et elle prendra son état d'épanouissement sous l'influence de la lumière. Ces faits donnent l'explication de toutes les différences qui existent entre les fleurs, sous le point de vue de l'époque diurne ou nocturne de leur épanouissement ou de leur réveil. Quant à l'époque de l'arrivée de leur occlusion ou de leur sommeil, elle dépend de la rapidité plus ou moins grande avec laquelle s'opère l'oxygénation de leur tissu fibreux qui est l'agent du sommeil. Il est des fleurs dont lo sommeil arrive très peu de temps après le réveil; il en est d'autres chez lesquelles ces deux phénomènes sont séparés par un espace de temps plus considérable. C'est sur ces différences que sont fondés les phéno- 
mènes que présentent les fleurs, sous le point de vue des heures diverses auxquelles arrivent leur réveil et leur sommeil, phénomènes dont Linné a fait un catalogue sous le nom d'Horloge die flore. Au reste, il faut bien se donner de garde de considérer cette horloge comme exacte, les heures du réveil et $\mathrm{du}$ sommeil des fleurs variant suivant lo degré do lumière, suivant la température, et même souvent suivant l'état de sécheresse ou d'humidité de l'atmosphère.

Les fleurs dont je viens d'étudier le réveil et le sommeil ne présentent qu'une seul fois chacun de oes deux états; il était fort important d'étudier, sous ce point de vue, les fleurs dont la vie est plus longue et qui présentent plusieurs alternatives de réveil ou d'épanouissement et de sommeil ou d'occlusion. Une plante fort commune, le pissenlit (leontadon taraxacum) est celle que j'ai choisie pour faire cette observation. La fleur de cette plante synanthérée vit ordinairement pendant deux jours et demi, en sorte qu'elle présente pendant deux jours le réveil lo matin et le sommeil le soir; le troisième jour elle présente encore le réveil le matin et dans le milieu du jour elle prend son dernier sommeil, qui est suivi de la mort. Dans le réveil, les demifleurons dont cette fleur est composée se courbent en dehors et la fleur est épanouie; dans le sommeil, les demi-fleurons se courbent en dedans et ia fleur est dans l'état d'occlusion. Les demi-fleurons les plus extérieurs, étant les plus grands, sont ceux qui présentent lo plus de facilité pour l'obscrvation. La 
figure 11 représente un de ces denii-fleurons amplifié, et dans l'état de réveil; la figure 12 le r'eprésente dans l'état de sommeil. Chez la plupart des chicoracées, les incurvations inverses et alternatives
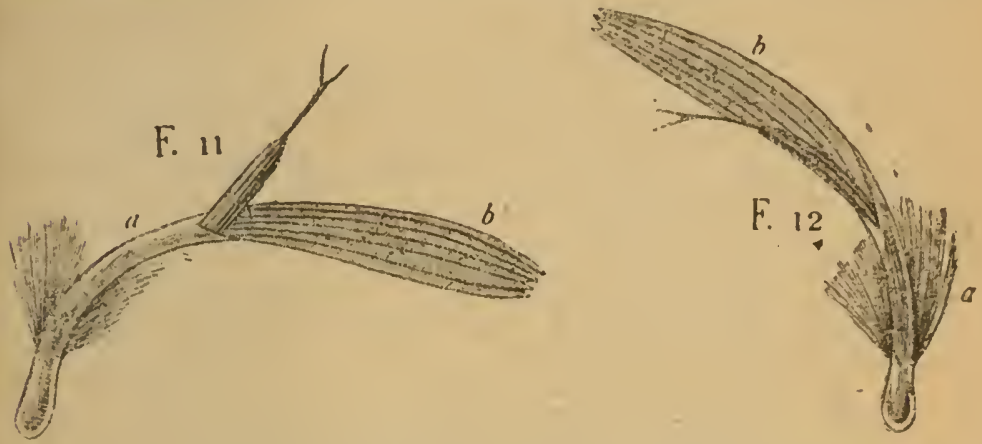

FıG. 11. - Demi-fleuron de la fleur du pissenlit dans l'état de réveil. FrG. 12. - Le même demi-fleuron dans l'état de sommeil.

qui constituent le réveil et le sommeil, ont exclusivement leur siège dans le cornet $a \mathrm{du}$ demi-fleuron, la partie supérieure et colorée $b$ de ce demi-fleuron, ou sa languette, n'y participe point du tout; chez

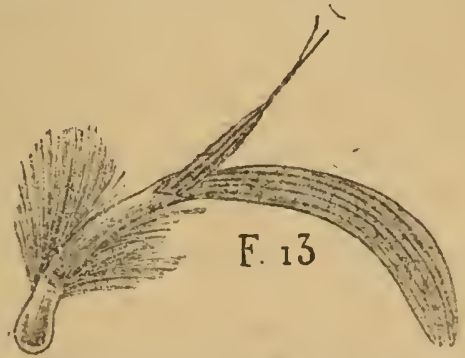

FiG. 13. - Le même demi-fleuron dans l'état de réveil exagéré, tel qu'il a lieu lorsque ce demi-fleuron est plongé dans l'eau non aérée.

le pissenlit le demi-fleuron tout entier se courbe en dehors dans le réveil parfait, et il so courbe de même tout entier en dedans lors du sommeil pro- 
fond. Ainsi, ces demi-fleurons présentent beaucoup de facilité pour l'observation des phénomènes du réveil et du sommeil. L'étude do leur organisation intérieure offre plus de difficulté à raison du peu d'épaisseur des petites nervures qui existent dans le tissu de leur corolle, nervures qui doivent être, et qui sont en effet, les agents des incurvations opposées et alternatives auxquelles sont dus le réveil et le sommeil. Pour observer leur organisation intérieure, il faut déchirer le tissu do la corolle en lanières longitudinales aussi fines qu'il est possible

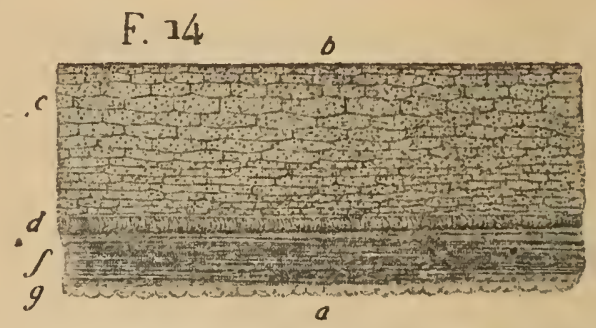

Frg. 14. - Vue au microscope de la coupe longitudinale de l'une des nervures du demi-fleuron de la fleur du pissenlit; $b$, côté interne; $a$, côté externe. $c$, tissu cellulaire incurvable par turgescence de liquide; $d$, tubes pneumatiques; $f$, tissu fibreux incurvable par turgescence d'oxygène; $g$, cellules pneumatíques superficielles.

de les obtenir. De cette manière, on finit par isoler une des petites nervures longitudinales de cette corolle, et comme elle se courbe en cercle en dehors dans l'eau dont on la couvre sur le porte-objet du microscope, il en résulte qu'elle présente le côté à l'œil de l'observateur, en sorte qu'on peut apercevoir son organisation dans le sens de son épaisseur, ce qu'il importait d'obtenir. Voici quelle est cette organisation. La figure 14 représente l'épaisseur d'uno nervure du demi-fleuron; au-dessous de l'épiderme de 
sa face interne ou supérieure $b$, on aperçoit des rangées longitudinales de cellules allongées $c$ disposées en séries rectilignes et couvertes d'une grande quantité de globules de couleur jaunâtre qui s'opposent quelquefois à ce qu'on puisse facilement distinguer les cellules dont ils tapissent les parois. On rend l'observation de ces cellules plus facile au moyen d'un peu de solution de potasse caustique qui rend ce tissu plus transparent. Ce tissu cellulaire $c$ offre ses plus grandes cellules dans la partie de son épaisseur, qui est peu éloignée de la face supérieure $b$ où les cellules superficielles sont plus petites, ce qui établit un léger décroissement de grandeur des cellules de l'intérieur du demi-fleuron vers sa face supérieure $b$. Ce tissu cellulaire, dans le reste de son épaisseur qui est plus considérable, offre le décroissement de grandeur de ses cellules vers un plan de trachées $d$ situées à peu de distance de la face externe ou inférieure $a$ du demi-fleuron. Cette face qui, considérée dans les demi-fleurons de la rangée la plus extérieure, offre dans son milieu une bande longitudinale verdâtre, est occupée superficiellement par des cellules mamelonnées $g$ qui sont, pour la plupart, remplies d'air. Entre ce plan de cellules superficielles $g$ et le plan de trachées $d$, se voit une couche mince de tissu fibreux $f$, lequel est composé de fibres transparentes, entre les faisceaux desquelles se voient des globules excessivement petits. Il est facile de voir, au premier coup-d'œil, que octte organisation intérieure du demi-fleuron est semblable à celle des nervures de la fleur des mirabilis (figure 4), excepté quo la 
disposition des parties composantes y est inverse. Dans la nervure de la fleur des mirabilis et de l'ipomea purpurea, le tissu cellulaire $c$ (figure 4) est voisin de la face externe ou inférieure $a$ de la corolle, tandis que le tissu fibreux $f$, compris entre un plan de trachées et un plan de cellules pneumatiques superficielles, est voisin de la face interne ou supérieure $b$ de cette même corolle. La disposition de ces parties est inverse dans les nervures des demi-fleurons chez lo pissenlit. Le tissu celltlaire $c$ (figure 14) est voisin de la face supérieure $b$, et le tissu fibreux $f$, compris de même entre deux plans d'organes pneumatiques, est voisin de la face inférieure $a$. Cette inversion, au reste, ne change rien aux fonctions de ces parties composantes.

Il est évident que chez le pissenlit, le tissu cellulaire $c$ est le tissu incurvable par endosmose, et que le tissu fibreux $f$ est le tissu incurvable par oxygénation, ainsi que cela a lieu chez les mirabilis. Le premier est, par conséquent, l'organe qui opère l'incurvation en dehors de chaque demi-fleuron (figure 11), incurvation à laquelle est dû l'épanouissement ou le réveil de la fleur. Le second est l'organe qui opère l'incurvation en dedans de chaque demi-fleuron (figure 12), incurvation à laquelle est due l'occlusion de la fleur ou son sommeil. Ainsi, ces deux tissus incurvables quoique placés d'une manière inverse chez les mirabilis et chez le pissenlit, so courbent dans le même sens, c'est-à-dire que le tissu incurvable par endosmose se courbe en dehors et que le tissu incurvable par oxygénation se courbe en dedans, pour opérer l'un le réveil l'autro lo 
sommeil de la fleur. On sent quẻ l'extrême exiguïté de l'épaisseur do ces tissus incurvables chez le pissenlit, ne permet pas de les séparer l'un de l'autre pour expérimenter directement quel est le sens de l'incurvation propre à chacun d'eux, mais l'analogie no permet pas ici d'avoir des doutes à cet égard. L'expérience, d'ailleurs, prouve directement que le réveil est dû à la turgescence d'eau ou à l'endosmose implétive, et que le sommeil est dû à l'oxygénation. In effet, le demi-fleuron de la fleur du pissenlit étant cueilli de grand matin lorsqu'il a encore l'incurvation du sommeil (figure 12), et étant plongé dans l'eau aérée, il y prend de suite l'incurvation contraire (figure 11) qui est cello du réveil. Si on le plonge dans de l'eau non aérée, il $\mathrm{y}$ prend une courbure bien plus profonde (figure 13) qui est l'exagération de la courbure du réveil normal, et il y conserve constamment cette courbure. Si l'on transporte ces demi-fleurons ainsi courbés en dehors dans du sirop de sucre, ils se courbent en dedans (figure 12). Ainsi, il n'y a pas de doute que l'incurvation en dehors, qui opère lo réveil, ne soit due à l'endosmose implétive. Si on laisse séjourner pendant quelques heures le demi-fleuron qui est à l'état de réveil (figure 11) dans l'eau aérée, il y prend l'incurvâtion en dedans (figure 12) qui est celle de l'état de sommeil, et cette incurvation n'est point détruite en transportant le demi-fleuron ainsi courbé dans du sirop, ce qui prouve bien que cette incurvation secondaire n'est point due à l'endosmose. Comme cette incurvation secondaire n'a point lieu dans l'eau non aérée, cela prouvo qu'elle est 
due à l'oxygénation. Ainsi, le réveil et le sommeil des demi-fleurons de la fleur du pissenlit, résultent de l'incurvation alternativeınent prédominante d'un tissu organique incurvable par endosmose et d'un tissu organique incurvable par oxygénation. L'action actuelle de la lumière donne la supériorité de force d'incurvation au tissu cellulaire incurvable par endosmose, l'absence ou la diminution de la lumière laisse prédominer la force d'inourvation du tissu incurvable par oxygénation. Dans chacun de ces deux cas, le tissu incurvable qui a la supériorité d'action, entraìne malgré lui le tissu incurvable antagoniste dans le mode de flexion qui lui est propre. La supériorité d'action donnée le matin par la lumière au tissu incurvable par endosmose, provient de deux causes : $1^{\circ}$ de ce que sous l'influence de la lumière, la sève lymphatique afflue dans les demi-fleurons en plus grande abondance; ils attirent alors plus d'eau qu'ils n'en exhalent par la transpiration; $2^{\circ}$ de ce que le tissu incurvable par oxygénation a perdu pendant la nuit une partie de sa forco d'incurvation, ainsi que je vais le démontrer tout à l'heure. La supériorité d'action que prend le soir le tissu incurvable par oxygénation, provient aussi de deux causes : $1^{\circ}$ de la diminution de l'afflux de la sève lymphatique dans le tissu incurvable par endosmose; $2^{\circ}$ de l'augmentation d'oxygénation que subit pendant le jour le tissu iñcurvable par oxygénation. La diminution de l'afflux de l'eau dans les demi-fleurons pendant la nuit, se prouve par l'observation que j'ai faite que, durant les nuits chaudes et lorsque l'air est sec, les 
demi-fleurons dans leur état de sommeil ont perdu environ $1 / 25$ de la longueur qu'ils avaient pendant le jour précédent dans leur état de réveil. Ils sont sans doute aussi diminués de largeur, mais cela est insensible. Cetto diminution dans les dimensions, provient évidemment de ce que dans l'état de sommeil, pendant l'absence do la lumière, il y a diminution de l'afflux de la sève lymphatique qui rendait les cellules turgescentes. La diminution pendant la nuit de l'oxygénation du tissu incurvable par oxygénation résulte des expériences suivantes. Ayant ôté lo soir quelques demi-fleurons dans l'état de sommeil à une fleur de pissenlit qui devait le lendemain matin reprendre l'état de réveil, je les plongeai dans de l'eau aérée; ils y conservèrent invariablement leur incurvation de sommeil. Le tissu incurvable par oxygénation, à l'action duquel était due cette incurvation de sommeil, se courbait alors avec trop de force pour que son incurvation pût être vaincue par la teńdance à l'incurvation dans le sens opposé, qui devait être augmentée cependant alors par l'afflux de l'eau dans le tissu incurvable par endosmose. Le lendemain de grand matin, lorsque la fleur était encore pour plusieurs heures dans l'état de sommeil, je lui enlevai de nouveaux demifleurons et je les plongeai dans l'eau aérée. Ils ne tardèrent pas à perdre leur incurvation de sommeil, et ils prirent l'incurvation de réveil. Cette seconde expérience, pareillo à la première et cependant si différente par ses résultats, prouve que pendant la nuit le tissu incurvable par oxygénation à l'action duquel lo sommeil était dû, avait perdu 
une grande partie de sa force d'incurvation en dedans, puisqu'elle était alors vaincue promptement par l'action antagoniste du tissu incurvable par endosmose, lequel la veille au soir n'avait pu obtenir cette victoire. Il demeure prouvé par ces faits, que le tissu incurvable par oxygénation, acquiert de la force et par conséquent do l'oxygène pendant lo jour, et qu'il perd une portion de cette force et par conséquent une portion de son oxygène pendant la nuit. C'est très probablement alors en formant de l'acide carbonique, qu'il perd une partie de son oxygène acqquis sous l'influence de la lumière. Malgré cette perte nocturne d'une partie de l'oxygène acquis pendant le jour, il paraît que cette substance s'accumule de plus en plus dans le tissu incurvable par oxygénation; car lorsque le dernier sommeil arrive lo troisième jour de la vie de la fleur du pissenlit, il offre une incurvation des demi-fleurons beaucoup plus profonde que celle que présentait leur sommeil des deux jours précédents, ce qui me parait être le résultat de l'oxygénation excessive du tissu qui se courbe par implétion d'oxygène fixé. L'absence de la lumière n'occasionne donc pas l'élimination complète do l'oxygène fixé dans ce tissu pendant le jour, et il résulte do là l'accumulation progressive de cette substance dans le tissu organique qu'elle encombre, co qui constitue son état de vieillesse et amène sa mort. Cette théorie me paraît d'autant plus plausible, qu'elle est complètement d'accord avec des faits du même genre que j'ai exposés dans un autre Mémoire ${ }^{(1)}$.

(1) De l'usage physiologique de l'oxygène, considéré dans ses rapports avec l'action des excitants (dans le t. II). 
J'ai rapporté au commencement de ce mémoire les expériences que j'ai publiées en 1831, et qui m'ont fait voir que les fleurs placées dans le vide de la pompe pneumatique y conservent invariablement celui des deux états de réveil ou de sommeil qu'elles possèdent au moment où elles y sont placéris. La conservation de l'état do réveil par les fleurs placées dans le vide est une conséquence nécessaire de la paralysie du tissu fibreux incurvable par oxygénation, et agent du sommeil, paralysie occasionnée par la soustraction de l'air respirable; quant à la conservation de l'état de sommeil par les fleurs placées dans le vide lorsqu'elles sont dans cet état, cela provient du défaut d'afflux de la sèvo lymphatique dans lo tissu cellulaire incurvable par endosmose et agent du réveil. Les plantes fleuries qui ont été soumises à ces expériences trempaient dans l'eau par l'extrémité inférieure de leurs tiges, en sorto que l'eau ou la sève lymphatique ne pouvait parvenir aux fleurs que par l'attraction qu'elles exercent sur ce liquide dans l'état naturel. Or, j'ai prouvé dans un autre mémoire (1) que l'ascension de la sève lymphatique par attraction cesse d'avoir lieu lorsque la respiration végétale est.suspendue; or, la respiration des fleurs est complètement suspendue dans le vide; elles ne peuvent done plus attirer la sève lymphatique, dont l'aflux est indispensable pour occasionner la turgescence par endosmose, et par suite l'incurvation de leur tissu cellulaire agent du réveil : voilà pourquoi leur sommeil

(1) Voyez le Mémoire VIIT, p. 413. [Recherches sur les conçuits de la sève et sur les causcs de sa progression.] 
persiste dans le vide lorsuu'elles y sont placées dans çt état. Au reste, je dois dire que j'ai fait ces expériences sur des fleurs cueillies le soir lorsqu'elles venaient de prendre l'état de sommeil, état que l'incurvation du tissu cellulaire par endosmose ne peut faire cesser alors, ainsi que je l'ai fait voir plus haut. Le réveil est véritablement impossible le soir, en raison de la trop forte oxygénation du tissu fibreux agent du sommeil : peut-être le réveil d'une fleur aurait-il lieu dans le vide si elle y était placée le matin et récemment cueillie, car alors le réveil est devenu plus facile en raison de la désoxygénation partielle du tissu fibreux pendant la nuit. Le réveil de la fleur serait probablement alors encore plus facile si la plante à laquelle elle appartient tenait au sol par ses racines, par l'impulsion desquelles elle recevrait la sève lymphatique ascendante. Ce sont des expériences à faire ${ }^{(1)}$.

\section{§ II. - DU réveil ET DU SOMMIEIL DES FEUILLES.}

Bonnet est lo premier qui ait tenté d'expliquer les phénomènes du réveil et du sommeil des feuilles ${ }^{(2)}$; fondé sur cette hypothèse erronée de Dodart, que le tissu des racines se contracte par l'humidité, et que le tissu des tiges se contracte par la sécheresse, et que c'est à cela que l'on doit attribuer la descente

(1) Je n'avais point de machine pneumatique à ma disposition à la campagne, où j'ai fait la plupart des olservations et des expériences contenues dans ce Mémoire. Celles des expériences que j'y rapporte, et qui sont faites dans le vide, sont déjà anciennes : quelques-unes ont été publiées, d'autres étaient restées inédites.

(2) Recherches sur l'usage des feuilles. 
des racines et l'ascension des tiges, Bonnet crut pouvoir expliquer la position rclevée que les folioles du robinia pseudo-acacia affectent pendant lo jour et la position abaissée qu'elles présentent pendant la nuit, à ce que la face supérieure de ces folioles se contracterait par l'effet de la sécheresse pendant lo jour, tandis que leur face inférieure so contracterait par l'effet de l'humidité du soir. Il crut prouver cette assertion par une expérience assez étrange : il construisit des feuilles artificielles, leur face supérieure était en parchemin, dont lo tissu se resserro par l'effet de la sécheresse, et leur face inférieure était en toile, dont le tissu se resserre par l'effet de l'humidité; présentant ensuite ces feuilles artificielles à l'action successive d'une forte chaleur et de l'humidité, il leur fit exécutcr des mouvements qu'il crut comparables à celles que les folioles de l'acacia exécutent pour prendre leurs positions de réveil et de sommeil. Cette expérience et l'hypothèse qu'elle devait appuyer ont autrefois trouvé des admirateurs; aujourd'hui clles sont avec juste raison réléguées parmi les nombreuses aberrations de l'esprit humain. M. de Candolle(1) a envisagé sous leur vrai point de vue les phénomènes du réreil et du sommeil des feuilles, en reconnaissant qu'ils dépendent essentiellement do l'inflüence do la lumière. Il est parvenu à intervertir les époques habituelles du réveil et du sommeil chez des sensitives éclairées artificiellement pendant la nuit et mises à l'obscurité pendant le jour.

(1) Mémoire sur l'influence de la lumière artificielle sur les plantes; dans les Mémoires des savants étrangers de l'Institut, t. I. 
Les mouvements par lesquels les feuilles prennent les positions alternatives de réveil et de sommeil sont exécutés par certains renflements qui chez les feuilles composées sont situés à la base du pétiole commun, à la base de chaque pinnule, et qui composent en entier le court pétiole particulier de chaque foliole. Chez les feuilles simples qui ont un réveil et un sommeil, il y a un renflement moteur à la base du pétiole, il $\mathrm{y}$ en a un autre à son sommet, ou plutôt à la base de la nervure médiane do la feuille ou des nervures qui convergent au sommet du pétiole. Dans co cas, le limbe do la fenille exécuto des mouvements de réveil et de sommeil sur lo pétiole. Ces divers mouvements sont dus à l'incurvation et quelqucfois à la torsion des parties organiques qui entrent dans la composition des renflements moteurs. Ainsi c'est par incurvation que les feuilles et les folioles de la sensitive, du haricot, etc., prennent les positions de réveil et de sommeil; les folioles de la feuille des casses joignent la torsion à l'incurvation de leurs renflements moteurs pour prendro la position de sommeil. Cetto double action fait qu'en dirigeant leurs sommets vers la terre, les folioles opposées s'arpliquent les unes sur les autres par leurs faces supérieures.

Les phénomènes du réveil et du sommeil, si fugaces chez les fleurs dont la vié est de courte durée, s'observent pendant longtemps chez les feuilles, puisque la durée de leur vie est de plusieur's mois.

Lo haricot est, parmi les plantes indigènes, celle dont la feuillo offre le plus de facilité pour l'étude do ces phénomènes, à raison do la grosseur des 
renftements moteurs qui constituent à eux seuls le court pétiolo particulier des folioles de cette feuille, folioles dont le réveil et le sommeil sont si remarquables. Dans lo sommeil, les folioles du haricot dirigent leur pointe ou leur sommet vers la terre : le plan de la foliole est alor's vertical; dans lo réveil, le plan des folioles redevient horizontal. Cependant cela n'a pas toujours lieu, parce que les folioles ayant une nutation très marquée, elles tendent toujours à dipiger leur face supérieure vers le soleil lor'squ'il n'est point voilé par des nuages.

C'est exclusivement sur lo renflement moteur ou sur le pétiole des folioles qu'agit la lumière pour déterminer le réveil par sa présence et le sommeil par son alusence; son action sur le limbe de la feuille est ou paraît être nulle pour cet objet, ainsi que le prouve l'expérience suivanto : j'ai enlevé toute la partie membraneuse des folioles d'une feuille do haricot, en laissant subsister une portion de leur nervure médiane, afin de pouvoir observer leurs mouvements d'élévation ou d'abaissement; ces mouvements qui constituent, le premier le réveil et le second le sommeil, continuèrent d'avoir lieu; j'ai observé de même qu'une feuille de sensitive étant dépouillée de ses folioles, le pétiole continue de présenter ses mouvements d'abaissement ou de sommeil, et d'élévation ou do réveil. Il est donc incontestable que co sont les pétioles, ou plutôt leurs renflements moteurs, qui seuls éprouvent do la part de la lumière l'influence qui les détermine à prendre les incurvations qui constituent lo sommeil et lo réveil. 
Pour étudier la structure intérieure du renflement moteur de chaque foliole de la féuille du haricot, j'ai soumis au microscope des tranches minces enlevées transversalement et longitudinalement sur ces renflements moteurs. La figure 15 représente leur

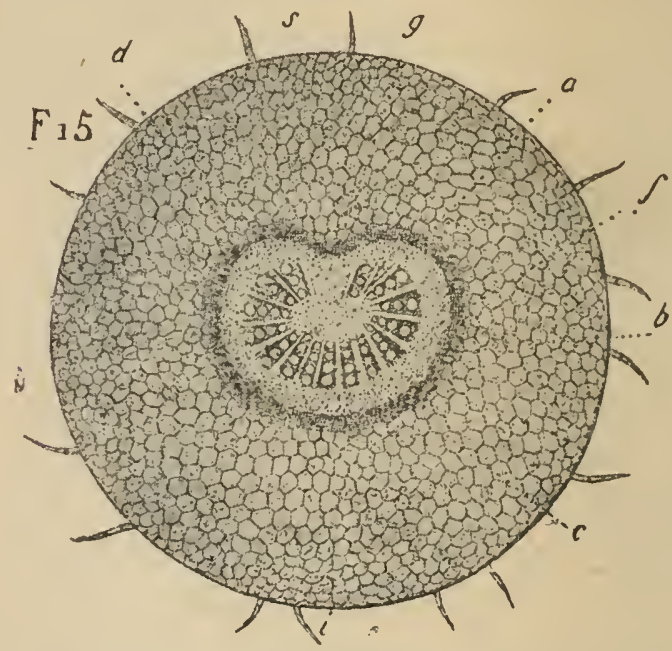

Fig. 15. - Coupe transversale du renflement moteur d'une foliole de la feuille de haricot. $s$, côté supérieur; $i$, côté inférieur; $c$, tissu -ellulaire incurvable par turgescence de liquide et composé de cellules qui décroissent principalement de grandeur du dedans vers le dehors, ce qui fait que l'incurvation de ce tissu cellulaire tend à s'opérer vers le dehors. $b$, cellules pneumatiques. $f$, couche de tissu fibreux incurvable par oxygénation. $d$, trachées remplies d'air et dont les faisceaux sont séparés par des rayons qui partent du centre $a$, lequel est occupé par du tissu fibreux semblable à oelui de la couche $f$.

coupe transversale. On voit au-dessous de l'enveloppe tégumentaire une couche épaisse de cellules $c$ dont les parois offrent une multitude de globules ccllulaires. Ces cellules augmentent insensiblement de grandeur en allant vers le centre. Cet accroissement de grandeur des cellules s'arrête à 
peu près aux trois quarts do l'épaisseur de cetto couche cellulaire : c'est là que se trouve les plus grandes cellules. A partir de cet endroit, les cellules vont en décroissant de grandeur vers le centre; les plus internes $b$ sont remplies d'air; elles se dessinent en un cercle blanc sur la coupe transversale du renflement moteur observée à la loupe et à la lumière réfléchie; elles paraissent noires parce qu'elles sont opaques lorsqu'on observe au microscope et par transparence une tranche mince et transversale du renflement moteur. C'est à ces caractères que l'on reconnaît le tissu cellulaire rempli d'air, et qu'on le distingue du tissu cellulaire rempli de liquides. Co dernier, observé par transparence, est toujours plus ou moins diaphane. Telle est la couche épaisse de tissu cellulaire $c$ qui recouvre les cellules pneumatiques $d$. Ce tissu cellulaire offrant dans la majeure partio de sa masse lo décroissement de ses cellules du dedans vers le dehors, doit avoir pour action générale de se courber de manière à diriger la concavité de sa courbure vers le dehors lorsqu'il devient turgescent. C'est aussi ce que l'expérience démontre, car en plongeant dans l'eau une lame mince enlevée longitudinalement sur ce tissu cellulaire, elle se courbe fortement dans le sens que je viens d'indiquer. Si l'on transporte dans du sirop cette lame ainsi courbée, elle se courbe en sens inverse. Ainsi ce tissu cellulairo est incurvable par endosmose. Il représente par sa disposition un cylindre creux dont toutes les parties longitudinales, si elles étaient séparées les unes des autres, tendraient dans l'état naturel à se courber 
vers le dehors. Au-dessous des cellules pneumatiques $b$ est une couche de tissu fibreux $f$ souvent recourbée sur elle-même en demi-cercle dont les extrémités sont jointes imparfaitement on g. Ce tissu fibreux est tout à fait semblable à celui dont j'ai plus haut noté l'existence chez les fleurs qui offrent les phénomènes du sommeil et du réveil; mais il est ici beaucoup plus facile à observer, tant dans sa masse que dans sa structure intime. Une lame enlevée longitudinalement sur ce tissu fibreux situé sous le tissu cellulaire, étant plongée dans l'eau aéróe, elle s'y courbe en dirigeant la concavité de sa courbure vers le centre du pétiole. Si cette lame est plongée dans l'eau non aérée, ello ne se courbe point du tout. Ainsi ce tissu fibreux est incurvable par oxygénation; il représente par sa disposition un cylindre creux dont toutes les parties longitudinales, si elles étaient séparées les unes des autres, tendraient dans l'état naturel, à se courber vers le dedans ou vers le centre du pétiole. Au-desisous de cette couche du tissu fibreux $f$ se trouve un corps ligneux rayonné composé de tubes séveux et de gros tubes pneumatiques dont on voit ici les ouvertures. Des rayons médullaires divergents traversent ce corps ligneux; ils paraissent contenir du tissu fibreux semblable à celui de la couche $f$; ils partent d'une partie centrale a qui est oceupée par un faisceau de tissus fibreux exactement semblable, par son aspect et par son organisation, au tissu fibreux de la couche $f$. On reconnaît ce tissu aux ponctuations noires sur un fond transparent qu'il offre sur sa coupe transversale observée au microscope par 
transparence. Il m'a scmblé que ces points opaques étaient les ouvertures de tubes pneumatiques d'une excessivo petitesse dont lo tissu fibreux $f$ et $a$ serait pénétré dans toute sa masse. Il est facile de voir que le pétiole représente ici un segment longitudinal de tige, ainsi que je l'ai déjà établi ailleurs (1). Le tissu cellulaire $c$ et $b$ appartient au système cortical dont il représente le parenchyme auquel j'ai donné le nom de médule corticale. Le corps ligneux rayonné $d$ est la moitié de la partie ligneuse du système central avec ses rayons médullaires; le tissu fibreux $a$, qui est au centre, et duquel partent les rayons médullaires, représente la moitié de la moelle ou médule centrale, laquelle est ici métamorphosée en tissu fibreux incurvable par oxygénation. Les rayons médullaires s'étendent de ce tissu fibreux $"$ au tissu semblable de la couche $f$. La petitesse du faisceau central du tissu fibreux $a$, m'a empêché de m'assurer par l'expérience du sens de son incurvation, sens qui peut, je pense, être déterminé rationnellement. Ce tissu fibreux central $a$ est une métamorphose de la moelle dont il occupe la place, et il ne représente que la moitié longitudinale de cette moelle. Or la moelle, généralement composée de cellules qui décroissent de grandeur du centre vers la circonférence, tend par cela même, lorsqu'elle est turgescente, à courber: ses deux moitiés longitudinales séparées en dirigeant leur concavité ver's le dehors. Le faisceau central de tissu fibreux $a$ qui représente une des moitiés longitudinales de la

(1) Voyez le Mémoire III, p. 200. [Recherches sur l'accroissement des vegétaux, in Mémoires, t. 1.7 
moelle dont il est une métamorphose, doit donc avoir le même sens d'incurvation que cette moelle si elle existait dans son état normal, c'est-à-dire qu'il doit se courber de manière à diriger la concavité de sa courbure vers le côté $i$, qui est pour lui le véritable côté de dehors de la tige dont le pétiole représente seulement la moitié longitudinale, le côté $s$ est

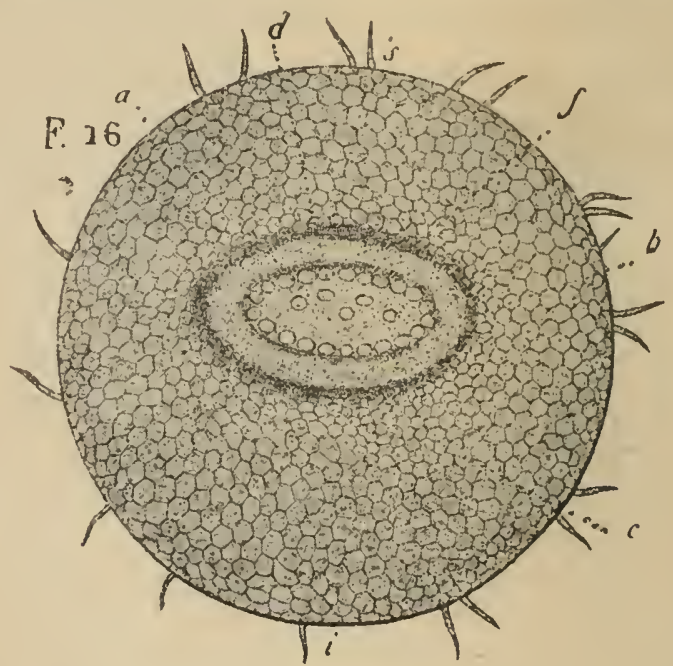

FIg. 16. - Coupe transversale du renflement moteur du pétiole de la feuille de sensitive. $\delta$, côté supérieur; $i$, côté inférieur; $c$, tissu cellulaire incurvable par turgescence de liquide et composé de cellules qui décroissent principalement de grandeur du dehors vers le dedans, ce qui fait que l'incurvation de ce tissu cellulaire tend à s'opérer vers le dedans. $b$, cellules pneumatiques; $f$, couche de tissu fibreux incurvable par oxygénation; $d$, tubes pneumatiques; $a$, faisceau central de tissu fibreux semblable à celui de la couche $f$, et inêlé de quelques tubes pneumatiques.

véritablement le côté de dedans de cette moitié longitudinale de tige. Comme le tissu fibreux de la la couche $f$ tend à so courber en dirigeant sa concavité vers le centre du pétiole ou du ronflement moteur, il en résulto quo le faisceau central de tissu 
fibreux $a$ est, par le sens de son incurvation, congénère de la partie supérieure de la couche de tissu fibreux $f$, couche qui est ici un peu déprimée en $g$ et qui quelquefois est interrompue dans cet endroit. Cette dépression, indice d'une séparation primitive, disparaît tout à fait dans le renflement moteur du pétiole de la sensitive, comme on peut le voir dans la figure 16. Le tissu fibreux de la couche $f$ tendant à se courber en dedans, sens d'incurvation qui est celui des couches internes du système cortical, je

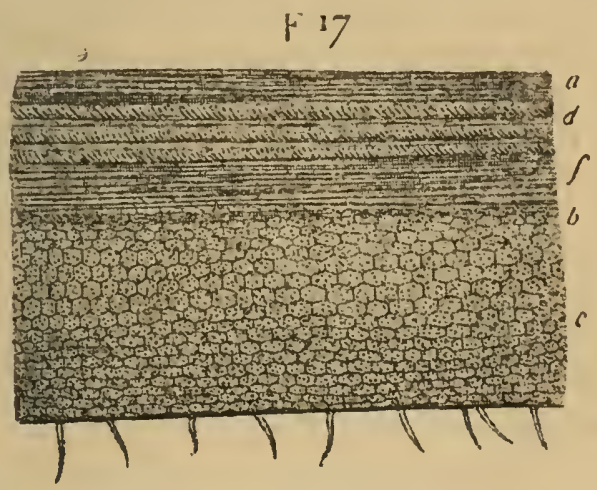

Fig. 17. - Coupe longitudinale de la moitié inférieure du renflement moteur de la foliole du haricot. Les mêmes lettres indiquent les mêmes objets dans cette figure et dans la figure $\mathbf{1 5}$.

suis porté à penser qu'il est produit par une inétamorphose du liber fibreux de l'écorce; peut-être même, et c'est ce qui me paraît le plus probable, est-il ce liber fibreux lui-même à l'état naissant, état dans lequel il aurait des propriétés qui deviennent étrangères au tissu fibreux de l'écorce lorsqu'il a vieilli. La figure 17 représente la coupe longitudinale et médiane de la moitié inférieure du renflement moteur de la foliole du haricot, renflement 
moteur dont je viens de montrer la coupe transversale. Les mêmes lettres indiquent les mêmes objets que dans la figure 15. Les tubes pneumatiques $d$ qui avoisinent en dedans la couche de tissu fibreux $f$, sont des grosses trachées qui sont tellement couvertes de globules, qu'on les prendrait facilement pour des tubes ponctués si elles no se déroulaient pas. J'ai pu observer avec assez de facilité l'organisation intime du tissu fibreux $f$ et $a$, en soumettant au microscope des lames extrêmement minces enlevées longitudinalement sur ce tissu. Je n'avais pas pu voir cette organisation d'une manière aussi claire chez les fleurs que j'ai étudiées plus haut. Le tissu fibreux $f$ offre deux éléments qui sont associés et mêlés, savoir : des fibres longitudinales, transparentes, d'une finesse extrême, et des globules cellulaires extrêmement petits, placés à la file et formant ainsi des séries longitudinales. Les fibres sont disposées par faisceaux, dans les intervalles desquels existent les séries de globules cellulaires. Ces globules sont semblables à ceux que l'on voit sur les parois des cellules de la couche corticale $c$ et sur les parois des trachées $\iota$. Leur nombre prodigieux dans toutes les parties qui jouissent éminemment de l'excitabilité prouve, ce me semble, que c'est à l'exercice de cette fonction qu'ils sont destinés. Les fibres de la couche $f$ ne sont point partout de la même grośseur; j'ai vu que dans cette couche $f$ (figure 17) les fibres les plus grosses sont voisines des cellules pneumatiques $b$, elles ont $1 / 300$ de millimètre de diamètre. Les fibres les plus petites sont voisines des trachées $d$; je leur ai trouvé environ 
1/500 de millimètre de diamètre. Cette grosseur décroissante du dehors vers le dedans, est évidemment la cause qui fait que lors de l'implétion de leur cavité, ces fibres qui paraissent tubuleuses courbent en derlans le tissu qu'elles forment par leur assemblage. La substance qui opère cette implétion est ici l'oxygène, aussi la couche $f$ de ce tissu incurvable par oxygénation, est-elle compriso entre deux couches d'organes remplis d'air, savoir : en dehors les cellules pneumatiques $b$ et en dedans les trachées $d$. J'ai fait voir plus haut, que la même disposition existe chez les fleurs qui possèdent co même tissu incurvable par oxygénation. La manière dont se fait cette implétion d'oxygène ou cette turgescence des fibres tubuleuses par oxygénation me semble facile à comprendre. Admettant que dans ces fibres tubuleuses il existe un liquide qui a beaucoup d'affinité pour l'oxygène, l'addition de cette substance à ce liquide en allgmentera nécessairement la masse et produira, par conséquent, la turgescence de ces fibres tubuleuses. Dès lors, l'incurvation du tissu qu'ellés forment par leur assemblage, s'opérera de manière que les fibres les plus petites seront à la concavité de la courbure et les fibres les plus grosses à sa convexité.

Les renflements moteurs des folioles de la feuille du robinia pseudo-acacia offrent assez exactement la même organisation que celle qui vient d'être exposée pour le haricot, et les phénomènes d'incurvation par endosmose et par oxygénation que présentent leurs tissus, sont les mêmes. Aussi ces deux plantes offrent-elles de même le réveil en élevant 
leurs folioles et le sommeil en les abaissant. Or, chez d'autres plantes c'est l'inverse qui a lieu : ainsi dans la feuille de la sensitive (mimosa pudica L.), les folioles sont redressées dans l'état de sommeil et leur plan redevient horizontal dans l'état de réveil. Il n'en est pas de même par rapport au pétiole de la feuille de cette même plante : il s'abaisse dans lo sommeil et il se relève dans le réveil, comme cela a lieu pour les folioles de la feuille du haricot. Le renfiement moteur situé à la base de ce pétiole et agent de ses mouvements, offre seul assez de grosseur pour pouvoir être soumis à l'observation et à l'expérience. Les renflements moteurs des pinnules et des folioles de cette feuille sont trop petits pour ce double objet; ainsi, j'ai dû me borner à l'étude de la structure du renflement moteur du pétiole et à l'observation des phénomènes de mouvement qu'il présente. Ce renflement moteur, représenté un peu grossi dans la figure 18, est droit $a b$ lorsque la feuille est dans l'état de réveil; alors le pétiole est redressé; ce même renflement moteur est courbé vers le bas $c$, lorsque la feuille est dans l'état de sommeil; alors le pétiole est abaissé. Il semblerait que les phénomènes du réveil et du sommeil étant ici les mêmes que ceux que l'on observe dans les folioles du haricot, la structure intérieure du renflement moteur devrait être semblable. Or, l'observation contredit en un point cette induction de l'analogie. J'ai dit, en décrivant le renflement moteur de la foliole de haricot, que son tissu cellulaire $c$ (fig. 15 et 17) offre deux sens de décroissement de grandeur de ses cellules, savoir : un décroissement prédo- 
minant du dedans ver's le dehors, et un faible décroissement dirigé vers le centre. Il résulte do cette structuro, que co tissu cellulaire tend généralement à se courber ver's le dehors. Or, l'observation apprend que ees deux couches cellulaires à décroisse-

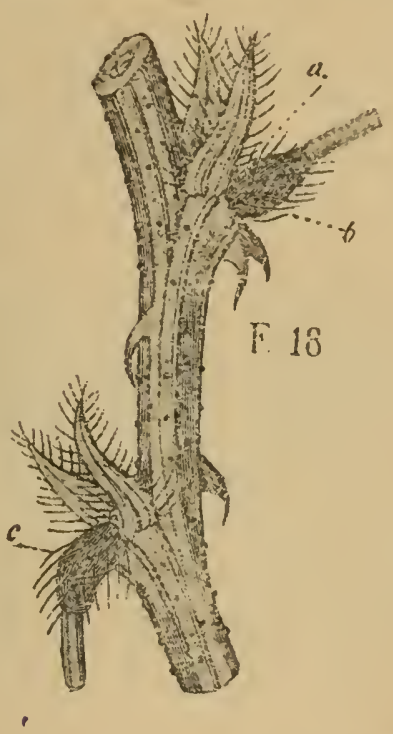

FrG. 18. - Portion de tige de sensitive portant deux feuilles dont on ne voit ici que la partie inférieure des pétioles occupée par les renflements moteur's $a$ et $c$. En $a$, le renflement moteur est droit et le pétiole est redressé, ce qui constitue l'état de réveil; en $c$, le renflement moteur est courbé vers la terre, ce qui dirige le pétiole dans le méme sens : c'est l'état de sommeil.

ment inverse existent aussi dans le renflement moteur du pétiole de la sensitive, mais ici c'est la couche cellulaire dont le décroissement de grandeur a lieu du dehors vers le centre qui est prédominante; la couche cellulaire dont le décroissement des cellules est inverse, est presque réduite à rien, ainsi qu'on le voit dans la figure 16 qui représente 
la coupe transversale du renflement moteur du pétiole de la sensitive. La figure 19 représento la coupo longitudinale de la moitié inférieure de ce même renflement moteur. On voit d'abord sous l'enveloppe tégumentaire une couche épaisse de tissu cellulaire $c$, dont les cellules offrent leur décroissement de grandeur prédominart du dehors vers le dedans; il n'y a qu'une couche fort mince des cellules les plus superficielles qui décroissent de grandeur du dedans vers le dehors. On a vu plus haut, que

\section{F. 19}

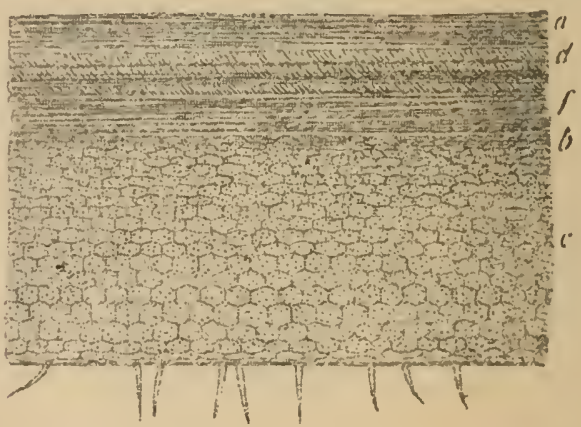

Frg. 19. - Coupe longitudinale de la moitié inférieure du renflement moteur du pétiole de la feuille de sensitive. Thes mêmes lettres indiquent les mêmes objets dans cette figure et dans la figure 16.

c'est cette dernière couche cellulaire, ici insignifiante, qui prédomine dans le renflement moteur des folioles du haricot. Le sens de l'incurvation du tissu cellulaire dans le renflement moteur du pétiole chez la sensitive, doit donc être inverse de celui qui a lieu dans ce mêmo tissu dans le renflement moteur des folioles chez le haricot. C'est aussi ce que l'expérience démontre. Une lame mince enlevée longitudinalement sur le tissu cellulaire du renflement 
moteur chez la sensitive étant plongée dans l'eau, elle se courbe fortement de manière à diriger la concavité de sa courbure vers l'axe du pétiole. Si on la transporte dans du sirop de sucre, elle se retourne et se courbe en sens inverse. Ainsi, ce tissu cellulaire est incurvable par endosmose. Dans l'ordre naturel, c'est l'accession de la sève lymphatique arrivant à l'extérieur des cellules par les méats intercellulaires qui détermine leur implétion par endosmose; car elles contiennent un liquide dense qui est coagulable par la chaleur, par les acides et par l'alcool (1), leurs parois offrent une grande quantité de globules verts. La couche la pluis intérieure $b$ de ce tissu cellulaire ne contient que de l'air dans ses oellules. Au-dessous de ces cellules pneumatiques, se trouve une couche de tissu fibreux $f$; son aspect et son organisation sont entièrement semblables au tissu fibreux incurvable par oxygénation qui occupe la même place dans le renflement moteur do la foliole de haricot. J'aurais voulu m'assurer par une expérience directe, que ce tissu ne se courbait point dans l'eau non aérée, mais jo n'ai pu faire cette expérience, parce que chez la sensitive ce tissu fibreux enlevé en une lame mince, n'attend pas qu'il soit plongé dans l'eau aérée pour se courber, il se courbe dans l'air à l'instant même où il est enlevé, en sorte quo jo

(1) J'ai autrefois représenté le tissu du renflement moteur du pétiole de la sensitive comme contenant un grand nombre de corps globuleux opaques. Cette fausse apparence provenait de ce que chaque cellule contenait un coagulum globuleux produit par l'action de l'aoide nitrique que j'employais pour dissocier les organes élémentaires des tissus organiques. 
n'ai pu voir si son incurvation refuserait de s'opérer en le plongeant dans l'eau non aérée; car cette incurvation une fois prise ne se perd point même dans l'eau non aérée. Au reste, j'ai vu que le sens do l'incurvation de ce tissu fibreux est le même que chez le haricot, c'est-à-dire que la concavité de sa courbure est dirigée vers lo centre du pétiole. Audessous de ce tissu fibreux, qui bien certainement est incurvable par oxygénation, se trouve une couche ligneuse fort minoe reconnaissable à son opacité, ce sont là les tubes séveux qui sont mêlés à de nombreux tubes pneumatiques dont on voit ici les ouvertures en $d_{\text {s. }}$ Au centre enfin, et en remplacement de la moelle se trouve un faisceau de tissu fibreux $a$, entièrement semblable par son organisation au tissu fibreux de la couche $f$, c'est donc aussi du tissu fibreux incurvable par oxygénation; il possèdo dans son milieu des trachées qui sont remplies d'air et dont on voit ici les ouvertures. La coupe longitudinale de la moitié inférieure de ce renfiement moteur, est représentéo par la figuro 19; les mêmes lettres indiquent les mêmes objets. On y voit que les cellules du tissu cellulaire sont disposées en séries longitudinales, de la même manière que cela a lieu dans le renfiement moteur de la foliole de haricot (figure 17). Je ferai remarquer que le tissu cellulaire do la partio inférieure $i$ (figure 16), est plus épais que celui de la partie supérieure $s$. Le rapport das épaisseurs de ces deux parties du tissu cellulaire est à peu près celui de 5 à 3 .

Je viens d'exposer la structure de deux renflements moteurs qui, tous deux, opèrent le réveil par 
lo redressement des parties qu'ils meuvent et qui opèrent le sommeil par l'abaissement de ces mêmes parties. Il me fallait encore étudier la structure d'un renflement moteur qui agît dans un sens inverse, c'est-à-dire qui opérât le réveil par l'abaissement et le sommeil par l'élévation des parties qu'il est chargé de mouvoir; il fallait en outre que

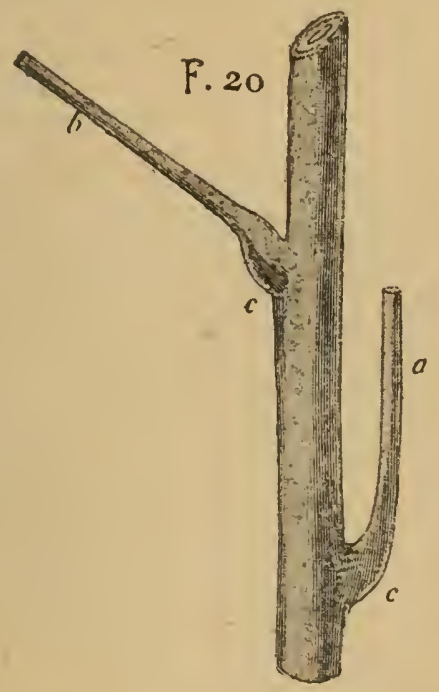

Fig. 20. - Portion de tige d'hedysarum stroliliferum, portant deux feuilles, dont on ne voit ici que la portion inférieure des pétioles, à la base de chacun desquels existe un renflement moteur $c, c$. Le pétiole $b$ est dans l'etat de réveil, le pétiole $a$ est dans l'état de soinmeil.

co renflement moteur fût assez gros, pour pouvoir être étudié dans sa structure. J'ai trouvé toutes ces conditions réunies chez l'hedysarum strobiliferum L. Le pétiole de la feuille de cette plante prend la position de sommeil en se relevant, jusquà toucher presque la partie de la tige située au-dessus de lui (figure 20,a). Dans sa position de réveil, il s'abaisse 
en s'éloignant de la tige jusqu'à ce qu'il fasse avec elle un angle de 50 à 60 degrés, $b$; le renflement moteur $c$, agent de ces mouvements est, comme à l'ordinaire, situé à la base du pétiole. Le limbe, ici absent, de la feuille simple que porte co pétiole, est mû par un autre renflement moteur situé au sommet de ce pétiole; il abaisse le limbe de la feuille dans le sommeil et il le relève dans le réveil. Comme ces derniers phénomènes sont semblables à ceux que

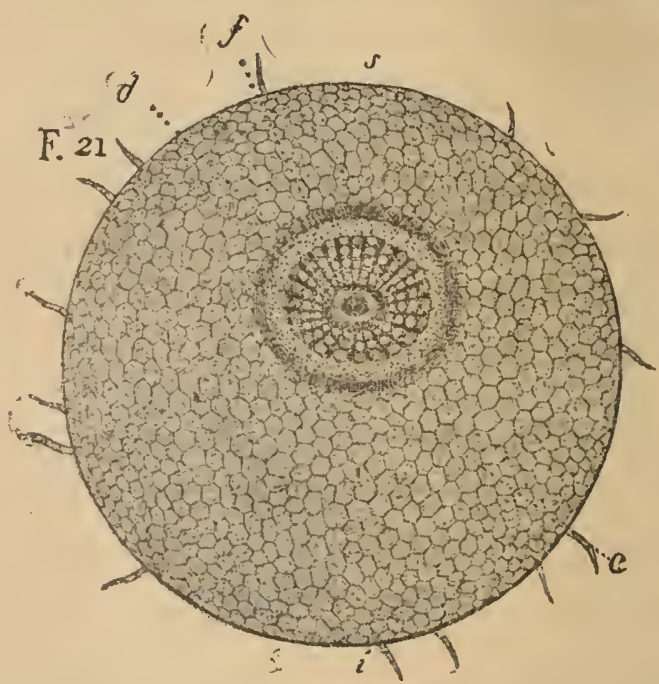

Fie. 21. - Coupe transversale du renflement moteur du pétiole de la feuille de l'hedysarum strobiliferum. $s$, côté supérieur; $i$, côté inférieur. $c$, tissu cellulaire incurvable par turgescence de liquide et composé de cellules qui décroissent principalement de grandeur du dedans vers le dehors, ce qui fait que l'incurvation de ce tissu cellulaire tend à s'opérer vers le dehors. $f$, couche de tissu fibreux incurvable par oxygénation. $d$, tubes pneumatiques dont les faisceaux sont séparés par des rayons partant du centre.

présentent les folioles du haricot, je ne m'en occuperai pas; je fixerai l'attention seulement sur le renflement moteur $c$ qui meut le pétiole de la feuille. La figure 21 représente la coupe transversale de co 
renflement moteur. On y voit les mêmes tissus qui existent dans tous les autres renflements moteurs, savoir : $1^{\circ}$ un tissu cellulaire $c$ incurvable par endosmose et tendant ici à se courber vers le dehors parce que ses cellules décroissent principalement du dedans vers le dehors; $2^{\circ}$ un tissu fibreux $f$ incurvable par oxygénation et tendant à se courber vers le dedans; $3^{\circ}$ un tissu ligneux rayonné, dans lequel existent beaucoup de tubes pneumatiques $d$. On remarquera qu'ici l'axe du pétiole est très excentrique, en sorte que la couche de tissu cellulaire $c$ est deux fois plus épaisse au côté inférieur ou externe $i$ qu'au côté supérieur ou interne $s$.

Ces faits anatomiques étant établis, il reste à déterminer comment agissent les deux tissus incurvables contenus dans les renflements moteurs, pour opérer les positions de réveil et de sommeil des feuilles et des folioles qu'ils meuvent.

Par une section longitudinale, j'ai retranché toute la moitié supérieure $a$ (fig. 18) du renflement moteur du pétiolo d'une feuille de sensitive; la moitié inférieure $b$ restée seule, s'est courbée de manière à diriger la concavité de sa courbure vers le haut, en sorte que le pétiole s'est trouvé dans un état de redressement exagéré, et cet état a persisté sans éprouver aucune variation. J'ai fait la contreépreuve : j'ai retranché toute la moitié inférieure $b$ du renflement moteur, en laissant subsister la moitié supérieure a. Cette dernière s'est fortement courbée de manière à diriger la concavité de la courbure vers le bas, ce qui a fortement abaissé le pétiole, et cet état d'abaissement a persisté invariablement. 
On pourrait conclure de ces expériences, ainsi que je l'ai fait autrefois, que c'est toute la moitié supérieure $a$, qui, par son incurvation vers le centre $\mathrm{du}$ renflement moteur, est l'agent de l'abaissement du pétiole ou de son sommeil, et que c'est toute la moitié inférieure $b$ qui, par son incurvation dirigée également vers le centre, opère par son antagonisme le redressement du pétiole ou son réveil. Le tissu cellulaire et le tissu fibreux, contenus dans chacune de ces deux moitiés du renflement moteur, tendent également à se courber vers le centre de ce renflement, en sorte qu'ici l'on serait porté à admettre que ces deux tissus incurvables coopèrent ensemble et au réveil et au sommeil, savoir : au premier par leurs parties contenues dans la moitié supérieure a du renflement moteur, et au second par leurs parties contenues dans la moitié inférieure $b$. Il faudrait alors admettre que ces deux moitiés, supérieure et inférieure, qui représentent deux ressorts antagonistes, reçoivent alternativement, le matin et le soir, un excès de force qui rend chacun de ces ressorts vainqueur du ressort opposé, lequel se trouve alors courbé dans un sens contraire à celui de la tendance naturelle à l'incurvation. C'est à cette théorie que je m'étais arrêté, lors de mes premières recherches sur le mécanisme des mouvements de la sensitive. $J_{\theta}$ pensais alors que ces mouvements reconnaissaient pour cause unique l'incurvation par endosmose du tissu cellulaire contenu dans le renflement moteur; ma découverte récente de l'existence, dans ce renflement moteur, du tissu fibreux incurvable par oxygénation, n'aurait pas changé peut-être ma théorie à 
cet égar'd, si je m’étais borné à l'étude des mouvements qui, chez le pétiole do la sensitive, produisent les positions de réveil et do sommeil. L'étudo de ces mêmes mouvements, dans les feuilles de plusieurs autres plantes, m'a fait voir quo cette théorie, néo d'une expérience trompeuse, devait être abandonnée. En effet, ayant fait sur les renflements moteurs des folioles du haricot les mêmes ablations que j'avais faites sur le renffement moteus du pétiole do la sensitive, j'obtins des résultats exactement semblables; l'ablation de la moitié longitudinale supérieure du renflement moteur fit que la moitié longitudinale inférieure restante se courba vers le haut et redressa la foliole qui demeura invariablement dans cet état de redressement qui est celui du réveil; l'ablation de la moitié longitudinale inférieuro du renflement moteur fit que la moitié longitudinale supérieure restante se courba vers le bas et abaissa la foliole qui derneura invariablement dans cet état d'abaissement. Or, en se rapportant à ce qui a été exposé plus haut touchant la structure inférieure du renflement moteur des folioles du haricot (fig. 15 et 17 ), on voit que son tissu cellulaire $c$ tend à se courber vers le dehors, tandis que son tissu fibreux $f$ tend à se courber vers le dedans ou vers le centre du pétiole; par conséquent, c'est ici le tissu fibreux $f$ qui est le seul agent de l'incurvation vers le dedans que prend une des moitiés longitudinales du renflement moteur, lorsqu'on a opéré l'ablation de la moitié longitudinale opposée; le tissu cellulaire est alors courbé de force dans un sens opposé à colui de sa tendance naturelle à l'in- 
curvation. D'après cela, ce serait ici le tissu fibreux de la couche circulaire $f$ (fig. 15) qui, par sa moitié supérieure, opérerait l'abaissement de la foliole ou son sommeil, et ce serait encore ce même tissu fibreux, qui par la moitié inférieure de sa couche circulaire, opérerait l'élévation de la foliole ou son réveil; le tissu cellulaire $c$ ne servirait point ainsi à l'exécution de ces mouvements. Cela est trop manifestement contraire à ce qui a été exposé plus haut relativement au mécanisme des mouvements auxquels sont dus le réveil et le sommeil des fleurs, pour qu'une semblable théorie puisse être fondée. $\checkmark$ 'ai fait voir, en effet, que sur les fleurs, le tissu cellulaire incurvable par endosmose est le seul agent du réveil, et que le tissu fibreux incurvable par oxygénation est le seul agent $d u$ sommeil. Il devient donc probable, par analogie, qu'il en est de même par rapport au réveil et au sommeil des feuilles, et l'expérience intervient pour changer cette probabilité en certitude.

J'ai fait voir qu'en privant d'oxygène le tissu fibreux incurvable par oxygénation, on paralysait ce tissu agent du sommeil ; en sorte que les fleurs soumises à cette expérience, conservaient invariablement l'état de réveil; c'est ce.qui a lieu lorsqu'on plonge une fleur, à l'état de réveil, dans l'eau non aérée privée de communication avec l'air atmosphérique. J'ai observé que les feuilles, susceptibles do réveil et do sommeil, continuent d'offrir ces mouvements alternatifs lorsqu'elles sont plongées dans l'eau aérée, mais ces mouvements éprouvent alors une certaino modification. Leur sommeil dans 
l'eau est aussi profond que dans l'air, c'est-à-dire que la flexion qui le constitue est aussi profonde, mais leur réveil est incomplet. Ainsi, par exemple, une feuille de robinia pseudo-acacia, plongée dans l'eau, offre le sommeil de la même manière que dans l'air, c'est-i-dire en appliquant ses folioles opposées l'une contre l'autre par leur face inférieure; mais le réveil qui, dans l'air, va jusquà amener les paires de folioles à la position diamétralement opposée, c'est-ì-dire jusqu'à leur faire décrire une demi-circonférence de cercle; le réveil, dis-je, de ces folioles plongées dans l'eau, ne va que jusqu'à leur faire décrire environ un huitième de circonférence de cercle, en sorte que leur mouvement de réveil dans l'eau n'est que lo quart de ce qu'il est dans l'air. Je ferai voir plus bas quelle est la cause de ce phénomène que je mo borne ici à exposer. Or, j'ai expérimenté qu'une feuille de robinia pseudoacacir étant plongéo dans l'eau non aérée sans communication avec l'air atmosphérique, ses folioles y prement et y conservent invariablement la position qui est celle de leur plus grand réveil dans l'eau aérée, leur sommeil n'a plus lieu. Or, comme il est démontré que l'immersion dans l'eau non aérée n'abolit l'incurvation que du seul tissu fibreux incurvable par oxygénation, il en résulte que c'est a ce seul tissu fibreux ici paralysé, qu'était dû le sommeil qui se trouve supprimé. Le réveil qui est aussi complet qu'il peut l'êrre dans l'eau, a done pour agent le seul tissu cellulaire incurvable par endosmose, lequel par son immersion dans l'eau même non aćrée se trouve dans une position favo- 
rable à l'exercice de l'endosmose qui doit rendre ses cellules turgescentes. Une autre expérienco d'un genre analogue faite sur une sensitive, m'a conduit aux mêmes résultats. Ayant placé une sensitive plantée dans un pot sous le récipient de la pompe pneumatique, ses feuilles se ployèrent dès le premier coup de piston, éprouvant, à ce qu il paraît, une excitation par la diminution de la densité de l'air. Lorsque lo vide fut achevé, les feuilles se relevèrent et so déployèrent, et elles restèrent invariablement dans cet état de réveil malgré l'obscurité de la nuit. Cette suppression $d u$ sommeil coïncidait encore ici avec l'abolition de l'action du tissu fibreux, lequel se trouvait paralysé par la soustraction de l'air respirable contenu dans les organes pneumatiques de la plante. Il est donc prouvé par ces expériences que, chez les feuilles comme chez les fleurs, le sommeil est dû à l'action du seul tissu fibreux incurvable par oxygénation, d'où il résulte nécessairement que le réveil est également dû chez les feuilles à l'action du seul tissu cellulaire incurvable par endosmose. Il ne s'agit donc plus actuellement que de rechercher comment ces deux tissus incurvables agissent séparément dans les renfiements moteur's des feuilles, pour produire leur réveil et leur sommeil.

Je prends pour premier exemple la feuille du haricot, dont les folioles sabaissent dans le sommeil et se relèvent dans le réveil. J'ai fait voir que dans le renflement moteur de ces folioles, les deux tissus incurvables représentent deux cylindres creux emboîtés l'un dans l'autre (fig. 15); le tissu cellulaire $c$ 
est en dehors et lo tissu fibreux $f$ est en dedans. Les deux couches cylindriques emboîtées l'une dans l'autre que forment ces deux tissus, seraient représentées assez exactement par la réunion et la soudure en cylindre creux d'un certain nombre de nervures de fleurs de mirabilis. J'ai fait voir que chez ces nervures, le tissu cellulaire tend à se courber ver's le dehor's par endosmose, et que le tissu fibreux tend à se courber vers le dedans par oxygénation, ce qui produit dans le premier cas la position de réveil de ces nervures et dans le second cas leur position de sommeil. Or, il en serait de même chez le renflement moteur de la foliole de haricot, si l'on supposait par la pensée que les deux couches cylindriques, l'une extérieure de tissu cellulaire, l'autre -intérieure de tissu fibreux, soient divisés à la fois en faisceaux longitudinaux. Chacun de ces faisceaux serait analogue à une nervure do fleur de mirabilis; il aurait en lui, et disposés comme dans cette nervure, les deux tissus incurvables capables d'opérer le réveil et le sommeil. Si l'on supposait entre ces faisceaux un tissu membraneux, cela formerait une corolle susceptible tour à tour d'épanouissement et d'occlusion, ou de réveil et de sommeil. Mais cette séparation des faisceaux longitudinaux n'existe pas dans le renflement moteur; ces faisceaux fictifs sont intimement unis et forment un cylindre creux composé de deux couches. La couche cylindrique de tissu fibreux est emboîtée dans la couche cylindrique de tissu cellulaire. Divisons par la pensée chacune de ces couches cylindriques en filets longitudinaux soudés 
les uns aux autres. Les filets longitudinaux du tissu cellulaire tendront tous à se courber, en dirigeant la concavité de leur courbure vers le dehors. Or, il est évident que si leur force d'incurvation est égale, la couche cylindrique qu'ils forment par leur assemblage demeurera droite et immobile. Mais si les filets longitudinaux d'un côté du cylindre creux l'emportent en force d'incurvation sur les filets du côté opposé, ceux-ci seront entraînés de force et malgré eux dans le sens de l'incurvation effectuéo par les filets qui leur sont antagonistes. Le mêrne raisonnement peut être fait par rapport à la couche cylindrique de tissu fibreux, qui est située sous la couche cylindrique de tissu cellulaire. Ainsi, chacune de ces deux couches cylindriques de tissus incurvables, agira dans cette circonstance comme s'il n'existait dans chacune d'elles que le seul côté du cylindre creux dont la force d'incurvation est prédominante. Le côté opposé du cylindre dont la force antagoniste d'incurvation sera vaincue, sera courbé malgré lui en sens inverse de sa tendance naturelle à l'incurvation, et n'agira que comme modérateur de l'incurvation du côté vainqueur. Ainsi il n'y aura dans chacun des deux cylindres creux que forment les deux tissus incurvables, que le côté le plus fort qui manifestera extérieurement son action, et cela seulement par l'excès de sa force sur celle de l'autre côté du même cylindre creux dont il contrariera et domptera l'incurvation. Ainsi, les deux cylindres creux que représentent les deux couches superposées des deux tissus incurvables, agiront comme si-le côté le plus fort de chacun de ces deux cylindres creux existait seul. 
J'applique cette théorie d'abord à l'explication du mécanisme des mouvements de réveil et de sominoil dans lo renflement moteur des folioles du haricot. Le tissu cellulaire $c$ (figures 15 et 17) est lo seul agent du réveil ou de l'élévation de la foliole, et il ne peut opérer ce mouvement que par l'incurvation de la partio supérieure $s$ de cette couche cylindrique de tissu cellulaire, parce que c'est la seule partie de cette couche cylindrique qui tende à se courber de manière à diriger vers le ciel la concavité de sa courbure. Il faut donc, pour qu'elle produise l'élévation de la foliole, que son incurvation vers le ciel soit victorieuse de l'incurvation antagoniste de la partie inférieure $i$ de cette même couche cylindrique de tissu cellulaire, cette dernière tendant en effet à diriger la concavité de sa courbure vers la terre, et par conséquent à abaisser la foliole. La supériorité de force d'incurvation de la partio supérieure $s$ de la couche de tissu cellulaire, ne vient point de la supériorité de son épaisseur; car elle n'est pas plus épaisse que ne l'est la partie inférieure $i$ de cette même couche. C'est donc dans des conditions physiologiques spéciales qu'elle puise l'excès de sa force d'incurvation. Cette partie supérieure $s$ reçoit directement l'action et l'influence de la lumière à laquelle la partie inférieure $i$ est en partie soustraite par sa position. Or, ainsi que je l'ai démontré, la lumière en augmentant la respiration des parties qu'elle frappe, y augmente par cela même l'afflux de la sève lymphatique par attraction. Cet afflux de la sève lymphatique dans le tissu cellulaire, est une condition très favorable 
à l'exercice de l'endosmose, au moyen de laquelle ce tissu cellulaire acquiert l'état de turgescence qui amène son incurvation. Ainsi, chez le renflement moteur des folioles du haricot, la supériorité do force d'incurvation de la moitié longitudinale supérieure $s$ sur la moitié longitudinale inférieure $i$ de son tissu cellulaire incurvable, est puisée tout entière dans les conditions physiologiques qui lui sont spéciales et non dans une supériorité de masse. La moitié longitudinale supérieure $s$ ainsi fortifiée physiologiquement, pourrait même être un peu inférieure en masse à la moitié longitudinale inférieure $i$, et posséder encore une force d'incurvation supérieure ì la sienne. C'est ce qui a lieu, par exemple, dans le renflement moteur des folioles $d u$ robinia pseudo-acacia, ainsi que je vais le faire voir tout à l'heure.

La cause du relèvement ou du réveil de la foliole du haricot étant ainsi déterminée, je passe à l'étudo de la cause de son abaissement ou de son sommeil, qui reconnaît pour agent la couche cylindrique de tissu fibreux $f$, dont toutes les parties concentriques tendent à diriger la concavité de leur courbure vers le centre du pétiole. Cette couche cylindrique paraît moins épaisse en haut ou du côté $s$ qu'elle ne l'est en bas ou du côté $i$ du renflement moteur. En haut, elle est même très souvent interrompue. Il semblerait donc que le côté inférieur de cette couche cylindrique de tissu fibreux étant lo plus fort par sa masse, devrait vaincre l'incurvation antagoniste du côté supérieur et, par conséquent, relever la foliole, mais c'est ce qui n'a point lieu puisqu'il est 
certain que l'abaissement do la foliole ou son sommeil, est au contraire produit par l'action du tissu fibreux incurvable par oxygénation. Cette contradiction apparente entre les faits et la théorie, disparaît devant l'observation qui montre qu'il existe au centre du renflement moteur un faisceau de tissu fibreux a (figures 15 et 17 ), tout pareil à celui de la couche $f$, et dont le sens de l'incurvation doit être tel qu'il tende à diriger la concavité de sa courbure vers lo bas, ou vers le côté $i$ du renffement moteur, ainsi que je l'ai exposé plus haut. Ainsi, ce faisceau central de tissu fibreux tend à se courber dans le même sens que lo côté supérieur de la couche cylindrique $f$ du même tissu, e'est-à-dire qu'il tend de même à abaisser la foliole ou à la mettre dans sa position de sommeil. La réunion de l'action de ces deux masses de tissu fibreux, doit nécessairement êtro victoricuse de l'incurvation antagoniste du côté inférieur de la couche cylindrique de ce même tissu fibreux, et l'entraîner de force dans le sens de la courbure vers le bas qui leur est propre. C'est ainsi que la totalité do la masse du tissu fibreux incurvable par oxygénation, produira l'abaissement ou le sommeil de la foliole par la réunion de ses incurvations congénères, lesquelles deviennent victorieuses de celles de ses incurvations qui s'y opposent.

Les folioles de la feuille du robinza pseudo-acacia offrent, comme celles de la feuille du haricot, leur réveil en se relevant et leur sommeil en s'abaissant. La structure intérieure des renflements moteurs des folioles chez ces deux végétaux est la même; elle 
sera représentée assez exactenient par les mêmes figures 15 et 17 . Cependant, chez le renflement moteur de la foliole du robinia, il y a cette différence que chez lui la coucho du tissu fibreux $f$ offre toujours une interruption complète au point $g$; en outre la couche cylindrique du tissu cellulaire $c$, est toujours plus épaissa à son côté inférieur i, qu'à son côté supérieur $s$, tandis que chez le renflement moteur do la foliole du haricot, ces deux côtés sont ordinairement égaux en épaisseur. D'après cette supériorité légère de masse, le côté inférieur $i$ de la couche de tissu celiulaire qui tend à diriger la concavité cie sa courbure vers le dehors qui est ici le bas et qui, sous ce point de vue, est antagoniste du côté supérieur $s$ qui tend de même à se courber vers le dehors qui est ici le haut, ce côté inférieur, dis-je, par la supériorité d'action, qu'il aurait par la supériorité do sa masse, devrait opérer l'abaissement de la foliole ou son sommeil; or, c'est le contraire qui a lieu puisqu'il est démontré que le tissu cellulaire c, par la généralité de son action, est l'agent du redressement de la foliole ou de srn réveil. C'est ici que l'on roit clairement l'influence qu'exerce la lumière sur la force de l'incurvation du tissu cellulaire; sous cette influence, la sève lymphatique afflue spécialement dans lo côté supérieur $s$ du renflement moteur, côté qui est frappé directement par la lumiere; le tissu cellulaire $c$ devient alors, dans cet cndroit, plus turgescent par endosmose que ne l'est la partie de ce même tissu cellulaire, qui est situéo au côté inférieur $i$; il résulte do là quo le côté supérieur $s$ du cylindro creux 
cellulaire, dirigeant la concavité de sa courbure vers le ciel avec une force prédominante, malgré l'infériorité légère de sa masse, redresse d'autant plus la foliole qu'il y a plus d'intensité de lumière; le côté inférieur $i$, du cylindre creux cellulaire qui tend à diriger la concavité de sa courbure vers la terre, est alors vaincu malgré la supériorité do sa masse et il subit de force une courbure dans un sens opposé à celui de sa tendance naturelle à l'incurvation. Voilà ce qui a lieu lorsque la feuille est placée dans l'air; les choses se passent différemment lorsqu'elle est plongée dans l'eau : alor's toutes les parties du tissu cellulaire des renflements moteurs de ses folioles, sont également pénétrées par l'eau ambianto; l'endosmose implétivo et la turgescence qui on est la suite, sont égales partout; il n'y a done plus que la supériorité de masse pour déterminer ici une supériorité de force d'incurvation dans un des côtés du cylindre creux cellulaire. Or, dans le renflement moteur de la foliole du robinia pseudoacacia, le côté inférieur de ce cylindre creux cellulaire est un peu plus épais que le côté supérieur; cette supériorité de masse lui donne donc une supériorité de force d'incurvation, lorsque la turgescence cellulaire est égale partout, ainsi que cela a lieu lors de l'inmersion de la feuille dans l'eau; c'est effectivement ce que l'expérience démontre.

J'ai dit plus haut que cette feuille, plongée dans l'eau, offre un sommeil aussi profond que dans l'air; ses folioles ont leur pointe dirigée vers la terre; or, dans le maximum de leur réveil dans l'eau, non seulement elles ne se relèvent pas vers le 
ciel, comme cela a lieu dans l'ail', mais elles n'atteignent pas même la position horizontale qui, dans l'air, est leur réveil moyen. Le maximum de leur réveil, dans l'eau, est la position intermédiaire au sommeil profond et au réveil horizontal, en sorte que le réveil de ces folioles dans l'eau, n'est, pour ainsi dire, que le quasi-révcit de ces mêmes folioles dans l'air. Dans ce maximum du réveil dans l'eau, les folioles sont inclinées obliquement vers la terre, ce qui indique que le cylindre creux de tissu cellulaire incurvable par endosmose possède plus de force d'incurvation' dans son côté inférieur que dans son côté supérieur, qui se trouve alors vaincu. Cette supériorité dé force d'incurvation du côté inférieur est ici le résultat direct et nécessaire de la supériorité de sa masse, puisque les conditions de turgescence par afflux de l'eau sont égales partout. Il est donc établi par l'observation que la supériorité de force d'incurvation de l'un des côtés du cylindre creux cellulaire, agent du réveil des feuilles, dépend tantôt de l'afflux plus grand de la sève lymphatique dans le côté vainqueur par l'influenoe de la lumière, tantôt de la supériorité de masse de meêne côté vainqueur. Ces considérations vont servir à expliquer lo nécanisme du réveil dans les renflements moteurs des pétioles de la sensitive et de 1'hedysarum strobiliferum. Chez la première de ces plantes, le réveil du pétiole a lieu par élévation, chez la secondo il a lieu par abaissement. J'ai exposé plus haut la structure intérieure du renflement moteur du pétiole de la sensitive (fig. 16 et 19). 
On a pu voir que, dans la couche cylindrique de tissu cellulaire $c$, les cellules décroissent généralement de grandeur du dehors vers le dedans; il n'y a que les cellules les plus superficielles, formant une couche extrêmement mince, qui décroissent do grandeur du dedans vers le dehors. C'est, comme on le voit, l'inverse de ce qui a licu dans le renflement moteur' des folioles du haricot, chez lequel la couche do ollules décroissantes de grandeur du dedans vers le dehors est celle qui est prédominante. Ainsi lo sens de l'incurvation du tissu cellulaire, dans lo renflement moteur du pétiole do la sensitive, est inverse de celui qui est propre à ce même tissu cellulaire dans le renflement moteur des folioles du haricot. Chez ce dernier, le tissu cellulaire se courbo en dirigeant sa concavité vers le dehors; chez la sensitive il se courbe en dirigeant sa concavité vers le dedans ou vers le centre du renflement moteur. Il résulte de là que le réveil qui, chez le pétiole do la scnsitive, a lieu par élévation comme chez la foliole du haricot, ne doit point être opéré par le même côté du cylindre creux cellulaire, qui est l'unique agent du réveil. Chez le haricot, c'est le côté supérieur $s$ (figure 15) qui, par son incurvation vers le dehors, physiologiuuement prédominante, relève la foliole et lui donne la position de réveit; chez la sensitive, c'est le côté inférieux $i$ (fig. 15) qui, par son incurvation vers le dedans, matériellement prédominante, relève lo pétiole et lui donne ainsi la position de réveil. On voit, en effet, que la couche eylindrique du tissu cellulaire est, chez la sensitive, plus épaisse à son côté inférieur $i$ qu’à 
son côté supérieur s. 'J'ai vu que le rapport de l'épaisseur relative de ces dcux côtés est à pcu près celui de 5 à 3 ; ainsi la force d'incurvation en dedans du côté inférieur $i$ du cylindre creux cellulaire, en surmontant la force d'incurvation également en dedans, et par conséquent antagoniste du côté supérieur $s$, agit comme s'il existait seul; il redresse le pétiole ou lui donne la pasition de réveil. L'abaissement du pétiole ou sa position de sommeil est le résultat de l'action du côté supérieur de l'ellipsoïdo creux fibreux $f$, aidé dans cette action par l'incurvation congénère $d u$ faisceau central de tissu fibreux $a$, de la même manière que je l'ai exposé plus haut pour le haricot. Le côté inférieur de l'ellipsoïde creux, que représente ici le tissu fibreux $f$, est alors vaincu et courbé de force dans un sens contraire à celui de son incurvation naturello. D'après cetto théorie, il est évident que, si lo faisceau central de tissu fibreux a n'existait pas pour aider le côté supérieur du tisșu fibreux à vaincre le côté inférieur, ce dernier étant ordinairoment plus épais que le côté supérieur, et par conséquent plus fort, il deviendrait vainqueur et il produirait le sommeil en élevant le pétiole; c'est effectivement ce qui a lieu dans le renflement moteur du pétiole chez l'hedysarum strobilifolium L., renflement dont la fig. 21 représente la coupe transversale. Lo tissu cellulaire $c$ tend à se courber en dirigeant sa concavité vers le dehors, lorsqu'il est rendu turgescent par l'endosmose; $f$, couche cylindrique et mince de tissu fibreux incurvable par oxygénation, et tendant à se courber vers le centre 
du renflement moteur; $d$, tissu ligneux central rayonné mêlé d'une grande quantité de gros tubes pneumatiques. Le tissu cellulaire $c$ est deux fois plus épais en bas qu'en haut; c'est donc son côté inférieur qui doit l'emporter en force d'incurvation; et comme il tend à diriger la concavité de sa courbure vers le dehor's, il doit abaisser le pétiole dans le réveil, dont le tissu cellulaire $c$ est l'agent; c'est aussi ce qui a lieu (fig. 20, b). Dans le sommeil, le pétiole se relève (fig. 20,a), et cette action de redressement est opérée par le cylindre creux de tissu fibreux $f$ (fig. 20) dont le côté supérieur est vaincu par l'incurvation plus forte du côté inférieur. Le redressement du pétiole dans le sommeil, coïncidant ici avec l'absence du faisceau central de tissu fibreux incurvable qui existe chez toutes les feuilles dont le sommeil est dans l'abaissenient, cela confirme ce que j'ai établi plus haut touchant le sens de l'incurvation de ce faisceau central de tissu fibreux $a$ (fig. 15 et 16) lequel tend à abaisser le pétiole, secondant ainsi l'action du côté supérieur de la couche cylind:ique de tissu fibreux, en sorte que le sommeil a toujours lieu dans le sens de l'abaissement, lorsque co faisceau central de tissu fibreux incurvable existe; c'est ce qui a lieu dans les renflements moteurs des folioles de haricot et de robinia pseudoacacia et du pétiole de la feuille de sensitive. Chez cette dernière plante, les folioles s'élèvent dans le sommeil comme le pétiole de l'hedysarum strobitiferum; leurs renflements moteurs ont très probablement la même organisation.

D'après ce qui vient d'être exposé, on voit que 
le réveil et le sommeil des feuilles ont lieu, chacun à part, tantôt dans la position redressée, tantôt dans la position abaissée, et l'on a vu par quel mécanisme cela s'opère. Or, il est un autre mouvement que les feuilles présentent dans leur réveil ou dans leur sommeil, c'est celui de la torsion de leur renflement moteur, torsion qui, du reste, n'existe jamais seule, mais est toujours accompagnée de l'incurvation. Ainsi, par exemple, les folioles de la feuille de la réglisse (glycyrrhiza glabra) en s'abaissant pour le sommeil, se tordent en même temps sur leur pétiole, constitué en entier par le renflement moteur, et cela 'de manière à diriger leurs faces supérieures vers l'extrémité de la feuille. Si ce double mouvement d'incurvation et de torsion du renflement moteur était plus étendu, il amènerait les deux folioles opposées à diriger leur pointe vers la terre et à se joindre, dans cette position, par leurs faces supérieures, ainsi que cela a lieu chez les feuilles des casses, dans leur position de sommeil. Chez la réglisse, la structure intérieure du renflement moteur des folioles, ne paraît pas différer sensiblement de celle que présente le renflement moteur des folioles du haricot (fig. 15 et 17), et surtout celui des folioles du robinia pseudoacacia. Ainsi, jo ne puis expliquer que rationnellement le mouvement de torsion qui lui est particulier, et qui a lieu d'une manière bien plus étendue chez les casses. Ce mouvement de torsion peut trouver l'explication de son mécanisme dans la considération de l'inégal raccourcissement longitudinal des deux tissus incurvables dont se com- 
pose leur renflement moteur. En effet, le tissu cellulaire $c$ (figure 17) possédant pendant le jour une étendue en longueur égale à celle du tissu fibreux $f$, deviendra légèrement inférieur en longueur à ce dernier pendant la nuit; car pendant le jour le tissu cellulaire $c$ est turgescent, et cessant de l'être autant pendant la nuit, ses séries de cellules alignées et contiguës qui sont moins remplies, occupent moins de longueur. Or, il doit résulter nécessairement de l'inégalité de longueur qui se sera établie le soir entre les deux couches cylindriques cellulaire et fibreuse, que celle qui sera demeurée la plus longue, c'est-à-dire la couche cylindrique fibreuse disposera ses fibres longitudinales en spirale, c'est-à-dire se tordra sur elle-même et entraînera ainsi tout le renflement moteur dans ce mouvement de torsion. C'est là le mécanisme général que j'ai assigné aux mouvements de torsion dans un autre Mémoire ${ }^{(1)}$. Le sens de cette torsion dépendra de certaines particularités d'organisation du renflement moteur qui l'exécute. D'après cette explication, il semblerait que chez les renflements moteurs des feuilles, la torsion devrait toujours accompagner l'incurvation de sommeil; or, au contraire, cette torsion dans le sommeil est assez rare. Son absence si fréquente provient, je pense, de ce que les renflements moteurs des feuilles, possèdent presque toujours au-dessous de leur couche fibreuse incurvable un tissu ligneux qui a une certaine rigidité, en sorte que s'il se prête à subir une flexion il résiste à subir une torsion,

(1) Voyez dans le Mémoire IX. [Coup-d'ail général sur les mourements des végétaux, p. 20.] 
ce dernier mouvement étant beaucoup plus difficile à imprimer. Les renflements moteurs des folioles de la réglisse et des casses auraient cela de particulier, que leur tissu ligneux serait peu rigide et se prêterait ainsi avec facilité au mouvement de torsion.

En général, chez les feuilles, lo réveil est la conséquence de l'augmentation de la lumière et par conséquent de l'augmentation de la respiration végétale, puisque celle-ci s'opère par l'assimilation de l'oxygène produit dans le tissu végétal sous l'influence de la lumière et versé dans les organes pneumatiques de la plante. J'ai fait voir dans un autre Mémoire ${ }^{(1)}$ que l'ascension de la sève par attraction, cesse d'avoir lieu lorsque la respiration de la plante est supprimée, et j'en ai conclu que c'est sous l'influence des phénomènes chimiques qui se passent dans l'assimilation de l'oxygène, que se développe la force qui attire la sève. On conçoit d'après cela, pourquoi chez les feuilles, le réveil a toujours lieu pendant le jour; car c'est alors seulement qu'elles fabriquent l'oxygène qui sert à leur respiration; c'est alors par conséquent qu'elles attirent la sève avec le plus d'abondance, ce qui est la condition la plus favorable pour la turgescence et pour l'incurvation du tissu cellulaire qui est l'agent du réveil. Lorsque le soir arrive, la diminution de la lumière en occasionnant la diminution de la respiration dans les feuilles, y produit par cela même la diminution de l'afflux de la sève attirée, et alors le tissu cellulaire agent du réveil perd une partie de sa turgescence

(1) Voyez dans le Mémoire VIII, p. 413. [Recherches sur les conduits de la sève et sur les causes de sa progression, in Mémoires, t. I.] 
et par conséquent perd une partie de sa force d'incurvation. Or, pendant la durée du jour sous l'influence d'une respiration active, le tissu fibreux agent du sommeil a augmenté peu à peu son oxygénation, laquelle se trouvant ainsi très considérable lo soir détermino l'incurvation de ce tissu fibreux, lequel l'emporte alors facilement sur l'incurvation affaiblie du tissu cellulaire agent du réveil. Alors les feuilles prennent l'état do sommeil. Cependant, dans lo courant do la nuit et dans l'absence de la respiration active qu'occasionne la lumière, le tissu fibreux, agent du sommeil, perd une partio de l'oxygène qui avait été fixé dans son tissu pendant lo jour; j'ai prouvé co fait pour les fleurs, il est par conséquent prouvé aussi pour les feuilles. Lorsque le matin arrive, le tissu fibreux, agent du sommeil, se trouve donc affaibli, le tissu cellulaire, agent du réveil, reprend de la turgescence et par suite de la force d'incurvation sous l'influence de la lumière, et le réveil des feuilles a lieu. Ainsi s'établit et so continue, pendant toute la durée de la vie des fouilles, cette oscillation diurnale qui résulto de l'action alternativement prédominante d'un tissu cellulaire incurvable par turgescence d'eau ou par endosmose implétive et d'un tissu fibreux incurvable par oxygénation. Ici doit se trouver naturellement l'exposé de ces expériences si intéressantes, faites par M. de Candolle, sur les effets que la lumière artificielle continue produit sur les feuilles susceptibles de sommeil. Ce célèbre botaniste a vu qu'en soumettant une sensitive à la lumière continue des lampes, la succession du réveil et du sommeil 
des feuilles continuait d'avoir lieu, et, ce qui est un fait bien important, que la durée de l'intervalle de temps qui séparait ces alternatives do réveil et de sommeil diminuait dans la proportion d'environ deux houres par jour. Cela permet de penser que si la sansitive, au lieu d'être soumise à la lumière artificielle continue, êtt pu so trouver soumise à la lumière continus et bien plus énergique du soleil, ello oût raccourci encore davantage la période de ses oscillations, lesquelles eussent été perpétuelles, malgré l'absence des alternatives de lumière et d'obscurité. M. de Candolle considère la continuation des mourements de réveil et de sommeil sous l'influence d'une lumière continue, comme un effet de l'habitude précédemment acquise. Ce mot habitude, auquel ne se trouve attachée aucune idée exacte, n'est véritablement qu'un voile mis à l'ignorance où nous sommes des causes auxquelles sont dus certains phénomènes vitaux qui so reproduisent quelquefois périodiquement. Ce curieux phénomène de la continuation des alternatives du réveil et $d u$ sommeil chez la sensitive, soumise à une lumière continue, me paraît devoir être envisagé d'une autre maniere : je l'exposerai dans le $\mathrm{XI}^{\ominus}$ Mémoire.

M. de Candollo a également recherché quels seraient les effets d'une obscurité continue sur le réveil et le sommeil des feuilles de la sensitive, mais il convient quo dans cette expérience.il n'a observé quo des phénomènes sans régularité. J'ai répété plusieurs fois cette même expérience, et voici ce que j'ai vu. Une sensitive placée dans des conditions favorables de température, étant mise dans une obscurité com- 
plète au moyen d'un récipient opaque qui la couvre, ses feuilles prennent d'abord la position de sommeil qu'elles conservent jusqu'au lendemain si l'expérience a été commencée dans la soirée, mais qu'elles ne conservent que jusque vers la fin du jour si l'expérience a été commencée le matin. Dans l'un et dans l'autre cas, les feuilies se déploient complètement malgré l'absence de la lumière, et elles prennent la position du réveil le plus complet. Environ dix ou douze heures après, les folioles des feuilles très jeunes se ploient tout à fait, mais leur pétiole demeure redressé; les feuilles les plus vieilles continuent à présenter le réveil le plus complet. Quant aux feuilles d'un âge intermédiaire, leurs folioles prennent un état de demi-plicature en conservant aussi leur pétiole dans un état de redressement qui excède celui du réveil normal. Cet état mixte de réveil et de sommeil des feuilles, état dans lequel le réveil est de beaucoup prédominant, persiste invariablement dans l'obscurité continue. Or, comme cette même position est prise par les feuilles de la sensitive dans le vide de la pompe pneumatique, cela prouve que c'est une position d'asphyxie. Les feuilles placées dans une obscurité continue, ne fabriquent plus d'oxygène pour en remplir leurs organes pneumatiques ou respiratoires; placées dans le vide, l'air respirable qui remplit ces organes leur est enlevé, en sorte que dans ces deux cas l'asphyxio do la plante est également produite, et les feuilles prennent exactement la même position qui est à peu près celle du réveil. J'ai fait voir, en effet, plus haut que, privées d'oxygène respira- 
toire, les fleur's et les feuilles prennent et conservent invariablement la position de réveil, et cela, par le fait de la paralysie du tissu fibreux incurvable par oxygénation et agent du sommeil. Ainsi, chez la sensitive, placée dans une obscurité continue, il n'y a qu'un seul réveil des feuilles premièrement placéas dans la position de sommeil, et ce réveil d'abord normal se change ensuite en un réveil modifié qui constituo la position de l'asphyxie. Ces phénonènes, d'autant plus prompts dans leur succession que la température est plus élevée, s'expliquent parfaitement par la théorie que j'ai exposée plus haut. Les feuilles de sensitive qui, mises à l'obscurité, ont pris la position de sommeil, la conservent jusqu'à ce que le tissu fibreux, agent du sommeil, ait perdu dans l'absence de la lumière et de la respiration normale une partie de son oxydation qui est la seule cause de son incurvation; ce tissu fibreux ainsi affaibli, le tissu cellulaire, son antagoniste et agent du réveil, reprend l'empire et place la fouille dans la position de réveil; or, comme la feuille privée de lumière ne produit plus d'oxygène respiratoire, le tissu fibreux incurvable par oxygénation, ne pouvant plus récupérer son moyen d'incurvation, qui est l'oxygène, se trouve aussi paralysé, et comme il est l'agent du sommeil, celui-ci ne se manifeste plus; la feuille reste dans un état qui ost à peu près celui du réveil.

Les expériences qui viennent d'être exposées prouvent que, lors do la suppression complète de l'oxygène respiratoire dans les organes pneumatiques des feuilles, l'incurvation du tissu cellulaire agent 
du réveil existe seule, et que l'incurvation du tissu fibreux, agent du sommeil, est supprimée; cela semblerait prouver que l'existence de la respiration végétale, nécessaire évidemment pour l'action du tissu fibreux incurvable par oxygénation, serait inutile pour l'action du tissu cellulaire incurvable par turgescence d'eau ou par endosmose; or cela est si loin d'être vrai que c'est le contraire qui a lieu; dans l'état naturel, l'existence de la respiration végétale normale est plus nécessaire pour l'action du tissu cellulaire aggent du réveil, que pour l'action $d u$ tissu fibreux agent du sommeil. Cette assertion, qui paraît paradoxale au premier coup-d'œil, est prouvée par les expériences suivantes.

Je pris trois feuilles de haricot que je nommerai A, B, C. La feuille A fut submergée et mise pendant un quart d'heure dans le vide : en lui rendant l'air, les cavités pneumatiques furent entièrement remplies d'eau. La feuille $B$ resta aussi pendant un quart d'heure dans le vide, mais sans submersion. La feuille C demeura dans l'état naturel. Je mis ces trois feuilles tremper par leur pétiole dans des vases remplis d'eau, que je plaçai dans un lieu bien éclairé par la seule lumière diffuse. Lorsque le soir arriva, la feuille A présenta la première le phénomène de l'abaissement de ses folioles ou du sommeil; la feuillo B présenta plus tard ce phénomène, lequel arriva encore plus tard chez la feuille C. Le lendemain, la feuille C présenta la première lo phénomène du redressement de ses folioles ou du réveil. La feuille $B$ se réveilla plus tard, et enfin la feuille A se réveilla la der- 
nière; mais le réveil de ces deux dernières feuilles fut incomplet; leurs folioles restèrent pendant toute la journée dans un état de demi-sommeil, et elles ne firent aucun mouvement de nutation pour se diriger vers la lumière. La feuille $\mathrm{C}$, au contraire, non seulement redressa complètement ses folioles, ce qui constitue l'acte de leur réveil, mais elle inclina leur face supérieure vers la fenêtre de laquelle venait la lumière, ce qui constitue l'acte de leur nutation. Le soir de ce second jour la feuille A commença encore la première à présenter le phénomène du somnieil; elle fut suivie par la feuille $B$ et enfin par la feuille C. Celle-ci cessa en même temps de tenir la face supérieure de ses folioles inclinće vers la fenêtre; la position de nutation cessa d'avoir lieu pendant la nuit, et les folioles reprirent leur position naturelle. Le troisième jour la feuille $\mathrm{A}$ ne présenta point le phénomène du réveil; elle commença à se faner. La feuille $B$ se réveilla un'peu, mais elle était languissante. La feuille C, parfaitement vivante, exécutait ses fonctions comme à l'ordinaire. Le quatrième jour la feuille A était morte; la feuille B commença à se faner et fut morte lo lendemain. La feuille $\mathrm{C}$ continua longtemps à vivre.

On voit par ces expériences que la feuille A, dont les organes pneumatiques avaient été vidés d'air et remplis d'eau en grande partie, fut plus hâtive pour le sommeil et plus tardive pour le réveil que ne le fut la feuille B, dont les organes pneumatiques vides d'air étaient cependant restés en partie accessibles à son retour; je dis en partie, car il est certain 
que lors de la soustraction par la pompe pneumatique de l'air contenu dans les canaux pneumatiques d'une plante, os canaux doivent être envahis en partie par les liquides séveux. Les deux feuilles A et B ne présentèrent point de nutation comme la feuille $\mathrm{C}$ qui avait conservé ses organes pnemmatiques dans leur état naturel; cette feuille $\mathrm{C}$ fut en outre plus tardive pour le sommeil, et plus hâtive pour le réveil que ne lo furent les deux feuilles $\mathrm{A}$ et $\mathrm{B}$. Ainsi le réveil des feuilles est plus altéré que leur sommeil par la diminution de la respiration végétale, alors leur réveil est plus court et leur sommeil plus long que dans l'état naturel. Ces faits s'expliquent facilement par les considérations suivantes.

Le tissu cellulaire incurvable par endosmose, et agent du réveil, ne peut évidemment devenir turgescent et par suite se courber que lorsque la sève lymphatique lui est apportée en quantité suffisante. Or, j'ai démontré que l'ascension de la sève lymphatique par attraction cesse d'avoir lieu lorsqu'il n'y a plus d'oxygène respiratoire dans les organes pneumatiques des plantes, et que cette ascension est proportionnelle en général à la quantité de la respiration végétale; or, les trois feuilles de haricot, sujets des dernières expériences, avaient évidemment des quantités différentes de respiration. La feuille A était celle qui respirait le moins; la feuille B respirait un peu plus, mais toujours d'une manière insuffisante. Chez ces deux feuilles, l'ascension do la sève lymphatique par attraction était done faible, et coḿme elle devait compenser la perte faite par la 
transpiration des feuilles, il n'arrivait dans leur tissu assez de sève lymphatique pour produire la turgescence du tissu cellulaire, agent du réveil, que lorsque la lumière devenue intense augmentait à la fois la respiration végétale et l'ascension de la sève lymphatique, en sorte que le réveil arrivait tard. Le soir dès que la lumière commençait à diminuer, la respiration et l'ascension de la sève lymphatique diminuaient en même temps, et d'une manière considérablo en raison de leur faiblesso antécédente, en sorte que le tissu cellulaire agent du réveil, n'ayant plus assez d'eau pour conserver la turgescence par endosmose qui est la cause de son incurvation, cessait alors d'être plus fort que le tissu fibreux dont l'incurvation antagoniste, devenue victorieuse, produisait ainsi le sommeil qui arrivait de bonne heure et plus tôt que dans l'état naturel. Ainsi, d'après ces observations, il faut plus de respiration végétale, il faut plus d'oxygène dans les organes pneumatiques des feuilles, pour déterminer une ascension de sève lymphatique suffisante pour produire la turgescence et l'incurvation du tissu cellulaire agent du réveil, que pour produire l'incurvation par oxygénation du tissu fibreux agent du sommeil, en sorte que lors de la diminution de la respiration végétale, le tissu cellulaire agent du réveil est plus altéré dans l'exercice de sa fonction d'incurvation que ne l'est le tissu fibreux agent du sommeil. Cela n'a lieu cependant que lorsque les feuilles sont placées à l'air libre et à la lumière, c'est-à-dire, lorsqu'elles sont dans la position qui favorise lo plus leur transpiration. En effet, j'ai fait voir que lorsque les 
feuilles sont placées à l'obscurité, laquelle diminue la transpiration végétale, ou bien lorsqu'elles sont plongées dans l'eau non aérée où cette transpiration est nulle et où il y a au contraire absorption immédiate de sève lymphatique; ou bien enfin lorsqu'elles sont placées dans le vide de la pompe pneumatique, où la perte d'eau par l'évaporation est également à peu près nulle, l'abolition de la respiration qui a lieu dans ces trois circonstances, diminue à peine ou ne diminue point du tout l'action du tissu cellulaire agent du réveil, tandis qu'elle abolit l'action du tissu fibreux agent du sommeil. Cela provient de co que dans ces trois circonstances il y a dans les feuilles assez de sève lymphatique, pour produire la turgescence et l'incurvation du tissu cellulairo agent du réveil; il n'est donc pas nécessaire que la respiration végétale existe pour déterminer l'ascension de cette sève lymphatique, comme cela a lieu lorsqu'il s'agit de remplacer celle que l'évaporation soustrait, ce qui abolit la turgescence cellulaire.

Il résulte de ces considérations et des expériences qui y ont donné lieu, que le maximum de l'action vitale chez les feuilles a lieu pendant le jour ou dans leur réveil, et que leur sommeil qui a toujours lieu pendant la nuit coïncido avec une diminution de cette même action vitale qui est en proportion, chez tous les êtres vivants, avec la quantité de la respiration. Ces considérations tendent à établir une véritable similitude entre le sommeil des végétaux et celui des animaux, similitude que l'on était loin de soupçonner, car généralement on considère 
comme métaphoriques les expressions de sommeil et de réveil appliqués aux végétaux. Il est bien entendu que, dans ce rapprochement, je ne prétends point comprendre les phénomènes du sommeil sensorial propre aux seuls animaux; je ne considère ici le sommeil chez les végétaux, que dans ses phénomènes purement organiques, lesquels attestent tous une diminution de l'action vitale, diminution qui est le seul point de rapprochement que je prétende établir entre le sommeil des animaux et celui des végétaux. C'est par le fait de cette diminution de l'action vitale que chez les parties des végétaux qui sont susceptibles de sommeil et de réveil, la mort est toujours précédé par le sommeil, ainsi que cela a lieu chez les animaux. C'est dans la position de sommeil que les fleurs et les feuilles meurent ordinairement, en sorte que chez les végétaux, comme chez les animaux, le sommeil est l'image de la mort.

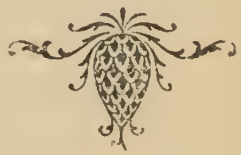




\section{Table des Matières.}

Page.

Notice biographique ......................................... vir

Coup-d'œil général sur les mouvements des vágétaux........ 1

Du réveil et du sommeil des plantes.......................... 35 



University of British Columbia Library

DUE DATE

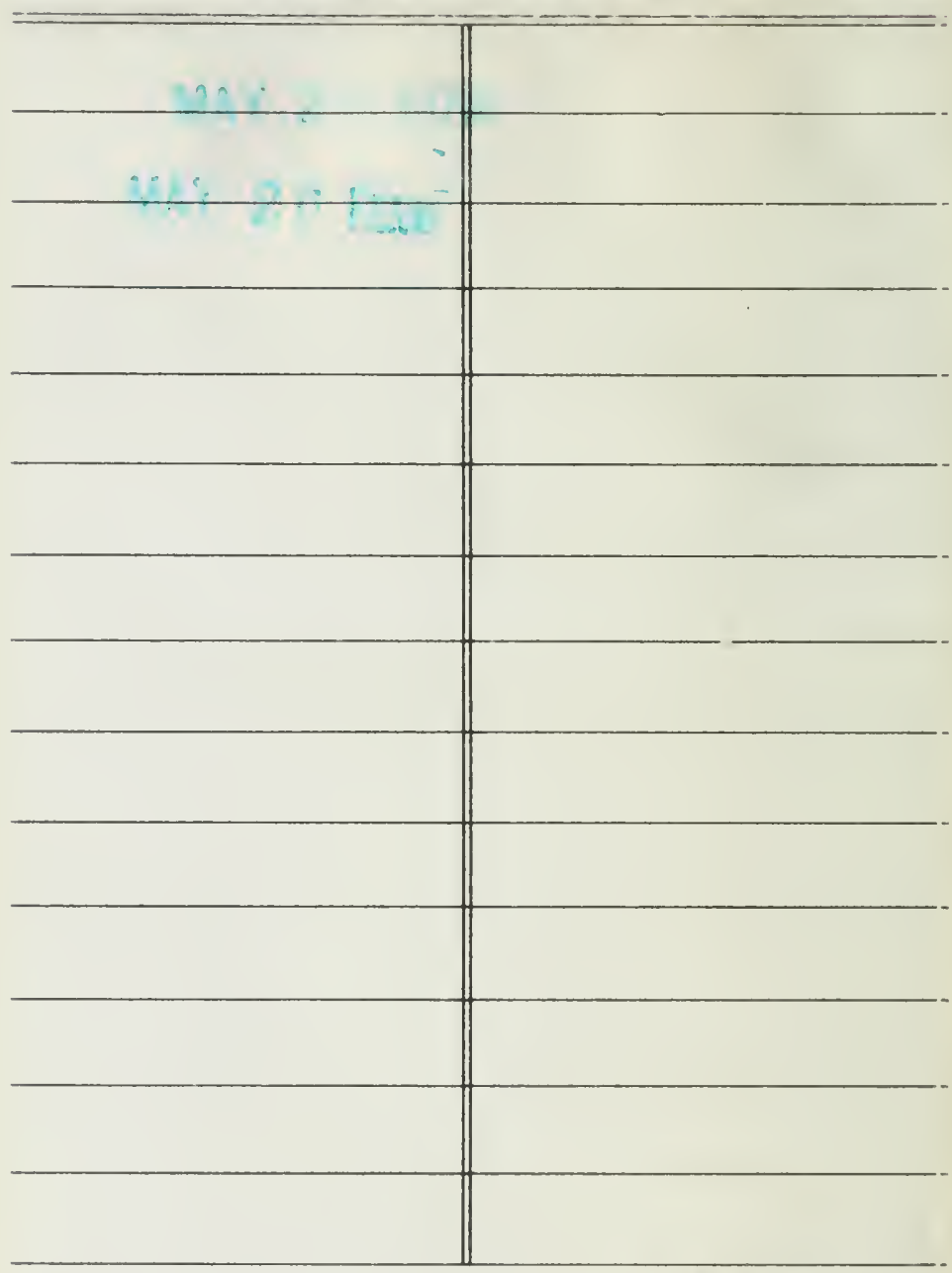

ET -6 

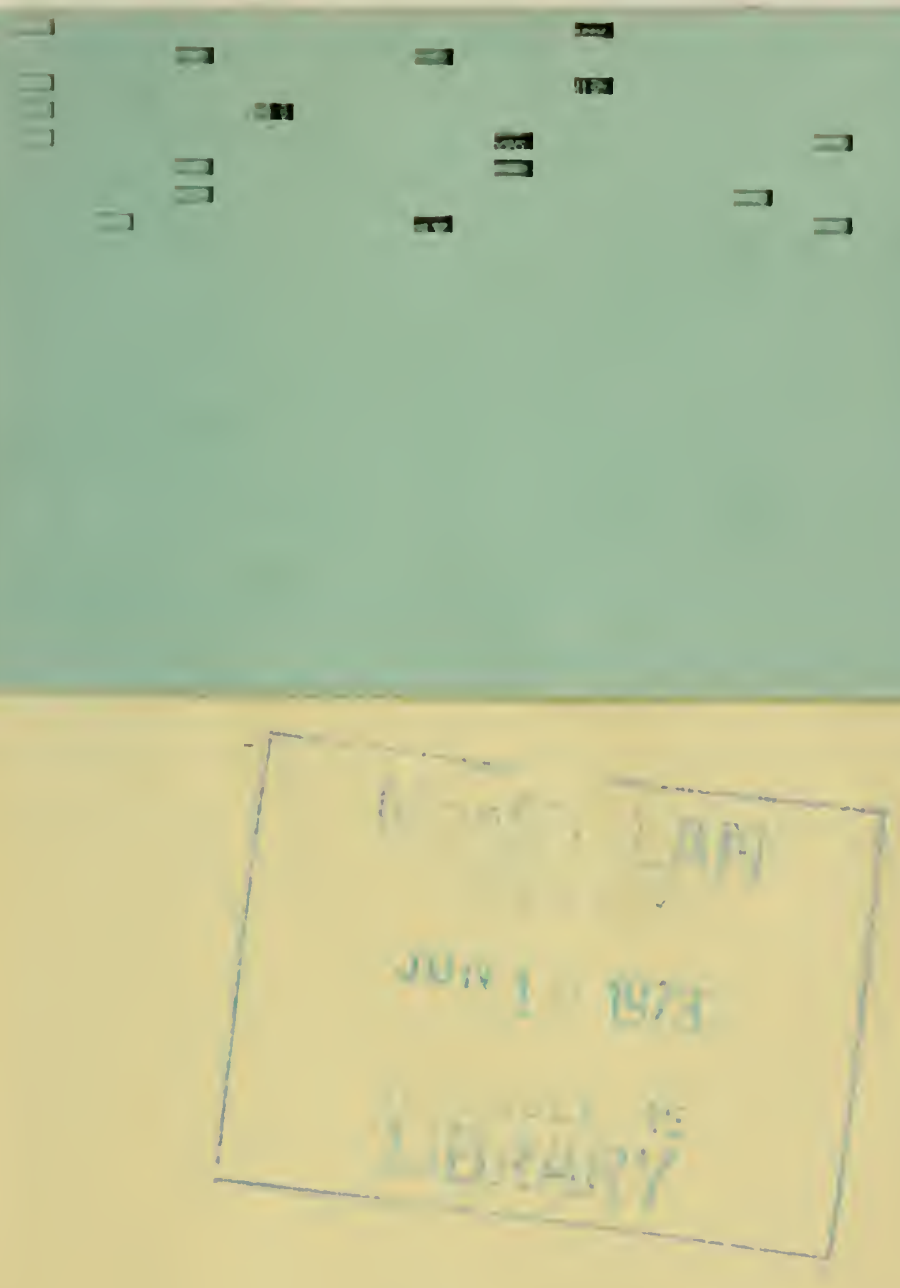
